\title{
Reciprocity in Dynamic Employment Relationships
}

by

Matthias FAHN

Working Paper No. 2012

June 2020

Johannes Kepler University of Linz Department of Economics Altenberger Strasse 69 A-4040 Linz - Auhof, Austria www.econ.jku.at 


\title{
Reciprocity in Dynamic Employment Relationships*
}

\author{
Matthias Fahn ${ }^{\dagger}$
}

June 30, 2020

\begin{abstract}
This paper analyzes a dynamic relational contract for employees with reciprocal preferences. I develop a tractable model to investigate how "direct" performance-pay (promising a bonus in exchange for effort) and generous upfront wages (which activate the norm of reciprocity) interact over the course of an employee's career. I show that firms can benefit from committing to paying non-discretionary wages in the future as this boosts their credibibility in the relational contract. The reason is that these wages have to be paid under any circumstances, whereas employees only reciprocate if the firm has kept its promises. Moreover, I demonstrate that more intense competition for workers can intensify the use of reciprocity-based incentives.
\end{abstract}

Keywords: reciprocity, relational contracts, commitment, norms and social preferences JEL Classification Numbers: C73, D21, D86, D90, D91

\footnotetext{
${ }^{*}$ I am grateful to Daniel Barron, Florian Ederer, Florian Englmaier, Willie Fuchs, Bob Gibbons, Marina Halac, Paul Heidhues, Fabian Herweg, Johannes Hörner, Hideshi Itoh, Nicolas Klein, David Miller, Takeshi Murooka, Aniko Öry, Anna Sanktjohanser, Johannes Schneider, Heiner Schumacher, Peter Schwardmann, Dirk Sliwka, Joel Sobel, Marta Troya Martinez, Joel Watson, Nick Vikander, Rudi Winter-Ebmer, Giorgio Zanarone, and Christian Zehnder, as well as seminar participants at Graz, Copenhagen, Linz, Madrid (Carlos III), UC San Diego, University of Montreal, Munich, Vienna, the 2018 Bavarian Micro Workshop (Regensburg), the 2019 Osaka Workshop on Behavioral Contract Theory, the 2019 SIOE conference (Stockholm), the 2019 German Economic Association Conference, the 2020 Conference on Mechanism and Institution Design (online), and the 2020 Workshop on Relational Contracts (online), for their valuable comments and suggestions. Financial support by the Hardegg'sche Stiftung, by Deutsche Forschungsgemeinschaft through CRC TRR 190, and by the FWF through project $P$ 33307-G is gratefully acknowledged.

$\dagger$ JKU Linz and CESifo; matthias.fahn@jku.at.
} 


\section{Introduction}

Motivating their employees is of utmost importance for firms. A well-designed incentive scheme can increase profitability, yet requires a link between employees' payoffs and their performance (Prendergast, 1999). However, although many employees receive some form of performance pay, most real-world labor contracts specify significant salaries which are recurring and non-discretionary. ${ }^{1}$ Such an inflexible commitment to wage payments seems detrimental to the provision of incentives, in particular if legal institutions - such as employment protection laws - or contractual arrangements limit the use of firing threats.

In this paper, I show how the commitment to pay non-discretionary wages can facilitate the provision of incentives by increasing a firm's credibility when making informal promises. For that purpose, I assume that formal, court-enforceable, incentive contracts are not possible, and informal relational contracts must be used to motivate employees. ${ }^{2}$ Moreover, I take into account that such a relational contract might not only determine an employee's performance-based pay, but also establish a social norm to reciprocate generous wages. Importantly, this norm only induces reciprocal behavior if the firm has honored the informal agreement so far. Then, guaranteeing non-discretionary wages in the future allows the firm to credibly promise a higher performance-based bonus today. The reason is that such wages have to be paid irrespective of the firm's previous behavior, whereas employees only reciprocate by exerting higher effort if earlier-made promises have been kept.

The idea that a relational contract can establish a norm to reciprocate goes back to Macneil (1980; 1983) who developed a norms-based approach to contracting, in which a relational contract is a manifestation of the norms supposed to govern the behavior of the involved parties This perception has been supported by recent evidence from Kessler and Leider (2012), Krupka et al. (2017) and MacLeod et al. (2020) who demonstrate that contracts - in particular informal "handshake agreements" - can generate inherent enforcement mechanisms by establishing norms that parties feel obliged to honor. Moreover, norms have been found to respond to circumstances. For example, Kimbrough and Vostroknutov (2016) state that a small change in context can substantially alter the norms governing a situation, which consequently influences the extent of the

\footnotetext{
${ }^{1}$ Indeed, there is evidence that even though the compensation of most employees is somehow tied to their performance, a substantial share of their salary is not (Lemieux et al., 2009, p. 22). Also see Bloom and Reenen (2011) for a survey on the prevalence of incentive pay.

${ }^{2}$ Those are self-enforcing agreements based on observable but non-verifiable information which are used if individual contribution to firm value cannot be measured objectively; see Prendergast (1999), Kampkötter and Sliwka (2016), or Frederiksen et al. (2017), for evidence on the importance of incentive schemes based on informal, "subjective" assessments of performance.
} 
prosociality of actions. Fehr and Schurtenberger (2018) demonstrate that norms pushing for prosocial behavior can quickly emerge but also decay. Landmann and Vollan (2020) show that politicians become more pro-social after being elected.

I integrate these insights into a repeated principal-agent model with a predetermined last period. The risk-neutral agent can exert costly effort which benefits the risk-neutral principal and is observable but not verifiable. I first derive a profit-maximizing arrangement without any formal commitment, hence formal, court-enforceable, contracts cannot be used to motivate the agent. Instead, both parties may form a self-enforcing relational contract which determines bonus payments the principal is supposed to make to reward the agent's effort. In addition, the relational contract specifies a norm of reciprocity, implying that a generous wage payment by the principal is supposed to be reciprocated by the agent via higher effort. The agent responds to this norm because he has preferences for reciprocity. These preferences might be individual- or matchspecific (for example because the agent has developed sentiments for the principal; see Akerlof, 1982) and are activated by non-discretionary wage components, by which I mean payments that are not paid as a reward for past effort. Thus, incentives can be provided (i) directly by promising a bonus to be made after the agent has exerted effort, and (ii) indirectly via the norm of reciprocity and paying a non-discretionary wage before the agent is exerting effort. For the former, the principal uses "relational incentives", where the bonus is enforced by repeated game incentives. For the latter, the principal uses "reciprocity-based incentives". This specification allows for a recursive structure of the optimization problem and a separation of both incentive tools (i.e., each payment is designated to exactly one of each).

The agent's responsiveness to the norm of reciprocity is affected by the history of the relationship. If the principal reneges on a promised bonus, not only does the relational contract break down, but also the agent's preferences for reciprocity toward the principal disappear. Importantly, the norm function allows the use of relational incentives even though there is a predetermined last period. Because the agent's preferences for reciprocity disappear once the principal reneges on a promised bonus and because the principal's profits in the last period of the game are higher with reciprocal preferences than without, her behavior in the penultimate period affects her profits in the last period. This interaction between relational and reciprocity-based incentives carries over to earlier periods and enables the principal to credibly promise an effort-based bonus. The maximum size of this bonus is determined by the so-called dynamic enforcement (DE) constraint, which states that a bonus must not exceed the difference between future discounted profits on and off the equilibrium path. Since future on-path profits increase in the extent of the agent's reciprocal preferences, the principal can also provide stronger 
relational incentives today if the agent is more reciprocal. This source of complementarity between relational incentives and the agent's reciprocal preferences is supported by an additional channel. The (DE) constraint in a given period is relaxed and more effort can be implemented if she pays a non-discretionary wage in this period, implying that reciprocity-based preferences are particularly valuable whenever the constraint binds. Therefore, relational and reciprocity-based incentives are complements and more effort is implemented with a combination of the two. Both are dynamic substitutes, however, in the sense that relational incentives are gradually replaced by reciprocitybased incentives over time. The reason is that the (DE) constraint is "tighter" in later periods (having fewer remaining periods reduces the principal's future profits), which amplifies the benefits of reciprocity-based incentives as time passes. This implies that a profit-maximizing incentive scheme has the highest effort in the early stages of the employment relationship, where it remains until the (DE) constraint starts to bind. Then, the principal's reduced credibility effectively constrains her ability to pay a sufficiently high bonus. This gradually decreases effort, which in turn lets the principal respond with an increase in the non-discretionary fixed wage and consequently place a higher weight on reciprocity-based incentives.

After deriving a profit-maximizing relational contract without formal commitment, I turn to the case in which the principal can commit to paying non-discretionary wages in subsequent periods (still, effort and output are non-verifiable, hence formal incentive contracts continue to be infeasible). This resembles actual labor market contracts which often have longer time horizons and specify the recurring payment of fixed or deterministically increasing salaries. Such a commitment is usually not deemed optimal for the provision of incentives because it limits the principal's options to let the agent's payoff respond to his performance (Prendergast, 1999). This holds in particular for jobs where wages cannot be regarded as a reward for employees' career concerns. Still, long-term commitment can be optimal with risk-averse agents (Harris and Holmstrom, 1982) or in search-and-matching models of the labor market (see, for example, Burdett and Mortensen, 1998 or Mortensen and Pissarides, 1999). These models rarely consider incentives for employees to exert effort, thus one might conclude that firms face a trade-off between efficiently motivating employees and effectively addressing other labor market frictions. To the contrary, I show that committing to future non-discretionary payments increases the principal's credibility in a relational contract with a reciprocal agent (in particular in later stages of a career), because it allows her to credibly promise a higher discretionary bonus. The reason is that the future wage has to be paid even if the principal has reneged on today's bonus, whereas the agent will only reciprocate to this wage if the bonus has been paid. 
Extensions In Section 5, I derive a number of extensions. First, I allow for a general reference wage in Subsection 5.1 (as opposed to the main part where I have assumed that the agent reciprocates to any positive non-discretionary wage). I argue that such a reference wage is shaped by aspects such as labor market competition or the unemployment rate. Then, more intense competition for workers can intensify the use of reciprocity-based incentives by increasing the agent's reference wage - because of a reduced quasi-rent generated in the relationship. Thus, I contribute to the discussion of how competition affects the relevance of social preferences. Whereas competition reduces the importance of social preferences if contracts are complete (Fehr and Schmidt, 1999; Dufwenberg et al., 2011), it appears to matter less if contracts are incomplete (Fehr and Fischbacher, 2002; Schmidt, 2011). I demonstrate that social preferences can even become more important in a more competitive environment because the power of relational incentives is restricted. Finally, a lower reference wage increases effort, however only with a binding (DE) constraint, i.e. not in the first and very last periods.

In Subsection 5.2, I let the agent's preferences for reciprocity not merely be triggered by non-discretionary, but by all realized payments (i.e., also by wages paid in response to past effort). Then, only upfront wages and no bonuses are used to compensate the agent. Still, the ability to commit to paying non-discretionary wages in the future relaxes her (DE) constraint.

Some additional results are generated in Subsection 5.3, where I allow for asymmetric information on the agent's reciprocal preferences (analyzed within a two-period setting). There, I assume that the agent might either be reciprocal (as in the previous analysis) or selfish (i.e., without any reciprocal preferences). If the likelihood of facing a reciprocal agent is high, a "separating contract" is optimal for the principal. This incorporates high effort in the first period which however will only be exerted by the reciprocal type, whereas the selfish type shirks and is subsequently fired. If the likelihood of facing a selfish agent is high, it might be optimal for the principal to offer a "pooling contract". This incorporates low effort in the first period which is exerted by both types. In the second period, the selfish type collects the wage and subsequently shirks. The pooling contract resembles outcomes derived in the reputation literature (see Mailath and Samuelson (2006) for an overview), in which the presence of even a small proportion of "commitment types" can motivate selfish agents to cooperate in a finitely repeated game because it allows them to maintain a reputation for (potentially) being cooperative. Furthermore, it is a common perception that in lab experiments with repeated interaction, selfish types who imitate cooperative (or "fair") types are responsible for driving high cooperation in early periods (Fehr et al., 2009).

However, the existence of the pooling contract in my setting relies on a perfect 
Bayesian equilibrium being played at which any deviation from equilibrium effort lets the principal assign probability 1 to facing the selfish type. Nonetheless, even if preferred by the principal, such a pooling contract may not satisfy the intuitive criterion (Cho and Kreps, 1987). Deviation to a higher effort than that specified by the pooling contract would only be incentive compatible for the reciprocal type, but not for the selfish type. Such an upward deviation would thus reveal the agent to be reciprocal and allow for an adjustment of the second-period wage that makes the principal and reciprocal agent better off. Then, only a separating contract can be sustained.

Further extensions are analyzed in Appendix A where I demonstrate the robustness of my results.

Evidence Besides deriving the novel result that inflexible formal payments have a positive effect on the principal's ability to use informal incentives, an optimal relational contract with a reciprocal agent can deliver explanations for a number of empirical observations.

First, Boosey and Goerg (2018) find that relational and reciprocity-based incentives indeed are complements and that a relational contract with agents who are known to be reciprocal can even be sustained with a finite time horizon. They conduct a lab experiment in which a manager and a worker interact for two periods. The worker can spend time completing a series of real effort tasks and is paid an upfront wage in every period. In addition, the principal may have the opportunity to pay a fixed bonus between the two periods, after the first period output has been observed. Boosey and Goerg (2018) find that average output is considerably larger with this option than in those treatments in which the principal either cannot pay a bonus (in which case a positive effort is still observed, indicating that the participants have reciprocal preferences), or the bonus can be paid at the beginning or end of the game.

Second, consistent with the described effort dynamics, there is evidence that a worker's productivity decreases once he approaches retirement. Using US data, Haltiwanger et al. (1999) find that a firm's productivity is higher if it has a lower proportion of workers older than 55. Skirbekk (2004) reports that older workers generally have lower productivity and are overpaid relative to their productivity. Using Belgian data, Lallemand and Rycx (2009) show that having a high share of workers above 49 is harmful for a firm's productivity. Reduced effort in the last periods of an employment relationship has also been observed in many lab experiments (e.g., Brown et al., 2004; Fehr et al., 2009). Those results have mainly been attributed to selfish individuals imitating those with social preferences in early periods to collect rents later on. This explanation can account for the observed effort dynamics, but not for the high amount of separations in 
initial periods. Therefore, the separating contract in the case of asymmetric information (Subsection 5.3) can provide a complementary interpretation of the higher cooperation in lab experiments with repeated interaction.

Third, I show that effort is higher if the agent has more pronounced preferences for reciprocity (Subsection 3.2.4), which has been observed by Dohmen et al. (2009). They use data from the German Socio-Economic Panel (SOEP), an annual panel survey representative of the German population that contains a wide range of questions on the personal and socioeconomic situation as well as labor market status and income of respondents. In a number of years $(2005,2010$, and 2015), it also contained questions designed to capture individual reciprocal inclinations. As a measure of (non-verifiable) effort, Dohmen et al. (2009) use overtime work, finding that individuals with stronger reciprocal inclinations are more likely to work overtime. Moreover, in my setting the positive effect of reciprocal preferences on effort is stronger if reciprocity-based incentives are more important (i.e., in later periods when the (DE) constraint binds; Subsection 3.2.4). This outcome is in line with evidence provided by Fahn et al. (2017). Using the same data and approach as Dohmen et al. (2009), they show that the positive interaction between reciprocal inclinations and effort is substantially stronger for older workers close to retirement.

Fourth, I argue that higher unemployment can generally increase effort because of the moderating effect on the reference wage, an outcome observed by Lazear et al. (2016). They argue that this is due to a firing threat (which also underlies efficiency wage models and there generates similar results), which however has received little support in surveys among managers (Bewley, 1999). Thus, I provide an alternative mechanism for a positive relationship between unemployment and effort which does not rely on a firing threat for non-performance. Furthermore, my model would predict that this effect is absent at the beginning and very end of a career, an aspect that to my knowledge has not been assessed so far.

Summing up, this paper makes three contributions. First, I demonstrate how nondiscretionary formal payments facilitate the use of informal performance pay. Thus, there does not need to be a discrepancy between real-world labor contracts containing fixed salaries and the optimal provision of incentives. Second, I develop a tractable framework to incorporate reciprocal preferences into a relational contracting model, which allows for a relational contract with a predefined last period. I show how relational and reciprocity-based incentives interact over the course of an employee's career and present evidence for my main results. Third, I shed new light on the consequences of labor market competition for the importance of social preferences in the workplace. 


\section{Related Literature}

One of the most robust, thoroughly researched outcomes in behavioral economics is that individuals not only maximize their own material payoffs, but also take others' well-being into account when making decisions (DellaVigna, 2009). Many individuals seem to possess social preferences, where an important component is captured by preferences for intrinsic reciprocity. Plenty of research since Fehr et al. (1993) and Fehr et al. (1998) has found experimental support for the existence of reciprocal preferences (see Camerer and Weber (2013) for an overview of experimental research, or DellaVigna and Pope (2018) and DellaVigna et al. (2019) for more recent evidence). Most of these exercises have been careful to rule out repeated interaction in order to isolate the effect of social preferences. However, to matter in the workplace, reciprocal preferences should not be marginalized by repeated game considerations. It is thus crucial to understand how repeated interaction affects the optimal provision of incentives for reciprocal individuals (Sobel, 2005). Some experimental studies have approached this question and disentangled the two motives for cooperation. Reuben and Suetens (2012) use an infinitely repeated prisoner's dilemma to assess the relative importance of strategic motives (i.e., driven by repeated interaction) and intrinsic reciprocity and find that cooperation is mostly driven by strategic concerns. Similarly, Dreber et al. (2014) find that strategic motives seem to be more important than social preferences in an infinitely repeated prisoner's dilemma. Cabral et al. (2014) conduct an infinitely repeated veto game to distinguish between explanations of generous behavior. They find strategic motives to be the predominant motivation, but also present evidence for the importance of intrinsic reciprocity. Hence, experimental evidence suggests that repeated game incentives are an important mode to support cooperation even for individuals with reciprocal preferences. However, a sound understanding of how firms optimally design dynamic incentive schemes for reciprocal agents is still lacking. The present paper addresses this gap by providing a tractable theoretical framework that incorporates the norm of reciprocity into a relational contracting framework.

The theoretical literature on intrinsic reciprocity can be arranged along the lines of whether reciprocal behavior is merely triggered by outcomes or whether the counterpart's intentions matter as well. The classic gift exchange approach developed by Akerlof (1982) is an example of outcome-based reciprocity, where firms can strategically use wages above the market-clearing level to induce their employees to work harder. Applying this idea to a moral hazard framework with reciprocal agents, Englmaier and Leider (2012a) show that generous compensation can not only be a substitute for performance-based pay, but may also increase profits. This is different from Rabin's 
(1993) assumption that the perceived kindness of an action should be the driving force to induce reciprocal behavior. Dufwenberg and Kirchsteiger (2004) apply this psychological game theory to extensive games. Segal and Sobel (2007) demonstrate how a player's preferences over strategies might be represented as a weighted average of the utility from outcomes of the individual and his opponents. Netzer and Schmutzler (2014), however, state that the extent to which intention-based reciprocity can explain gift exchange in the workplace is limited. They argue that, if only intentions matter, a self-interested firm cannot benefit from its employees' reciprocal preferences. Falk and Fischbacher (2006) develop a theory incorporating both aspects, outcomes and intentions. They assume that an action is perceived as kind if the opponent has the option to treat someone less kind. They also discuss evidence that while individuals respond to outcomes, those responses are considerably stronger if the choices are at the counterpart's discretion (see Falk et al., 2006; Fehr et al., 2009; Camerer and Weber, 2013). Cox et al. (2007) and Cox et al. (2008) develop a theoretical framework that can generate such results without having to resort to psychological game theory. Under their approach, which is based on neoclassical preference theory, individuals merely respond to observable events and opportunities instead of beliefs about others' intentions or types. I develop these ideas further and adapt them to a dynamic setting.

I also contribute to the literature on relational contracts. Bull (1987) and MacLeod and Malcomson (1989) derive relational contracts with observable effort, whereas Levin (2003) shows that those also take a rather simple form in the presence of asymmetric information. Malcomson (2013) delivers an extensive overview of the literature on relational contracts. Within this broader area, a few papers have investigated the implications of incorporating "behavioral" components into a relational contracting framework. Dur and Tichem (2015) incorporate social preferences into a model of relational contracts and show that altruism undermines the credibility of termination threats. Kragl and Schmid (2009) demonstrate that having a relational contract with inequity averse agents might reduce the principal's commitment problem, whereas Fahn and Zanarone (2020) explore how envious social comparisons among agents affect the trade-off between pay secrecy and transparency in a relational contracting setting. Fahn and Hakenes (2019) show that teams can serve as a commitment device for present-biased individuals. To the best of my knowledge, the present paper is the first to incorporate intrinsic preferences for reciprocity into a relational contracting framework, and the first to show that the norm of reciprocity allows for the formation of relational contracts even with a predefined last period. 


\section{Model Setup}

\subsection{Environment and Technology}

There is one risk-neutral principal ("she") and one risk-neutral agent ("he"). At the beginning of every period $t \in\{1, \ldots, T\}$, with $2<T<\infty$, the principal decides whether to make an employment offer to the agent or not $\left(d_{t}^{P} \in\{0,1\}\right)$. In case an offer is made $\left(d_{t}^{P}=1\right)$, it specifies an upfront wage $w_{t} \in \mathbb{R}_{+}{ }^{3}$ The agent's acceptance/rejection decision is described by $d_{t}^{A} \in\{0,1\}$. Upon acceptance $\left(d_{t}^{A}=1\right)$, the agent receives $w_{t}$ and chooses an effort level $e_{t} \in \mathbb{R}_{+}$, which is associated with effort costs $c(e)=$ $e^{3} / 3 .{ }^{4}$ Effort generates a deterministic output $e_{t} \theta$, with $\theta>0$, which is subsequently consumed by the principal. Afterwards, the principal can pay a discretionary bonus $b_{t} \in \mathbb{R}_{+}$. If the principal refrains from making an offer $\left(d_{t}^{P}=0\right)$ or if the agent rejects an offer made by the principal $\left(d_{t}^{A}=0\right)$, both consume their outside option utilities which are set to zero. Moreover, the principal and agent share a discount factor $\delta \in(0,1]$.

\subsection{Relational Contract, Preferences, and the Norm of Reciprocity}

Neither effort nor output is verifiable, however can be observed by both parties. Therefore, only relational but no formal incentive contracts are feasible. For now, I also rule out any long-term commitment; a formal commitment to paying non-discretionary wages in future periods is considered in Section 4. The relational contract is a selfenforcing agreement determined by principal and agent and constitutes a subgame perfect equilibrium of the game. In addition to the standard components of a game players, information, action spaces, preferences, and equilibrium concept - I incorporate a norm function that activates the norm of reciprocity and maps the game's history into the agent's preferences. Before introducing this norm, I formally describe histories and feasible strategies. A discussion of several assumptions made with respect to the norm of reciprocity follows in Section 2.3.

\footnotetext{
${ }^{3}$ The non-negativity constraint simplifies the definition of reciprocity below in a sense that I do not have to differentiate between positive and negative payments. Moreover, I want to rule out negative up-front wages which could allow the principal to extract "reciprocity rents" that the agent enjoys later.

${ }^{4}$ I assume this specific functional form for analytical tractability. Other (convex) cost functions deliver similar results as long as the third derivative is positive. A positive third derivative is needed for an interior solution in Section 3.1.
} 
Histories and feasible strategies The events in period $t$ are denoted by $h_{t}=$ $\left(d_{t}^{P}, w_{t}, d_{t}^{A}, e_{t}, b_{t}\right)$, with $h_{t}$ being public information. A history of length $t-1, h^{t-1}$ (for $t \geq 2$ ) collects the events up to, and including, time $t-1$, i.e. $h^{t-1}:=\left(h_{\tau}\right)_{\tau=1}^{t-1}$. The set of histories of length $t-1$ is denoted by $\mathscr{H}^{t-1}$ (and $\mathscr{H}^{0}=\{\emptyset\}$ ). I focus on pure strategies. For the agent, a pure strategy specifies what wage offers to accept in each period as a function of the previous history, and what level of effort to exert as a function of the previous history and current-period wages. Formally, it is a sequence of mappings $\left\{\sigma_{t}^{A}\right\}_{t=1}^{T}$ where, for each $t \leq T, \sigma_{t}^{A}=\left(d_{t}^{A}, e_{t}\right)$, and $d_{t}^{A}: \mathscr{H}^{t-1} \times\{0,1\} \times$ $\mathbb{R}_{+} \rightarrow\{0,1\},\left(h^{t-1}, d_{t}^{P}, w_{t}\right) \mapsto d_{t}^{A}\left(h^{t-1}, d_{t}^{P}, w_{t}\right)$ and $e_{t}: \mathscr{H}^{t-1} \times\{0,1\} \times \mathbb{R}_{+} \times\{0,1\} \rightarrow$ $\mathbb{R}_{+},\left(h^{t-1}, d_{t}^{P}, w_{t}, d_{t}\right) \mapsto e_{t}\left(h^{t-1}, d_{t}^{P}, w_{t}, d_{t}\right)$.

In each period, a pure strategy for the principal specifies her wage offer as a function of the previous history as well as the bonus payment as a function of the previous history, current-period wages and effort. Formally, it is a sequence of mappings $\left\{\sigma_{t}^{P}\right\}_{t=1}^{T}$, where, for each $t \leq T, \sigma_{t}^{P}=\left(d_{t}^{P}, w_{t}, b_{t}\right)$, and $d_{t}^{P}: \mathscr{H}^{t-1} \rightarrow\{0,1\},\left(h^{t-1}\right) \mapsto d_{t}^{P}\left(h^{t-1}\right)$, $w_{t}: \mathscr{H}^{t-1} \times\{0,1\} \rightarrow \mathbb{R}_{+},\left(h^{t-1}, d_{t}^{P}\right) \mapsto w_{t}\left(h^{t-1}\right), b_{t}: \mathscr{H}^{t-1} \times\{0,1\} \times \mathbb{R}_{+} \times\{0,1\} \times$ $\mathbb{R}_{+} \rightarrow \mathbb{R}_{+},\left(h^{t-1}, d_{t}^{P}, w_{t}, d_{t}, e_{t}\right) \mapsto b_{t}\left(h^{t-1}, d_{t}^{P}, w_{t}, d_{t}, e_{t}\right)$.

Relational Contract and the Norm of Reciprocity The relational contract is agreed upon at the beginning of the game. It "activates" the norm of reciprocity and stipulates reference functions which specify history-dependent actions players are supposed to take. For the agent, the relational contract determines an acceptance function $\hat{d}_{t}^{A}\left(h^{t-1}, d_{t}^{P}, w_{t}\right)$ as well as an effort function $\hat{e}_{t}\left(h^{t-1}, d_{t}^{P}, w_{t}, d_{t}\right)$, with $\left(\hat{d}_{t}, \hat{e}_{t}\right) \in \sigma_{t}^{A}$. For the principal, the relational contract determines an offer function $\hat{d}_{t}^{P}\left(h^{t-1}\right)$, a wage function $\hat{w}_{t}\left(h^{t-1}, d_{t}^{P}\right)$ and a bonus function $\hat{b}_{t}\left(h^{t-1}, w_{t}, d_{t}, e_{t}\right)$, with $\left(\hat{d}_{t}^{P}, \hat{w}_{t}, \hat{b}_{t}\right) \in \sigma_{t}^{P}$.

The norm of reciprocity states how the agent is supposed to reciprocate against "nondiscretionary" upfront wages, that is, wages that are not paid as a reward for past effort. To incorporate this notion, the total wage $\hat{w}_{t}\left(h^{t-1}, d_{t}^{P}\right)$ is split into a discretionary part $\hat{w}_{t}^{d}\left(h^{t-1}, d_{t}^{P}\right)$ and a "non-discretionary" component, which is defined as $\hat{w}_{t}^{n d}\left(h^{t-1} \backslash\left\{e^{t-1}, d^{A, t-1}\right\}, d_{t}^{P}\right)$, where $e^{t-1}:=\left(e_{\tau}\right)_{\tau=1}^{t-1}$ and $d^{A, t-1}:=\left(d_{\tau}^{A}\right)_{\tau=1}^{t-1}$. Note that this specification does not rule out an indirect relationship between the agent's effort and $w_{t}^{\text {nd }}$, since the latter is a function of the principal's previous actions which itself are affected by the agent's behavior. Whereas the bonus and discretionary wage constitute the "direct" incentive system that grants payments as a reward for previously exerted effort, $w_{t}^{\text {nd }}$ stipulates subsequent effort by the agent who adheres to the norm of reciprocity.

The agent's utility function also determines his responsiveness to the norm of reciprocity and - for period $t$ - equals 


$$
u_{t}=d_{t}\left(b_{t}+w_{t}-c\left(e_{t}\right)+\eta_{t} w_{t}^{n d} e_{t} \theta\right) .
$$

The term $\eta_{t} \in[0, \infty)$ captures the agent's inherent preferences for positive reciprocity (negative reciprocity is considered in Section A.2 in Appendix A) and lets the principal's output enter the agent's utility whenever $w_{t}^{\text {nd }}>0 .^{5}$ Its value in a given period depends on the history via a norm function, which takes the following form: When the relational contract is agreed upon at the beginning of the game, the reciprocity parameter is activated with $\eta_{1}=\eta \geq 0$. The value $\eta$ depends on the agent's individual characteristics, but also on the match-specific relationship between the principal and the agent. ${ }^{6} \eta_{t}$ remains at $\eta$ if the principal's actions so far have been consistent with the reference functions specified by the relational contract. Otherwise, it drops to and remains at zero in all subsequent periods. ${ }^{7}$ Therefore, in all periods $t \geq 2$,

$$
\eta_{t}= \begin{cases}\eta & \text { if } d_{\tau}^{P}=\hat{d}_{\tau}^{P}\left(h^{\tau-1}\right), b_{\tau} \geq \hat{b}\left(h^{\tau-1}, d_{\tau}^{P}, w_{\tau}, d_{\tau}^{A}, e_{\tau}\right), w_{\tau} \geq \hat{w}\left(h^{\tau-1}, d_{\tau}^{P}\right), \text { all } \tau \leq t \\ 0 & \text { otherwise }\end{cases}
$$

Note that $\eta_{t}$ does not drop to zero after a deviation by the agent (and if no bonus is paid in response), capturing the idea that the agent's general "goodwill" towards the principal depends on the latter's behavior, not on his own. Hence, the agent's reciprocal inclinations towards the principal disappear once the latter refuses to make an offer she was supposed to make, or if she does not compensate the agent accordingly. This also includes deviations with respect to $w_{t}^{\text {nd }}$ because otherwise, the principal could potentially reduce the non-discretionary wage after a deviation by the agent, which would contradict the definition of $w_{t}^{\text {nd }}$ to be independent of the agent's previous effort.

The principal has no preferences for reciprocity and only maximizes her material payoffs,

$$
\pi_{t}=d_{t}\left(e_{t} \theta-b_{t}-w_{t}\right)
$$

Now, a subgame perfect equilibrium determines equilibrium functions $d_{t}^{P}\left(h^{t-1}\right), w_{t}\left(h^{t-1}, d_{t}^{P}\right)$, $d_{t}^{A}\left(h^{t-1}, d_{t}^{P}, w_{t}\right), e_{t}\left(h^{t-1}, d_{t}^{P}, w_{t}, d_{t}^{A}\right)$, and $b_{t}\left(h^{t-1}, d_{t}^{P}, w_{t}, d_{t}^{A}, e_{t}\right)$. In addition, for every history, I impose the consistency requirements $\hat{d}_{t}^{P}=d_{t}^{P}, \hat{w}_{t}=w_{t}, \hat{d}_{t}^{A}=d_{t}^{A}, \hat{e}_{t}=e_{t}$, and

\footnotetext{
${ }^{5}$ In a more general setting, the norm of reciprocity would be activated if $w_{t}^{\text {nd }}$ exceeded some reference wage. Here, such a reference wage would equal zero; in Section 5.1, I consider positive reference wages.

${ }^{6}$ For example because the agent develops sentiment for the principal when working for her (Akerlof, 1982).

${ }^{7}$ Note that a drop to zero is not required. Any reduction of $\eta$ after a deviation by the principal would yield similar results. Also note that this definition can equivalently be applied to settings in which the principal does not observe effort and output is not verifiable. Then, the bonus could be a function of output, and $\eta_{t}$ would drop to zero if the principal reneged on paying it.
} 
$\hat{b}_{t}=b_{t}$. Incorporating these restrictions to a subgame perfect equilibrium, I denote the resulting equilibrium as a subgame norm-perfect equilibrium (SNPE).

In an SNPE in which $d_{t}^{P}=d_{t}^{A}=1$ in all the periods of the game, the following recursive relationships hold in all periods $t \in\{1, \ldots, T\}$ for the principal's profits $\Pi_{t}$ and the agent's utility $U_{t}$, where I set $\Pi_{T+1}=U_{T+1}=0$ :

$$
\begin{aligned}
\Pi_{t} & =e_{t} \theta-b_{t}-w_{t}+\delta \Pi_{t+1} \\
U_{t} & =b_{t}+w_{t}-c\left(e_{t}\right)+\eta_{t} w_{t}^{n d} e_{t} \boldsymbol{\theta}+\delta U_{t+1} .
\end{aligned}
$$

In what follows, the objective is to characterize an SNPE that maximizes the principal's profits at the beginning of the game, $\Pi_{1}$.

Before characterizing such a profit-maximizing SNPE in Section 3, I discuss the assumptions made with respect to the agent's preferences for reciprocity and the finite horizon of the game.

\subsection{Discussion of Assumptions}

Reciprocity I build on the gift exchange idea by Akerlof (1982) and assume that the principal can strategically "use" the norm of reciprocity. The agent understands the purpose of a gift received by the profit-maximizing principal but still reciprocates. This presumption is supported by experimental evidence presented by Malmendier and Schmidt (2017), who show that subjects reciprocate to gifts even though they apprehend that the giver is selfish and expects something in return.

The agent's responsiveness to the norm of reciprocity, the value $\eta_{t}$, depends on whether realized behavior deviates from the reference functions determined by the relational contract. Thus, the "behavioral" component of the agent's preferences is not only a function of the principal's past actions, but also of equilibrium behavior. In addition to the empirical literature showing that norms and social preferences may respond to the history of a relationship (as discussed in the Introduction), ${ }^{8}$ this approach is inspired by Cox et al. (2008). They assume that an action by one player is perceived as more (less) generous - and consequently causes a stronger reciprocal reaction - if it allows the other player to obtain a higher (lower) monetary payoff (Cox et al. (2008), Definitions 1 and 2; Axiom R). Furthermore, (positive or negative) reciprocal reactions are stronger whenever an action upsets the status quo compared with this same action if it only upholds the status quo (Cox et al. (2008), Axiom S, Part 1). I capture the first aspect by

${ }^{8}$ This view is also related to identity economics which argues that individuals' preferences should be modelled as a function of social context (Akerlof and Kranton, 2000; Akerlof and Kranton, 2005). 
assuming that realized payments trigger reciprocal behavior by the agent. Concerning the second aspect, I account for the "standard" role of a relational contract in the sense that it establishes a direct incentive system in which payments are promised in return for effort. Hence, wages and bonuses paid as a reward for past effort do not trigger reciprocal behavior, and $\eta$ drops to zero after the principal refuses to compensate the agent for his performance. I also show that the latter is not needed if negative reciprocity is explicitly considered (Section A.2 in Appendix A) and that my main results are robust to letting the agent's reciprocal preferences respond to all payments (Sections 5.2 and A.4 in Appendix A). Although my approach might at first sight appear more complicated than a setting in which the agent reciprocates to all payments (i.e., also performance pay the agent is bound to receive anyway), it actually simplifies the analysis of the dynamic relational contract because it allows for a recursive structure of the optimization problem (see Lemma 2). ${ }^{9}$

Moreover, $\eta$ drops to zero only after a deviation by the principal but not by the agent, hence the agent's general willingness to reciprocate gifts from the principal only depends on the latter's behavior. Otherwise, the agent would directly punish himself with a deviation, an implication I want to rule out.

Furthermore, I assume that reciprocity only enters the agent's stage game payoffs. This notion is consistent with evidence delivered by Bellemare and Shearer (2009), who show that a gift causes a positive effort response - but that this effect is only temporary. In Section A.3 in Appendix A, I also analyze a situation in which a positive wage today increases tomorrow's reference wage.

The reciprocity term in the agent's utility function contains $\theta$, representing the extent to which the principal benefits from the agent's effort. This is in line with evidence that an important factor for reciprocity is the agent's assessment of the value generated for the principal (Hennig-Schmidt et al., 2010; Englmaier and Leider, 2012b).

Finally, I assume that the principal knows $\eta$. In Section 5.3, I explore the consequences of asymmetric information concerning the agent's preferences for reciprocity.

Finite Time Horizon I analyze a game of $T$ periods, and most of the results on the dynamics of the employment relationship rely on the time horizon being finite (however note that the benefits of committing to future non-discretionary wages, as derived in Section 4, would also materialize in an infinite-horizon setting). Whereas many real-

\footnotetext{
${ }^{9}$ One could also argue that, if performance pay was very generous in relation to the agent's effort cost, it should be regarded as a gift. However, such a payment could be split into a part that compensates the agent for his effort costs and one that grants him a rent. Paying this rent up front (and anticipating that the agent exerts effort accordingly) would then be equivalent to paying a non-discretionary wage in my setting.
} 
life employment relationships either have a pre-defined ending date or an increasing probability of termination (which could be captured by a decreasing discount factor and generate the same dynamics, because those rely on the gradual reduction of future profits), most people work in multi-worker firms that continue to exist when workers retire. In my setting, this would imply that the principal also has the option to hire other agents for the job under consideration - after period $T$ or potentially even before. Taking this into account, my results survive as long as multilateral punishments are not feasible (e.g., because deviations in one relationship cannot be observed by other (prospective) employees): With multilateral punishments, the principal's commitment in the employment relationship would not necessarily be smaller in the later periods of an employment relationship (which drives the dynamics in my setting). However, although deviations have to be "private" information of one match to render multilateral relational contracts (as in Levin, 2002) unfeasible, it would be fine for outsiders to observe whether the agent is employed or is fired. Then, only a premature termination could be punished by any "new" agent. This would make it costly for the principal to replace the agent early on, leaving my results valid.

If I completely ruled out punishments by prospective new agents in the case of a premature termination, the opportunity to employ other agents would manifest in a positive outside option for the principal. Then my results remain as long as this outside option is sufficiently small for the principal to never have an incentive to terminate an employment relationship on the equilibrium path. This could be due to replacement costs when hiring a new agent, like search costs or other labor market frictions, or direct replacement costs.

\section{Results}

\subsection{Reciprocity Spot Contract}

I first derive a profit-maximizing spot contract and hence omit the time subscripts. Besides serving as a benchmark, such a contract will also be offered in the final period, $T$ (this follows from Lemma 2 below). In a spot contract, $b=0$ because the principal has no incentive to make a payment to the agent after the latter has exerted effort. Therefore, the only means to incentivize the agent is a positive non-discretionary wage. Since $w=w^{n d}$, I omit the " $n d$ " superscript in this section. Given $w$, and presuming he decides to work for the principal, the agent chooses effort to maximize his per-period utility $u=w-e^{3} / 3+\eta w e \theta$.

The conditions for using the first order approach hold, and thus the agent's incentive 
compatibility (IC) constraint yields

$$
e^{*}=\sqrt{\eta w \theta}
$$

The principal sets $w$ to maximize her expected per-period profits $\pi=e^{*} \theta-w$. Here, she has to take into account that accepting the contract must be optimal for the agent. This is captured by the agent's individual rationality (IR) constraint,

$$
w-\frac{\left(e^{*}\right)^{3}}{3}+\eta w e^{*} \theta \geq 0 .
$$

Concluding, the principal's problem is to

$$
\max _{w} e^{*} \theta-w
$$

subject to (IR) and (IC) and the non-negativity constraint $w \geq 0$.

Lemma 1 The profit-maximizing reciprocity spot contract has $w=\eta \theta^{3} / 4$ and $e^{*}=$ $\eta \theta^{2} / 2$. Therefore, $\pi=\eta \theta^{3} / 4$ and $u=\eta \theta^{3} / 4+\eta^{3} \theta^{6} / 12$.

The proof can be found in Appendix B.

Intuitively, a positive wage lets the agent partially internalize the principal's payoff, which is why he reciprocates and selects a positive effort level. Because this interaction is stronger for a more reciprocal agent, a higher $\eta$ induces larger values of $w, e^{*}, \pi$, and $u$.

\subsection{Relational Contract}

Now, I analyze how a relational contract is used to incentivize the agent. Two aspects are of particular interest, namely the enforceability of the relational contract and how the norm of reciprocity affects outcomes.

\subsubsection{Preliminaries and Optimization Problem}

The relational contract determines payment functions $w_{t}^{d}\left(h^{t-1}, d_{t}^{P}\right), w_{t}^{n d}\left(h^{t-1} \backslash\left\{e^{t-1}, d^{A, t-1}\right\}, d_{t}^{P}\right)$, and $b_{t}\left(h^{t-1}, d_{t}^{P}, w_{t}, d_{t}^{A}, e_{t}\right)$. The promise to make these payments must be credible, which is captured by dynamic enforcement (DE) constraints for each period $t$,

$$
-b_{t}+\delta \Pi_{t+1} \geq \delta \tilde{\Pi}_{t+1}
$$


as well as individual rationality (IR) constraints, $\Pi_{t} \geq \tilde{\Pi}_{t}$. Because $b_{t} \geq 0$, (IR) are implied by (DE) constraints and can hence be omitted. $\Pi_{t+1}$ describes the principal's on-path and $\tilde{\Pi}_{t+1}$ her off-path continuation profits. The (DE) constraint indicates that discretionary payments are only feasible if $\Pi_{t+1}>\tilde{\Pi}_{t+1}$, i.e., if future equilibrium play can be made contingent on the principal's current behavior. Generally, relational contracts require a (potentially) infinite time horizon because of a standard unraveling argument that can be applied once a predetermined last period exists: If the equilibrium outcome in the last period is unique, the same holds for all preceding periods. In my case, however, the situation is different because the norm function lets $\eta_{t}$ drop to zero once the principal refuses to make a specified payment. Moreover, the "standard" grim trigger punishment is imposed afterward and relational contracts are no longer feasible (adapting Abreu (1988) to my setting in the sense that any obseravable deviation from agreed upon behavior should be punished by a reversion to a player's minmax payoff). This implies that the principal's continuation profits are $\tilde{\Pi}_{t+1}=0$ if she has deviated in any $\tau \leq t$, and her behavior in period $t<T$ indeed affects her future profits. Hence, not only does the relational contract determine whether a given payment "activates" the agent's reciprocal preferences, but the latter are also a prerequisite for the relational contract to work.

In the next step, I explore the agent's incentives to exert equilibrium effort. Those are generally determined by a combination of reciprocity-based incentives (via a positive $w_{t}^{n d}$ ) and relational incentives (via $b_{t}$ and $w_{t}^{d}$ ). Recall that my specification of the norm function implies that after a deviation by the agent, the reciprocity parameter remains at $\eta$. This indicates that the agent does not necessarily deviate to an effort level of zero. Moreover, since effort is public information, it is without loss to only specify a positive bonus $b_{t} \geq 0$ if the agent has exerted equilibrium effort and no bonus otherwise. Thus, the agent's (IC) constraint (which must hold in every period $t$ ) equals

$$
-\frac{\left(e_{t}^{*}\right)^{3}}{3}+\eta w_{t}^{n d} e_{t}^{*} \theta+b_{t}+\delta U_{t+1} \geq-\frac{\left(\tilde{e}_{t}\right)^{3}}{3}+\eta w_{t}^{n d} \tilde{e}_{t} \theta+\delta \tilde{U}_{t+1},
$$

where $\tilde{U}_{t+1}$ is the agent's continuation utility after a deviation by himself. Moreover, if the agent deviates, he will choose an effort level $\tilde{e}_{t}=\operatorname{argmax}\left(-e^{3} / 3+\eta w_{t}^{\text {nd }} e \theta\right)$, i.e., $\tilde{e}_{t}=\sqrt{\eta w_{t}^{n d} \theta} . \tilde{e}_{t}$ is the effort the agent would select if he only responded to the norm of reciprocity. Relational incentives using subsequent discretionary payments are needed to motivate the agent to exert additional effort $e_{t}^{*}-\tilde{e}_{t}$.

An (IR) constraint $U_{t} \geq 0$ must also hold in every period but is implied by (IC) because payments are assumed to be non-negative and because the right-hand side of (IC) cannot be smaller than zero. 
Concluding, the principal's problem is to maximize

$$
\Pi_{1}=\sum_{t=1}^{T} \delta^{t-1} \pi_{t}
$$

subject to a (DE) and (IC) constraints for every period $t$.

First, I derive a number of preliminary results, which substantially simplify the problem and are collected in Lemma 2.

Lemma 2 There exists a profit-maximizing equilibrium which has the following properties in all periods $t$ :

- $w_{t}=w_{t}^{n d}$

- (IC) holds as an equality

- $U_{t}=\tilde{U}_{t}$

- the equilibrium is sequentially optimal, hence the problem is equivalent to maximizing each $\pi_{t}$

The proof can be found in Appendix B.

First, it is without loss of generality to only use bonus payments for the provision of relational incentives. Thus, $w_{t}=w_{t}^{n d}$ for the remainder of the paper, and all upfront wages are non-discretionary. This simplifies the separation of "standard" direct incentives (which are denoted "relational incentives" and provided by $b_{t}$ ) from those relying on the norm of reciprocity (denoted "reciprocity-based incentives" and provided by $w_{t}$ ).

Second, the (IC) constraint binds in every period. If it did not bind, the bonus $b_{t}$ could be slightly reduced, which would increase profits and relax the (DE) constraint without violating the (IC) constraint. Thus, the agent does not receive a rent for relational incentives. However, he enjoys a rent whenever $w_{t}\left(=w_{t}^{n d}\right)>0$, i.e., when reciprocitybased incentives are provided. Importantly, though, these "warm-glow" rents cannot be used to provide relational incentives in earlier periods: If the agent was bound to lose them after a deviation (for example because of a firing threat as with efficiency wages), the upfront wage would not be non-discretionary anymore, and the agent would not reciprocate. ${ }^{10}$

\footnotetext{
${ }^{10}$ This would be different if either bonus or discretionary wages also triggered direct reciprocal responses by the agent. Then, the respective payments would merely assume a larger relative weight in the optimal incentive scheme (see Sections 5.2 and A.4 in Appendix A).
} 
Third, $U_{t}=\tilde{U}_{t}$ then follows because it is without loss of generality to provide relational incentives only with a current bonus. Thus, continuation play is not affected by the agent's actions, which finally implies that the profit-maximizing relational contract is sequentially optimal and the optimization problem has a recursive structure.

Collecting all results, binding (IC) constraints as well as $U_{t}=\tilde{U}_{t}$ yield $b_{t}=e_{t}^{3} / 3-$ $\eta w_{t} e_{t} \theta+2 / 3\left(\sqrt{\eta w_{t} \theta}\right)^{3}$. Plugging this into the principal's profits and (DE) constraints, the optimization problem becomes to maximize

$$
\pi_{t}=e_{t} \theta-b_{t}-w_{t}=e_{t} \theta-\left(\frac{e_{t}^{3}}{3}-\eta w_{t} e_{t} \theta+\frac{2}{3}\left(\sqrt{\eta w_{t} \theta}\right)^{3}\right)-w_{t}
$$

in every period $t$, subject to

$$
\frac{e_{t}^{3}}{3}-\eta w_{t} \theta e_{t} \leq \delta \Pi_{t+1}-\frac{2}{3}\left(\sqrt{\eta w_{t} \theta}\right)^{3}
$$

\subsubsection{Reciprocity-Based Incentives in a Relational Contract}

In this section, I derive the conditions for the use of reciprocity-based incentives in a relational contract. These are based on the following trade-off which the principal faces. Relational incentives allow her to extract the full surplus, reciprocity-based incentives grant the agent a rent but reduce his effective effort costs. The optimal relational contract balances the costs and benefits of both means to provide incentives, taking into account that the (DE) constraint restricts the use of relational incentives.

First, I abstract from issues of enforceability and assume that the (DE) constraint does not bind (i.e., is satisfied for the principal's preferred effort level). This situation is equivalent to one in which formal contracts based on effort would be feasible.

Lemma 3 Assume the $(D E)$ constraint does not bind in period $t<T$. Then, there exists $a \bar{\eta}>0$ such that setting a strictly positive wage is optimal for $\eta>\bar{\eta}$, whereas the optimal wage is zero for $\eta \leq \bar{\eta}$.

The proof can be found in Appendix B.

Lemma 3 implies that even if the principal's discounted future on-path profits are sufficiently large to not restrict her in setting the most preferred effort-based bonus $b_{t}$, she might still decide to grant the agent a rent if $\eta$ is sufficiently large. In the following, I refer to the effort and wage levels for a non-binding (DE) constraint as the first-best 
levels:

$$
\begin{aligned}
e^{F B}=\frac{1+\eta^{2} \theta^{3}}{2 \eta \theta}, w^{F B} & =\frac{\left(\eta^{2} \theta^{3}-1\right)^{2}}{4 \eta^{3} \theta^{3}} \text { if } \eta>\bar{\eta} \\
e^{F B} & =\sqrt{\theta}, w^{F B}=0 \text { if } \eta \leq \bar{\eta} .
\end{aligned}
$$

In the next step, I assess how the agent's preferences for reciprocity affect the outcomes if her (DE) constraint binds.

Lemma 4 Assume the $(D E)$ constraint binds in period $t<T$. Then, equilibrium effort is smaller than with a non-binding (DE) constraint. Moreover, if paying a fixed wage is optimal in the situation with a non-binding $(D E)$ constraint (i.e., if $\eta>\bar{\eta}$ ), the fixed wage now is strictly larger. Otherwise (i.e., if $\eta \leq \bar{\eta}$ ), there exists a $\tilde{\eta}_{t}<\bar{\eta}$ such that setting a strictly positive wage is optimal for $\eta>\tilde{\eta}_{t}$, whereas the optimal wage equals zero for $\eta \leq \tilde{\eta}_{t}$. Finally, $\tilde{\eta}_{t}$ is increasing in $\delta$.

The proof can be found in Appendix B.

Besides reducing effective effort costs, an upfront wage also relaxes the principal's (DE) constraint by decreasing the bonus that must be paid for implementing a given effort level. Therefore, if the (DE) constraint binds, the wage is generally larger than when it does not bind.

This implies that reciprocity-based incentives can improve the power of relational incentives for a given value of $\eta$, and vice versa (this complementarity between the two means to provide incentives is further fueled by a positive effect of $\eta$ on future profits, see Proposition 2).

\subsubsection{Relational and Reciprocity-Based Incentives as Dynamic Substitutes}

In this section, I characterize how, for a given value of $\eta$, the interaction between relational and reciprocity-based incentives evolves. The (DE) constraint might or might not bind in any period $t<T$ depending on discount factor $\delta$, reciprocity parameter $\eta$, and productivity $\theta$. Furthermore, the (DE) constraint becomes tighter in later periods.

Lemma 5 For every $\delta>0$, the (DE) constraint in period $T$ - 1 holds for first-best effort and wage levels if $\eta$ is sufficiently large. For any values $\eta$ and $\theta$, the (DE) constraint in period T-1 does not hold for first-best effort and wage levels if the discount factor is sufficiently small.

Furthermore, $\Pi_{t-1}>\Pi_{t}$ for all $t \leq T$. 
The proof can be found in Appendix B.

The principal's commitment in a relational contract is determined by what she has to lose given she deviates. If the discount factor is small, she cares less about a potential reduction in future profits and is therefore less willing to pay a bonus to compensate the agent for his effort. Conversely, a larger reciprocity parameter $\eta$ increases future profits and reduces today's effective effort costs. The second part of Lemma 5 states that on-path profits decline over time. As time passes, the remaining time horizon and consequently the number of periods in which profits can be generated falls. This also triggers a reinforcing effect. Since $\Pi_{T}>0$, the (DE) constraint allows a larger effort level in period $T-1$ than in period $T$. Then, per-period profits in period $T-1$ are higher than those in period $T$, and implementable effort in period $T-2$ is even larger than in period $T-1$, and so on.

All this implies that if the (DE) constraint binds in a given period $\tilde{t}$, it will also bind in all subsequent periods $t>\tilde{t}$. If it is slack in a given period $\hat{t}$, it will also be slack in all previous periods $t<\hat{t}$. This yields the following effort and compensation dynamics.

Proposition 1 Equilibrium effort is weakly decreasing over time, i.e., $e_{t}^{*} \leq e_{t-1}^{*}$. Moreover, $e_{t}^{*}<e_{t-1}^{*}$ implies $e_{t+1}^{*}<e_{t}^{*}$, whereas $e_{t+1}^{*}=e_{t}^{*}$ implies $e_{t}^{*}=e_{t-1}^{*}$.

The equilibrium wage is weakly increasing over time and the bonus weakly decreasing, i.e., $w_{t} \geq w_{t-1}$ and $b_{t} \leq b_{t-1}$. Moreover, $w_{t}>w_{t-1}$ and $b_{t}<b_{t-1}$ imply $w_{t+1}>w_{t}$ and $b_{t+1}<b_{t}$, whereas $w_{t+1}=w_{t}$ and $b_{t+1}=b_{t}$ imply $w_{t+1}=w_{t}$ and $b_{t}=b_{t-1}$.

The agent's total compensation, $w_{t}+b_{t}$, might increase or decrease over time.

The proof can be found in Appendix B.

Proposition 1 indicates that effort and compensation are time-invariant in the early stages of the employment relationship, as long as the future is sufficiently valuable for the (DE) constraint not to bind. Once the end of the employment relationship is close and the (DE) constraint binds, effort and bonus profiles become downward-sloping and the wage profile upward-sloping. This is because the principal can no longer credibly promise her preferred bonus. On the one hand, this reduces equilibrium effort. On the other hand, the principal might respond with a wage increase that raises equilibrium effort due to the agent's preferences for reciprocity. The effort increase caused by a higher wage does not fully compensate for the effort reduction caused by the binding (DE) constraint, though, because the costs of implementing one additional unit of effort are now higher with reciprocity-based incentives than with relational incentives. Over time, the (DE) constraint is further tightened (Lemma 5). 
Hence, toward the end of an employment relationship, relational incentives are gradually replaced by reciprocity-based incentives (bonus $\downarrow$, wage $\uparrow$ ), with the substitution however being incomplete (effort $\downarrow$ ). The dynamics of the agent's total compensation, $w_{t}+b_{t}$, are not necessarily monotone and depend on the relative importance of relational and reciprocity-based incentives.

Payoffs Whereas the principal's per-period profits decrease over time (once (DE) binds), the opposite is true for the agent's per-period utilities. This result is also driven by the gradual replacement of relational with reciprocity-based incentives; because of the binding (IC) constraint, the agent only collects a rent with the latter.

Lemma 6 The principal's per-period profits $\pi_{t}$ are weakly decreasing over time, i.e., $\pi_{t} \leq \pi_{t-1}$. Moreover, $\pi_{t}<\pi_{t-1}$ implies $\pi_{t+1}<\pi_{t}$, whereas $\pi_{t+1}=\pi_{t}$ implies $\pi_{t}=\pi_{t-1}$.

The agent's per-period utility $u_{t}$ is weakly increasing over time, i.e., $u_{t} \geq u_{t-1}$. Moreover, $u_{t}>u_{t-1}$ implies $u_{t+1}>u_{t}$, whereas $u_{t+1}=u_{t}$ implies $u_{t}=u_{t-1}$.

The proof can be found in Appendix B.

\subsubsection{Reciprocity}

In the previous sections, I have derived the properties of a profit-maximizing relational contract for a given value of $\eta$. Now, I explore how the agent's responsiveness to the norm of reciprocity affects effort during his career.

Proposition 2 Equilibrium profits $\Pi_{t}$ and effort $e_{t}$ increase in $\eta$. This positive effect is stronger if the (DE) constraint binds (i.e., in later periods).

The proof can be found in Appendix B.

First, a higher $\eta$ directly raises $e_{t}$ (and consequently profits) for a given $w_{t}>0$ due to the reduction in effective effort costs. Second, there is an indirect effect. Because future profits also increase in $\eta$, the (DE) constraint in period $t$ is relaxed, which further leads to higher effort and profits. This interaction provides an additional source for the complementarity between relational and reciprocity-based incentives. Moreover, Proposition 2 indicates that the positive effect of $\eta$ on effort is stronger if the principal's (DE) constraint binds (i.e., in the later stages of the agent's career). Then, the incentive system places more weight on reciprocal incentives and the role of $\eta$ intensifies (see Fahn et al., 2017, for evidence). 


\section{Long-Term Commitment}

After having derived the properties of a relational contract without any commitment, I now show that the ability to formally commit to future non-discretionary wage payments - a feature shared by many real-world employment relationships - can raise profits by increasing the principal's credibility when providing relational incentives.

I assume that, at the beginning of every period $t$, the principal not only can pay $w_{t}$, but in addition commit to wages $\hat{w}_{\tau \mid t}, \tau>t$, to be paid at the beginning of period $\tau$. These wages can be topped up in later periods, thus $\hat{w}_{\tau \mid t^{\prime}} \geq \hat{w}_{\tau \mid t} \forall \tau$ for $t^{\prime}>t$. When period $\tau$ comes, the principal can further increase the wage by $w_{\tau}-\hat{w}_{\tau}$, where $\hat{w}_{\tau}$ describes the highest committed-to wage for period $\tau$. Hence $w_{\tau}$ amounts to the total period- $\tau$ wage to which the agent fully reciprocates.

The principal's commitment to future wages affects the (DE) constraint, which now amounts to

$$
-b_{t}+\delta \Pi_{t+1} \geq-\sum_{\tau=t+1}^{T} \delta^{\tau-t} \hat{w}_{\tau \mid t},
$$

whereas the (IC) constraint is not affected.

Committing to future wages thus relaxes the (DE) constraint and consequently increases the principal's credibility in the relational contract. The reason is that the agent receives $\hat{w}_{\tau \mid t}$ in any case, irrespective of whether the principal has paid the period- $t$ bonus or not. However, the agent only reacts reciprocally to $\hat{w}_{\tau \mid t}$ in case $b_{t}$ has indeed been paid. Thus, the costs of a deviation go up for the principal, and her increased vulnerability allows her to credibly promise a higher bonus. ${ }^{11}$ The specifics of the norm function are crucial for this mechanism to work: if the agent's reciprocal inclinations did not drop after a deviation by the principal, commitment to future wages would affect on- and off-path profits to the same extent and thus not help with the provision of incentives.

Also note that commitment does not come with costs in my setting. Thus, there is no harm for the principal to commit to a wage she intends to pay anyway, thereby relaxing the (DE) constraint. I consider costs of commitment in Section A.1 in Appendix A and derive conditions for commitment to be optimal in such a case. Here, to precisely describe the benefits of commitment, I assume that if the principal is indifferent between committing to various future wages, she chooses the lowest one. For example, if in a period $t$ the (DE) constraint is satisfied for first-best effort and wage levels without any

\footnotetext{
${ }^{11}$ This mechanism is reminiscent of outcomes in Ramey and Watson (1997) or Englmaier and Fahn (2019). There, physical investments increase the future rent generated by the relational contract by more than reservation values. Thus, investments increase players' commitment, and 'overinvestments" can be optimal.
} 
commitment, I assume that the principal chooses $\hat{w}_{\tau \mid t}=0 \forall \tau>t$. In Proposition 3, I thus characterize situations when commitment is strictly optimal, together with the according wages $\hat{w}_{\tau \mid t}$.

Proposition 3 Assume the principal can commit to future wages and that, if the principal is indifferent between different levels of $\hat{w}_{\tau \mid t}$, she chooses the lowest one.

Commitment to future wages might or might not be optimal in any period. If it is not optimal in a period $\bar{t}$, it also is not optimal in all previous periods $t<\bar{t}$. If commitment to future wages is optimal in a period $\tilde{t}$, it also is optimal in all subsequent periods $t>\tilde{t}$.

If $(D E)$ binds in a period $t$, it also binds in all subsequent periods. Then, $\hat{w}_{\tau \mid t}=w_{\tau}$ in all periods $\tau>t$, with $w_{\tau^{\prime \prime}} \geq w_{\tau^{\prime}}$ for $\tau^{\prime \prime}>\tau^{\prime}$ and a strict inequality if $w_{\tau^{\prime}}>0$.

The proof can be found in Appendix B.

Proposition 3 states that the optimal extent of commitment goes up over time. For example, in the early periods of an employment relationship, the (DE) constraint might hold for first-best wages/effort even without commitment. As time proceeds, the principal's future profits go down, and committing to future wages can counteract the tightening of effect of lower future profits. Eventually (DE) will bind, and it might become optimal to commit to wages which exceed the sequentially optimal levels, i.e., the wages that would be optimal from the perspective of a given period. Then, the wage structure is also upward sloping because the (DE) constraint is automatically tightened as time moves on.

Depending on parameter values and the time horizon, commitment might be optimal from the beginning, but it may also never needed, namely if profits and discount factor are sufficiently high to implement first-best levels anyway.

Finally, note that I allow the principal to commit to all future wages, thus once a wage $\hat{w}_{\tau}$ has been set she is not able to get out of such an obligation. Naturally, although some commitment arguably is possible in most occupations, it does not necessarily extend to an employee's entire career. This aspect is not instrumental for my results, though: if the principal has some commitment power, she will use it whenever she is constrained in the use of relational incentives, hence particularly at the end of an employee's career.

\section{Extensions and Robustness}

In the following, I analyze a number of extensions and the resulting implications for an optimal relational contract without commitment. Further extensions are explored in Appendix A. 


\subsection{General Reference Wage}

In the main part of this paper, the agent reciprocates to a positive non-discretionary wage. In this section, I assume that the wage must exceed some reference wage $\bar{w} \geq 0$ - for the agent to reciprocate and to accept the principal's employment offer. Besides serving as a robustness check, this section can also yield insights into the role of labor market competition or aspects such as the unemployment rate or unemployment benefits. For example, I would expect $\bar{w}$ to be higher with more competition for workers (as in Schmidt, 2011), or to be lower with a higher unemployment rate.

For simplicity, I assume $\bar{w}$ to be constant and not vary over time. Then, the agent's period- $t$ utility amounts to

$$
u_{t}=w_{t}+b_{t}+\eta_{t}\left(w_{t}-\bar{w}\right) \theta e_{t}-\frac{e_{t}^{3}}{3}
$$

First, I characterize effort and wage in a spot reciprocity contract.

Lemma 7 Effort in the profit-maximizing spot reciprocity contract is independent of $\bar{w}$. Moreover, $\partial w / \partial \bar{w}=1$ and $\partial e / \partial \bar{w}=0$.

The proof can be found in Appendix B.

The principal responds to a higher $\bar{w}$ with an increase in $w_{t}$ to keep incentives constant. Therefore, factors such as labor market competition or the unemployment rate do not affect the importance of the norm of reciprocity for the optimal provision of incentives in a static setting. $\bar{w}$ only causes a redistribution of rents.

To derive a profit-maximizing relational contract with positive outside options, I first characterize the agent's (IC) constraint for a general $\bar{w} \geq 0$ :

$$
b_{t}-\frac{\left(e_{t}\right)^{3}}{3}+\eta\left(w_{t}-\bar{w}\right) e_{t} \theta \geq \frac{2}{3}\left(\sqrt{\eta\left(w_{t}-\bar{w}\right) \theta}\right)^{3} .
$$

The outside wage $\bar{w}$ enters the agent's (IC) constraint only via the associated increase in the reference wage. This is different from a "standard" efficiency wage effect, where a better outside option of an employee directly reduces his incentives to work hard.

The principal's (DE) constraint still amounts to

$$
-b_{t}+\delta \Pi_{t+1} \geq 0 .
$$

The general structure of a profit-maximizing relational contract is as in my main model, with constant wage and effort levels as long as (DE) is slack as well as upward- 
sloping wage and downward-sloping effort profiles once (DE) becomes binding. Still, $\bar{w}$ crucially affects the importance of reciprocity-based incentives, as described in Proposition 4.

Proposition 4 A larger $\bar{w}$ tightens the $(D E)$ constraint. If $(D E)$ does not bind in period $t<T, \partial w_{t} / \partial \bar{w}=1$ and $\partial e_{t} / \partial \bar{w}=0$. If $(D E)$ binds in period $t<T, \partial w_{t} / \partial \bar{w}>1$ and $\partial e_{t} / \partial \bar{w}<0$.

Finally, the effort and compensation dynamics are as in Proposition 1

The proof can be found in Appendix B.

As a larger value of $\bar{w}$ has no direct effect on the optimal provision of incentives, the principal implements the same effort for all values of $\bar{w}$ if (DE) does not bind (i.e., in earlier periods of the employment relationship). Then, as in a reciprocity spot contract, a higher $\bar{w}$ causes a mere redistribution of rents from the principal to the agent (and $\left.\partial w_{t} / \partial \bar{w}=1\right)$.

However, a higher value of $\bar{w}$ reduces the principal's future profits. This tightens the (DE) constraint and, once the constraint binds, restricts the principal's possibility of using relational incentives. As in the main analysis (see Lemma 1 and Proposition 1), she mitigates the necessary effort reduction by expanding reciprocity-based incentives and raising $w_{t}$ beyond the increase induced by a larger $\bar{w}$. Hence, $\partial w_{t} / \partial \bar{w}>1$ if (DE) binds.

Now, if the labor market becomes more competitive and $\bar{w}$ consequently goes up, the (DE) constraint binds earlier, effort goes down and wages go up. ${ }^{12}$ This is driven by the reduced relationship surplus and thus commitment in the relational contract, letting reciprocity-based incentives become more important in a profit-maximizing dynamic incentive scheme. This result relates to a number of theoretical and empirical contributions which have analyzed the effect of competition on the role of social preferences. If contracts are complete, competition has been found to drive out social preferences (Fehr and Schmidt, 1999; Dufwenberg et al., 2011). With incomplete contracts (such as in the present setting), the situation is different, though (Fehr and Fischbacher, 2002; Schmidt, 2011). Schmidt (2011) analyzes how labor market competition might affect the utilization of fairness preferences by firms. Applying a static model, he shows that induced effort levels are the same for all degrees of competition and that only rents are shifted between firms and workers (as in my setting with a spot contract). I demonstrate that, if the dynamic nature of employment relationships is taken into account, the

\footnotetext{
${ }^{12}$ The extent of labor market competition will also affect the principal's outside option; my results continue to hold as long as the effect on $\bar{w}$ is stronger.
} 
principal might actually make more use of reciprocity-based incentives in a more competitive labor market. Furthermore, the benefits of committing to future wages become more pronounced because the (DE) constraint generally is "tighter".

Moreover, there is evidence that a higher unemployment rate increases worker effort, which is in line with my results if one presumes a negative relationship between $\bar{w}$ and unemployment. Lazear et al. (2016) find that workers in a US firm exerted more effort and thus became more productive during periods of recession; moreover, effort was highest at establishments located in high-unemployment areas. Lazear et al. (2016) attribute this result to a firing threat for non-performance (which is also the basis for standard efficiency wage models such as Shapiro and Stiglitz, 1984). However, there is also evidence that firms are reluctant to use firing threats to discipline workers (Bewley, 1999). My approach thus can generate a positive interaction between effort and unemployment rate without a firing threat, based on a dynamic model of gift-exchange in the spirit of Akerlof (1982) (recall that $d e^{*} / d \bar{w}=0$ in the one-shot game). To empirically distinguish my mechanism from the ones derived in Lazear et al. (2016) or Shapiro and Stiglitz (1984), one could use that my model predicts a relationship between $\bar{w}$ and effort only in intermediate stages of an employment relationship. At the beginning of a career (if (DE) does not bind) or its very end (the last period), $d e^{*} / d \bar{w}=0$, a result not generated by the ones mentioned above.

\subsection{Reciprocity Triggered by all Current Payments}

Now, I let the agent's preferences for reciprocity be triggered by all realized current payments, for simplicity in a setting with $T=2$. Then, wages paid as a reward for previously exerted effort (and not only $w_{t}^{\text {nd }}$ ) also induce the agent to reciprocate. This does not hold for the bonus, however, which is paid after effort has been exerted (in Section A.4 in Appendix A, I let the norm of reciprocity extend to expected bonus payments). Therefore, only upfront wages are used to provide incentives because they can assume the role of the bonus and additionally induce reciprocal behavior.

To formally underpin this claim, the agent's second-period effort still maximizes $u_{2}=$ $w_{2}-c\left(e_{2}\right)+\eta w_{2} \theta e_{2} ;$ hence,

$$
e_{2}^{*}=\sqrt{\eta w_{2} \theta}
$$

Now, the principal does not maximize $\pi_{2}$ when selecting $w_{2}$, hence the profit-maximizing equilibrium is not sequentially optimal. Instead, $w_{2}$ is also a function of $e_{1}$ and set to maximize the principal's total discounted profit stream, $\Pi_{1}$. The agent's first-period effort must satisfy his (IC) constraint. Here, I assume that once the agent deviates, $b_{1}=0$, and $w_{2}$ is set such that $\pi_{2}$ is myopically maximized (in which case $w_{2}^{*}=\eta \theta^{3} / 4$, 
$e_{2}^{*}=\eta \theta^{2} / 2$, and $\left.u_{2}=\eta \theta^{3} / 4+\eta^{3} \theta^{6} / 12\right) .{ }^{13}$ Therefore, if the agent deviates, he chooses $\tilde{e}_{1}$ to maximize $\tilde{u}_{1}=w_{1}-e_{1}^{3} / 3+\eta w_{1} \theta e_{1}$, and thus $\tilde{e}_{1}=\sqrt{\eta w_{1} \theta}$.

All this implies that the agent's (IC) constraint equals

$$
\begin{aligned}
& b_{1}-\frac{e_{1}^{3}}{3}+\eta w_{1} \theta e_{1}^{*}+\delta\left[w_{2}+\frac{2\left(\sqrt{\eta w_{2} \theta}\right)^{3}}{3}\right] \\
\geq & \frac{2\left(\sqrt{\eta w_{1} \theta}\right)^{3}}{3}+\delta\left(\frac{\eta \theta^{3}}{4}+\frac{\eta^{3} \theta^{6}}{12}\right) .
\end{aligned}
$$

The principal is only willing to make equilibrium payments if her (DE) constraint holds,

$$
-b_{1}+\delta\left(e_{2} \theta-w_{2}\right) \geq 0 .
$$

Furthermore, $\eta$ drops to zero if $w_{2}$ differs from the amount promised at the beginning of period 1.

Then, the principal sets $w_{1}, w_{2}$, and $b_{1}$ to maximize $\Pi_{1}=e_{1}^{*} \theta-w_{1}-b_{1}+\delta\left(e_{2}^{*} \theta-w_{2}\right)$, subject to (IC) and (DE), and taking into account that $e_{2}^{*}=\sqrt{\eta w_{2} \theta}$.

The structure of the optimal arrangement is similar to that in the main part, with two exceptions. First, it is optimal to set $b_{1}=0$. On the contrary, assume a profitmaximizing equilibrium has $b_{1}>0$. Then, a reduction in $b_{1}$ by a small $\varepsilon>0$ together with an increase in $w_{2}$ by $\varepsilon / \delta$ does not affect (DE) and $\Pi_{1}$, but does relax (IC). Therefore, $w_{2}$ is above the level maximizing $\pi_{2}$ and bounded by the condition that secondperiod profits must be non-negative. This implies that the back-loading of upfront wages is more pronounced than before. Second, the principal's profits will be larger than those in the main model because the payments used to provide relational incentives also trigger reciprocal behavior, an aspect missing before.

However, it is not possible anymore to easily separate the two means to provide incentives because $w_{2}$ is not only used to provide reciprocity-based incentives in the second, but also for relational incentives in the first period. Put differently, the secondperiod "warm-glow" is also at the discretion of the agent's first-period effort and thus used to provide relational incentives.

Finally, note that allowing the principal to commit to non-discretionary future wages as in Section 4 also relaxes the (DE) constraint, which then becomes

$$
-b_{1}+\delta\left(e_{2} \theta-w_{2}+\hat{w}_{2}\right) \geq 0 \text {. }
$$

\footnotetext{
$\overline{{ }^{13} \text { Note that a firing threat would not be credible. }}$
} 


\subsection{Asymmetric Information}

So far, I have assumed that the principal is aware of the agent's $\eta$, for example because of personality tests used in the hiring process. In this section, I explore the potential implications of asymmetric information on the agent's reciprocal inclinations, again in a setting with $T=2$. I assume that the agent can either be a "reciprocal" type with $\eta>0$ (with probability $p \in(0,1)$ ) or a "selfish" type with no reciprocal preferences (with probability $1-p$ ). Moreover, the agent's type is his private information. Assuming that the principal can design the incentive scheme and does so in a profit-maximizing way, she chooses one of the following two options. First, the principal asks for a firstperiod effort level that only the reciprocal, but not the selfish agent is willing to exert. Then, the selfish agent collects the first-period wage, but is subsequently detected and fired (because he would exert no effort in the second period). I call this a "separation contract". Second, the effort request is sufficiently low that it satisfies the selfish type's (IC) constraint. In this case, the agent's effort choice cannot be used to screen agents and both types are also employed in the second period. Only then does the selfish agent - after collecting $w_{2}$ - shirk by exerting zero effort. I call this arrangement a "pooling contract".

I retain the setting of Section 5.2 where the norm of reciprocity is triggered by all realized payments. This simplifies the analysis of asymmetric information because, in a separation contract, the reciprocal agent takes into account that he will only remain employed if he exerts equilibrium effort in the first period. Therefore, his incentives to exert first-period effort are not only determined by the corresponding direct incentives, but also by the benefits of not being regarded as the selfish type. Taking this into account, only future wages are used to motivate the agent (Section 5.2).

Now, I derive a Perfect Bayesian Equilibrium where any deviation by the agent lets the principal assign probability 1 to facing the selfish type. Then, a separation and a pooling contract are both feasible. The (IC) constraints, one for the selfish type (ICS), and one for the reciprocal type (ICR), already taking into account that $e_{2}=\sqrt{\eta w_{2} \theta}$, amount to

$$
\begin{aligned}
& -\frac{e_{1}^{3}}{3}+\delta w_{2} \geq 0 \\
& -\frac{e_{1}^{3}}{3}+\eta w_{1} \theta e_{1}+\delta\left[w_{2}+\frac{2\left(\sqrt{\eta w_{2} \theta}\right)^{3}}{3}\right] \\
& \geq-\frac{\tilde{e}_{1}^{3}}{3}+\eta w_{1} \theta \tilde{e}_{1}
\end{aligned}
$$


with $\tilde{e}_{1}=\sqrt{\eta w_{1} \theta}$. Different from Section 5.2, a deviation from the equilibrium effort now results in a termination and henceforth zero off-path continuation utilities. For any effort level $e_{1} \geq \tilde{e}_{1}$ (ICS) is tighter than (ICR) (this is shown in the proof to Proposition 5). Therefore, if the principal offered the profit-maximizing contract designed for a reciprocal type (which involves a binding (ICR) constraint), this would automatically result in a separation of types. Moreover, effort in a pooling contract will be determined by a binding (ICS) constraint.

Proposition 5 In a profit-maximizing perfect Bayesian equilibrium at which any deviation from equilibrium effort induces the principal to assign probability 1 to facing a selfish type, a pooling contract is optimal if $p$ is sufficiently small. If $p$ is sufficiently large, a separating contract is optimal.

The proof can be found in Appendix B.

Generally, the principal faces the following trade-off. First, with a pooling contract, the first-period effort is rather low (determined by a binding (ICS) constraint); however, it is exerted by both types. Then, only the reciprocal type exerts effort in the second period, whereas both are paid $w_{2}$. In this case, the principal's expected profits are $\Pi_{1}^{P}=e_{1} \theta-w_{1}+\delta\left[p\left(\sqrt{w_{2} \eta \theta} \theta-w_{2}\right)-(1-p) w_{2}\right]$, and outcomes resemble those in the classical reputation literature (see Mailath and Samuelson, 2006). Second, with a separating contract, the first-period effort is higher for a given $w_{2}$ (and determined by a binding (ICR) constraint), however only exerted by the reciprocal type. Then, both types are paid $w_{1}$, whereas the selfish type is fired and only the reciprocal type remains employed in the second period. In this case, the principal's expected profits are $\Pi_{1}^{S}=-w_{1}+p\left[e_{1} \theta+\delta\left(\sqrt{w_{2} \eta \theta} \theta-w_{2}\right)\right]$. If $p$ is sufficiently small, the principal prefers a pooling contract.

This pooling contract, however, relies on the assumption that the reciprocal type cannot reveal himself by choosing a higher effort level. But this restriction generally does not survive the Intuitive Criterion as a refinement of a perfect Bayesian equilibrium (Cho and Kreps, 1987). Assume that, in a pooling contract, an agent chooses an effort level slightly higher than equilibrium effort. Since the selfish type's (IC) constraint binds, whereas the reciprocal type's is slack, a deviation to a higher effort level should indicate that the principal in fact faces the reciprocal type. If the principal responds to this revelation by offering the profit-maximizing second-period wage for the reciprocal type, and if this gives the latter a higher utility than equilibrium play, an upward deviation by the reciprocal type indeed increases his utility. To support the relevance of this argument, in the proof to Proposition 5 I show that for low $p$ and consequently a 
pooling contract, ${ }^{14} e_{1}^{*}=\sqrt[3]{3 \delta p^{2} \eta \theta^{3}}$ and $w_{2}=e_{1}^{3} / 3 \delta=p^{2} \eta \theta^{3}$. If the reciprocal type deviates and chooses an effort level $e_{1}^{*}+\varepsilon$, the principal will take this as a signal that she faces the reciprocal type and might instead offer $w_{2}=\eta \theta^{3} / 4$ (the second-period wage that maximizes her profits with a reciprocal type; see the proof to Lemma 1). This wage also increases the reciprocal type's utility for $p<1 / 2$.

Although a more general characterization of an optimal arrangement under asymmetric information is beyond the scope of this paper, note that a large amount of experimental evidence indicates that cooperation is larger in repeated than in one-shot interactions, even with a predefined last period. This is usually attributed to selfish types imitating those with social preferences to collect future rents (Fehr et al., 2009). I aim to provide support for a complementary story that takes into account that individuals with social preferences also behave strategically. ${ }^{15}$ If the uninformed party can determine the incentive scheme, and in particular ask for a certain effort level, pooling equilibria at which a selfish type imitates a reciprocal type are much harder to maintain. Then, an early separation of types can be achieved by requiring an effort level that just satisfies the reciprocal type's (IC) constraint, with the remaining matches thereafter having a relational contract that produces outcomes resembling my main results (high effort in early periods, declining effort once the last period approaches). Such results have indeed been observed in the lab experiments conducted by Brown et al. (2004). They compare different settings, in particular one in which players (among whom one side assumes the role of firms and the other side represents workers) have the option to form long-term relationships or are randomly matched in each of the 15 rounds. Firms pay wages in every period and ask for effort from "their" workers, who subsequently select their effort levels. Brown et al. (2004) find that effort is significantly larger in the treatment with long-term relationships, where effort only falls in the last two periods. They present a theoretical explanation where some players have fairness preferences and where those without imitate the fair players early on, which mirrors the pooling contract in my setting. However, they observe many separations early on (70 percent in period 1, 65 percent in period 2) but few separations in later periods, which indicates that their outcomes rather resemble separating contracts.

The setting in Brown et al. (2004) admittedly differs from my theoretical model in several ways. For example, students who assume the role of firms might also have social preferences, whereas only the agent is reciprocal in my setting. Nevertheless,

\footnotetext{
${ }^{14}$ More precisely, for $p^{2} \leq\left(\sqrt{\frac{2}{\theta}}\right)^{3} / 3 \delta \eta$.

${ }^{15}$ This is not assumed in most of the reputation literature (Mailath and Samuelson, 2006), where "commitment" types automatically choose cooperative actions.
} 
the presented theoretical analysis, together with a careful analysis of experimental results such as those presented by Brown et al. (2004), justify the notion that not only the "selfish types mimic fair types" story of a pooling contract might contribute to explaining many experimental results. In particular, if players do not face an inflexible environment such as a standard prisoner's dilemma, the possibility of separating types early on and subsequently having a relational contract might also contribute to the high cooperation observed in repeated, but finite, gift-exchange experiments.

\section{Conclusion}

I have shown that the norm of reciprocity can have important implications for the optimal provision of dynamic incentives. Most importantly, formally committing to paying non-discretionary wages in the future can raise a firm's profits because this increases its commitment in the relational contract. Moreover, relational and reciprocity-based incentives reinforce each other and should optimally be used in combination. At the beginning of an employee's career, relational incentives assume a larger role because a longer remaining time horizon increases a firm's commitment. Once the end of the career approaches, reciprocity-based incentives gradually become more important. Finally, more intense competition for employees increases the importance of the norm of reciprocity for the provision of incentives if a lower relationship surplus reduces the effectiveness of relational incentives. 


\section{A. Appendix - Further Extensions}

In the following, I change some of the assumptions made in the main text and explore how this affects my results, using a simplified setting with $T=2$.

\section{A.1. Costs of Commitment}

First, I show that commitment to future wages can still be optimal if it is costly. As in Section 4, I assume that, at the beginning of the first period, the principal not only can pay $w_{1}$, but in addition formally commit to an amount $\hat{w}_{2}$ paid at the beginning of the second period. The agent reacts reciprocally towards $\hat{w}_{2}$ in period 2 , when this payment is actually made. I also assume that $\delta$ does not reflect time preferences anymore, but instead the probability with which a continuation of the relationship is still profitable. More precisely, I assume that, at the end of period 1, $\theta$ drops to zero with probabilty $1-\delta$, for example due to demand fluctuations that let the firm's optimal firm size shrink. In this case, $\hat{w}_{2}$ still has to be paid to the agent and the principal has no possibility to avoid its payment. When the second period comes and $\theta$ has not dropped to zero, the principal can further increase the wage by $w_{2}-\hat{w}_{2}$.

Now, the principal's payoff stream from the perspective of the first period amounts to

$$
\Pi_{1}=e_{1} \theta-b_{1}-w_{1}+\delta \pi_{2}-(1-\delta) \hat{w}_{2}
$$

Thus, there is a direct negative effect of $\hat{w}_{2}$ on $\Pi_{1}$. However, a positive $\hat{w}_{2}$ can be optimal from the perspective of period 1 because it continues to relaxe the principal's dynamic enforcement constraint which becomes

$$
-b_{1}+\delta \pi_{2}-(1-\delta) \hat{w}_{2} \geq-\hat{w}_{2}
$$

Lemma 8 Assume the principal can commit to $\hat{w}_{2}$, as specified above.

- If $\eta^{2} \theta^{3}$ is sufficiently large, (DE) holds for first-best effort and wage levels. Then, $\hat{w}_{2}=0$.

- Otherwise, $\hat{w}_{2}>0$ if $\delta$ is sufficiently large.

The proof can be found in Appendix B.

Now, because a positive $\hat{w}_{2}$ entails direct costs, $\hat{w}_{2}=0$ if the (DE) constraint is slack. If (DE) binds, a positive $\hat{w}_{2}$ is optimal if $\delta$ is sufficiently large. For large values of $\delta$ in relation to $\eta$, it is still possible that the desired level of $\hat{w}_{2}$ exceeds the optimal 
spot-contract wage. In this case, the principal is not only willing to incur costs of commitment if $\theta$ drops to zero (which happens with probability $1-\delta$ ), but in addition reduces future profits if $\theta$ remains positive (which happens with probability $\delta$ ).

\section{A.2. Negative Reciprocity}

So far, I have focused on the positive effects of the norm of reciprocity. I have abstracted from any potential "dark side" of reciprocal preferences in the sense that if an agent is granted a lower payment than expected, he wants to actively harm the principal. The potential consequences of negative reciprocity have been explored by, for example, Dufwenberg and Kirchsteiger (2004), Dohmen et al. (2009), and Netzer and Schmutzler (2014). In this section, I introduce negative reciprocity and show that it leads to the same results as in Section 3, even if $\eta$ does not drop to zero after a deviation by the principal. This section therefore also serves as a robustness exercise to show that my results continue to hold if the agent's preferences are unaffected by the principal's behavior.

I use the approach introduced by Hart and Moore (2008), who assume that the terms of a contract provide reference points and determine a party's ex post performance. If someone receives less than he feels entitled to, he shades on performance, thereby causing a deadweight loss that has to be borne by the other party. Such an assumption has received empirical support from Malmendier and Schmidt (2017), who show that individuals exert negative reciprocity upon a potential gift giver if they expected a gift but did not receive one. I adapt the setting of Hart and Moore (2008) to my environment and assume that the relational contract also determines the agent's reference point.

Therefore, the agent feels entitled to the equilibrium bonus $b_{1}^{*}$. If he receives a lower bonus, his period- 1 utility decreases by $\underline{\eta}\left(b_{1}^{*}-b_{1}\right)$, where $\underline{\eta} \geq 0$ and $b_{1}$ is the bonus actually paid by the principal. Moreover, the agent can reduce this utility loss via shading (e.g., by sabotaging the principal), by an amount $\rho$ at the agent's discretion. I assume that the agent still has to be employed by the principal to shade and the principal can fire the agent before making the choice whether to pay the bonus. Hence, she can escape the shading costs $\rho$ but would then also sacrifice potential future profits. ${ }^{16}$

All this implies that the utility stream of the agent, conditional on not being fired, amounts to

$$
\begin{aligned}
U_{1}= & b_{1}+w_{1}-c\left(e_{1}^{*}\right)+\eta w_{1} \theta e_{1}^{*}-\max \left\{\left[\underline{\eta}\left(b_{1}^{*}-b_{1}\right)-\rho\right], 0\right\} \\
& +\delta\left(w_{2}-c\left(e_{2}^{*}\right)+\eta w_{2} \theta e_{2}^{*}\right) .
\end{aligned}
$$

\footnotetext{
${ }^{16}$ Thus, a bonus is still not feasible in the last period of the game.
} 
The principal's payoff stream if she does not fire the agent before paying the bonus amounts to

$$
\begin{gathered}
\Pi_{1}=e_{1} \theta-w_{1}-b_{1}-\rho \\
+\delta\left(e_{2} \theta-w_{2}\right) .
\end{gathered}
$$

Since shading is not costly for the agent, it is optimal to set $\rho=\underline{\eta}\left(b_{1}^{*}-b_{1}\right)$ (for $\left.b_{1} \leq b_{1}^{*}\right)$. Furthermore, the second-period effort and wage equal $w_{2}=\eta \theta^{3} / 4$ and $e_{2}^{*}=$ $\eta \theta^{2} / 2$, respectively; hence, the second-period profits are $\pi_{2}=\eta \theta^{3} / 4$ (see Lemma 1).

The principal faces two decisions. First, which bonus $b_{1} \in\left[0, b_{1}^{*}\right]$ to pay, and second whether to fire the agent. Concerning the first decision, if the principal decides to pay a bonus $b_{1} \leq b_{1}^{*}$ (and not fire the agent), her profits amount to

$$
\begin{aligned}
\Pi_{1}= & e_{1} \theta-w_{1}+(\underline{\eta}-1) b_{1}-\underline{\eta} b_{1}^{*} \\
& +\delta \frac{\eta \theta^{3}}{4} .
\end{aligned}
$$

This immediately reveals that $b_{1}=0$ is optimal for $\underline{\eta}<1$, whereas $b_{1}=b_{1}^{*}$ for $\underline{\eta} \geq 1$. $b_{1}=b_{1}^{*}$ on the equilibrium path. Thus, $\underline{\eta}<1$ also implies $b_{1}^{*}=b_{1}=0$, and only reciprocity spot contracts are feasible in this case.

Now assume $\underline{\eta} \geq 1$. Then, the principal sets $b_{1}=b_{1}^{*}$ if she does not fire the agent. She will terminate the relationship, however, if the bonus is larger than the period-2 profits, i.e., if $b_{1}^{*}>\delta \pi_{2}$.

The principal's optimization problem becomes maximizing $\pi_{1}=e_{1}^{*} \theta-b_{1}^{*}-w_{1}$, subject to the agent's binding (IC) constraint, which yields $b_{1}^{*}=\left(e_{1}^{*}\right)^{3} / 3-\eta w_{1} e_{1}^{*} \theta+$ $2 / 3\left(\sqrt{\eta w_{1} \theta}\right)^{3}$, as well as subject to $b_{1}^{*} \leq \delta \pi_{2}$. The last condition is equivalent to the (DE) constraint, and thus the problem in this section is the same as the optimization problem in Section 3.

These results are collected in Lemma 9.

Lemma 9 The profit-maximizing equilibrium with negative reciprocity, and a constant norm function $\eta_{t}\left(h^{t-1}\right)=\eta \forall h^{t-1}$, has the following characteristics:

- If $\underline{\eta}<1, b_{1}^{*}=0$. Moreover, $e_{1}^{*}=e_{2}^{*}=\eta \theta^{2} / 2$ and $w_{1}=w_{2}=\eta \theta^{3} / 4$.

- If $\eta \geq 1, b_{1}^{*}>0$, and outcomes are as characterized in Section 3.2, with $w_{1}<$ $w_{2}=\eta \theta^{3} / 4$ and $e_{1}^{*}>e_{2}^{*}=\eta \theta^{2} / 2$, as well as $d e_{2}^{*} / d \eta>d e_{1}^{*} / d \eta$.

If the principal was able to commit to future wages and not to fire the agent (as in Section 4, the agent's off-path shading would hurt the principal even more. Thus, she would also be able to credibly promise a higher bonus in period 1 . 


\section{A.3. Adjustment of the Reference Wage}

Some evidence points toward a declining effect of gifts in long-term interactions. Gneezy and List (2006) conduct a field experiment in which they permanently increase the wages of recruited workers. Although workers respond with an immediate effort increase, this is only temporary, and effort falls to an amount only slightly above the initial level. Jayaraman et al. (2016) explore the effects of a mandated 30\% wage increase for tea pluckers in India. They find that productivity substantially increases immediately after the wage raise. However, it starts falling again in the second month after the change and returns to its initial level after four months. Sliwka and Werner (2017) examine how reciprocal effort is affected by the timing of wage increases. They find that a permanent wage raise only temporarily increases effort and that the only way to permanently benefit from an individual's reciprocal behavior is to constantly raise wages.

This evidence suggests that individuals adapt to wage increases and update their reference wages. In the following, I incorporate this evidence and assume that the reference wage above which the agent is willing to reciprocate depends on his past wages. More precisely, the agent starts with a reference wage of zero. In the second period, the first-period wage $w_{1}$ becomes the new reference wage. ${ }^{17}$ Hence, the agent's utilities are

$$
\begin{aligned}
& u_{1}=b_{1}+w_{1}-c\left(e_{1}^{*}\right)+\eta w_{1} e_{1}^{*} \theta \\
& u_{2}=b_{2}+w_{2}-c\left(e_{2}^{*}\right)+\max \left\{0, \eta\left(w_{2}-w_{1}\right) e_{2}^{*} \theta\right\} .
\end{aligned}
$$

First, I compute the profit-maximizing spot reciprocity contract in the last period. Then, no bonus is paid, and - taking into account that setting $w_{2} \geq w_{1}$ is optimal effort maximizes $-e_{2}^{3} / 3+\eta\left(w_{2}-w_{1}\right) e_{2} \theta$. As shown in Lemma 7, effort is unaffected by the higher reference wage; hence, $e_{2}^{*}=\eta \theta^{2} / 2$ and $w_{2}^{*}=\eta \theta^{3} / 4+w_{1}$.

The outcomes for an optimal relational contract are given in Lemma A.3.

Lemma 10 Assume the second-period reference wage is equal to $w_{1}$. Then, $w_{1}<w_{2}$. Moreover, the (DE) constraint might or might not bind.

- If it does not bind, $d e_{1}^{*} / d \eta<d e_{2}^{*} / d \eta$. Furthermore, there exists a $\bar{\eta}>0$ such that the optimal wage is zero for $\eta \leq \bar{\eta}$. In this case, $e_{1}^{*}>e_{2}^{*}$. For $\eta>\bar{\eta}$, setting a strictly positive wage is optimal, and $e_{1}^{*}$ can be smaller or larger than $e_{2}^{*}$.

\footnotetext{
${ }^{17}$ This resembles the setting of DellaVigna et al. (2017), who apply a similar assumption (with the exception that the reference point path is exogenous, whereas it is endogenous in my setting) to a model of reference dependent loss aversion.
} 
- If it binds, there exists a $\tilde{\eta}>0$ such that the optimal wage equals zero for $\eta \leq \tilde{\eta}$, whereas it is strictly positive for $\eta \geq \tilde{\eta}$. In both cases, $e_{1}^{*}$ can be smaller or larger than $e_{2}^{*}$.

$\bar{\eta}$ can be smaller or larger than $\tilde{\eta}$, and both are larger than if the second-period reference wage equals zero independent of $w_{1}$.

The proof can be found in Appendix B.

The principal is reluctant to trigger the agent's reciprocal preferences in the first period. In particular, if $\delta$ is large, she wants to maintain this opportunity until later when relational contracts are no longer feasible. Therefore, the threshold for $\eta$ above which a positive first-period wage is paid is larger than that in Section 3 - implying that the backloading of reciprocity-based incentives is more pronounced than with a constant reference wage. A higher $w_{1}$ also does not necessarily relax the (DE) constraint anymore (which implies that $\tilde{\eta}$ does not have to be smaller than $\bar{\eta}$ ). This is because a positive first-period wage has two effects on the tightness of the (DE) constraint. On the one hand, the necessary bonus to implement a certain effort level is reduced, which relaxes the constraint. On the other hand, future profits are reduced via the adjustment of the reference wage, which tightens the constraint. Moreover, $e_{1}^{*}$ is not necessarily larger than $e_{2}^{*}$ because the reluctance to pay a positive $w_{1}$ also reduces the agent's willingness to exert effort in the first period.

\section{A.4. Reciprocity Triggered by Rents}

Finally, I explore the implications of reciprocity being triggered by the agent's material rent, in contrast to only by monetary payments. Thus, I assume that the agent's perperiod utilities are

$$
\begin{aligned}
& u_{1}=\left(b_{1}+w_{1}-c\left(e_{1}\right)\right)\left(1+\eta e_{1} \theta\right) \\
& u_{2}=\left(w_{2}-c\left(e_{2}\right)\right)\left(1+\eta e_{2} \theta\right) .
\end{aligned}
$$

Importantly, when choosing his effort level, the agent also reciprocates on the equilibrium bonus of this period before it is paid. Hence, the principal is less inclined to pay a positive fixed wage in the first period. Only if a sufficiently tight (DE) constraint considerably restrains the bonus is $w_{1}$ positive.

Formally, effort in the second period is given by the agent's first order condition,

$$
-e_{2}^{2}-\frac{4}{3} e_{2}^{3} \eta \theta+w_{2} \eta \theta=0
$$


This is taken into account by the principal who sets $w_{2}$ to maximize $\pi_{2}=e_{2} \theta-w_{2}$.

In the first period, the principal's (DE) constraint still equals $-b_{1}+\delta \pi_{2} \geq 0$, whereas the agent's (IC) constraint becomes

$$
\left(b_{1}+w_{1}-\frac{\left(e_{1}^{*}\right)^{3}}{3}\right)\left(1+\eta e_{1}^{*} \theta\right) \geq\left(w_{1}-\frac{\left(\tilde{e}_{1}\right)^{3}}{3}\right)\left(1+\eta \tilde{e}_{1} \theta\right) .
$$

Here, $\tilde{e}_{1}$ is characterized by $-\tilde{e}_{1}^{2}-\frac{4}{3} \tilde{e}_{1}^{3} \eta \theta+w_{1} \eta \theta=0$, and $e_{1}^{*}>\tilde{e}_{1}$ if $b_{1}>0$.

Lemma 11 Assume that the agent's preferences for reciprocity are triggered by his material rent. Then, the $(D E)$ constraint binds given $T=2$ and $\delta \leq 1$. Moreover, there exists a $\tilde{\eta}>0$ such that the optimal wage equals zero for $\eta \leq \tilde{\eta}$, whereas it is strictly positive for $\eta \geq \tilde{\eta}$.

In any case, $e_{1}^{*}>e_{2}^{*}$ and $w_{1}<w_{2}$.

The proof can be found in Appendix B.

With $T=2$, second-period profits cannot be sufficiently large for a non-binding (DE) constraint given $\delta \leq 1$. However, in a more general setting with more than two periods, (DE) might indeed be slack. In this case, the proof to Lemma 11 reveals that paying a positive wage would not be optimal because the purpose of a positive wage - triggering the agent's reciprocal inclinations - can equivalently be achieved by a bonus, which additionally allows for higher effort via the use of relational incentives. With a binding (DE) constraint, the principal might pay a fixed wage in the first period, but only if $\eta$ is large enough. Finally, it can be shown that allowing the principal to commit to nondiscretionary future wages as in Section 4 also relaxes the (DE) constraint if reciprocity is triggered by the material rent the agent receives.

\section{B. Appendix - Omitted Proofs}

Proof of Lemma 1 I maximize profits $\pi=e \theta-w$, taking into account that effort equals $e=\sqrt{\eta w \theta}$, and that the agent's (IR) constraint, $u=w-e^{3} / 3+\eta w e \theta=w+$ $(2 / 3) \sqrt{\eta w}^{3} \geq 0$, must be satisfied. Naturally, the latter holds for any $w \geq 0$.

The principal's first order condition equals

$$
\frac{d \pi}{d w}=\frac{d e}{d w} \theta-1=0
$$

which yields 


$$
w=\frac{\eta \theta^{3}}{4}
$$

Hence,

$$
e^{*}=\frac{\eta \theta^{2}}{2}
$$

and $\pi=\eta \theta^{3} / 4, u=\eta \theta^{3} / 4+\eta^{3} \theta^{6} / 12>0$.

Proof of Lemma 2 The principal maximizes $\Pi_{1}$, subject to (IC) and (DE) constraints for every period,

$$
\begin{aligned}
b_{t}-\frac{\left(e_{t}^{*}\right)^{3}}{3}+\eta w_{t}^{n d} e_{t}^{*} \theta+\delta U_{t+1} \geq-\frac{\left(\tilde{e}_{t}\right)^{3}}{3}+\eta w_{t}^{n d} \tilde{e}_{t} \theta+\delta \tilde{U}_{t+1} \\
-b_{t}+\delta \Pi_{t+1} \geq 0
\end{aligned}
$$

$\tilde{U}_{t+1}$ describes the agent's off-path continuation utility after a deviation by himself. After the principal deviates (downwards), $\eta$ drops to zero, and continuation payoffs of principal and agent are zero. For this proof, I focus on deviations by the agent, hence assume (DE) constraints hold on and off the equilibrium path and $\eta$ remains constant. I only have to take care of the possibility of upward deviations by the principal (i.e., increasing future payments of $w_{t}^{\text {nd }}$ after a deviation by the agent), which do not reduce $\eta$. If these upward deviations are optimal off the equilibrium path, they affect the size of $\tilde{U}_{t}$ and hence the agent's incentives to provide equilibrium effort.

First, it is optimal without loss of generality to set $w_{t}^{d}=0$ after all histories: Assume $w_{t}^{d}>0$. Reducing it to zero and increasing $b_{t-1}$ by $\delta w_{t}^{d}$ leaves all payoffs and constraints unaffected. In the following, I hence assume that $w_{t}^{d}=0$ in all periods $t$ and for all histories.

Second, $w_{t}^{n d}$ is - by definition - independent of the agent's past effort choices. Thus, I need to prove consistency in the sense that it is indeed optimal for the principal to not change $w_{t}^{n d}$ after a deviation by the agent. In this respect, I assume that all $w_{t}^{\text {nd }}$ in a profit-maximizing relational contract are smaller than the wage in a reciprocity spot contract (Lemma 1) and later verify that this is indeed optimal (see Proposition 1). Then, firing the agent after he did not perform accordingly is not subgame perfect and can thus be ruled out as a potential response of the principal. The reason is that, with non-discretionary wages not exceeding the profit-maximizing wage in a reciprocity spot contract, the principal makes positive profits even if no relational incentives are provided on top (if some $w_{t}^{\text {nd }}$ were larger, I would have to check whether spot contracts with "too high" wages still generate positive profits). This also implies that the principal 
does not lower non-discretionary wages after a deviation by the agent.

Third, I need to verify that increasing $w^{\text {nd }}$ after a deviation by the agent is not optimal (otherwise, a deviation might increase the agent's continuation utility and consequently his incentives to deviate). But this follows from the definition of $w_{t}^{\text {nd }}$, which states that the agent only reciprocates to wage components that are independent of the agent's past effort, hence increasing the wage after a deviation of the agent would not induce a stronger reciprocal reaction.

All this implies that $\tilde{U}_{t}$ contains the same non-discretionary wage stream $\left\{w_{\tau}^{n d}\right\}_{\tau=t}^{T}$ as $U_{t}$. Moreover, I can set $\tilde{U}_{t}=\sum_{\tau=t}^{T} \delta^{\tau-t}\left(w_{\tau}^{n d}-\frac{\tilde{e}_{\tau}^{3}}{3}+\eta w_{\tau}^{n d} \tilde{e}_{\tau} \theta\right)$, which corresponds to the agent's minmax payoff given $\left\{w_{\tau}^{n d}\right\}_{\tau=t}^{T}$ (and provided $w_{t} \geq 0$, which rules out negative upfront payments to extract the agent's "reciprocity rent). The reason is that, using standard arguments, a series of spot contracts always constitutes an equilibrium of such a finitely repeated game.

Fourth, I show that for given values $w_{t}^{\text {nd }}$ and $e_{t}$, it is (weakly) optimal for the principal to set $b_{t}-\frac{e_{t}^{3}}{3}+\eta w_{t}^{n d} e_{t} \theta=-\frac{\tilde{e}_{t}^{3}}{3}+\eta w_{t}^{n d} \tilde{e}_{t} \theta$ in all periods. To do so, I proceed sequentially and start with period $t=1$ :

- Assume $b_{1}-\frac{e_{1}^{3}}{3}+\eta w_{1}^{n d} e_{1} \theta>-\frac{\tilde{e}_{1}^{3}}{3}+\eta w_{1}^{n d} \tilde{e}_{1} \theta$. Reduce $b_{1}$ by a small $\varepsilon>0$, which increases $\Pi_{1}$ and relaxes the first-period (DE) constraint.

- Assume $b_{1}-\frac{e_{1}^{3}}{3}+\eta w_{1}^{n d} e_{1} \theta<-\frac{\tilde{e}_{1}^{3}}{3}+\eta w_{1}^{n d} \tilde{e}_{1} \theta$. Because $w^{d}=0 \forall t$, the (IC) constraint for period $t=1$ requires at least one period $\tau>1$ in which $b_{\tau}$ $\frac{e_{\tau}^{3}}{3}+\eta w_{\tau}^{n d} e_{\tau} \theta>-\frac{\tilde{e}_{\tau}^{3}}{3}+\eta w_{\tau}^{n d} \tilde{e}_{\tau} \theta$. Assume $\tau_{1}$ is the first of these periods. Reduce $b_{\tau_{1}}$ by $\varepsilon>0$ and increase $b_{1}$ by $\delta^{\tau_{1}-1} \varepsilon$. Proceed until either $b_{1}-\frac{e_{1}^{3}}{3}+$ $\eta w_{1}^{n d} e_{1} \theta=-\frac{\tilde{e}_{1}^{3}}{3}+\eta w_{1}^{n d} \tilde{e}_{1} \theta$ or $b_{\tau_{1}}-\frac{e_{\tau_{1}}^{3}}{3}+\eta w_{\tau_{1}}^{n d} e_{\tau_{1}} \theta=-\frac{\tilde{e}_{\tau_{1}}^{3}}{3}+\eta w_{\tau_{1}}^{n d} \tilde{e}_{\tau_{1}} \theta$. In the latter case, move to the second period $\tau_{2}>\tau_{1}$ in which $b_{\tau_{2}}-\frac{e_{\tau_{2}}^{3}}{3}+\eta w_{\tau_{2}}^{n d} e_{\tau_{2}} \theta>$ $-\frac{\tilde{e}_{\tau_{2}}^{3}}{3}+\eta w_{\tau_{2}}^{n d} \tilde{e}_{\tau_{2}} \theta$ (such a period must exist as long as $b_{1}-\frac{e_{1}^{3}}{3}+\eta w_{1}^{n d} e_{1} \theta<$ $\left.-\frac{\tilde{e}_{1}^{3}}{3}+\eta w_{1}^{n d} \tilde{e}_{1} \theta\right)$, reduce $b_{\tau_{2}}$ by $\varepsilon>0$ and increase $b_{1}$ by $\delta^{\tau_{2}-1} \varepsilon$, and so on. Continue until $b_{1}-\frac{e_{1}^{3}}{3}+\eta w_{1}^{n d} e_{1} \theta=-\frac{\tilde{e}_{1}^{3}}{3}+\eta w_{1}^{n d} \tilde{e}_{1} \theta$.

In period $t=2$, proceed accordingly if $b_{2}-\frac{e_{2}^{3}}{3}+\eta w_{2}^{n d} e_{2} \theta \neq 0$, as well as in all following periods.

It follows that $u_{t}=w_{t}^{n d}+b_{t}-\frac{e_{t}^{3}}{3}+\eta w_{t}^{n d} e_{1} \theta=w_{t}^{n d}-\frac{\tilde{e}_{t}^{3}}{3}+\eta w_{t}^{n d} \tilde{e}_{t} \theta$, and consequently that $U_{t}=\tilde{U}_{t}$ in all periods $t$, and that all (IC) constraints hold as equalities.

Finally, these results imply that there exists a sequentially optimal profit-maximizing equilibrium, in the sense that maximizing $\Pi_{1}$ is equivalent to maximizing each perperiod profit $\pi_{t}$, subject to (DE) and binding (IC) constraints. This is because the agent's 
incentives to exert effort in any period $t$ are solely determined by payments made in period $t, w_{t}^{n d}$ and $b_{t}$. There, $b_{t}$ is bounded by the principal's future payoff stream, thus maximizing each $\pi_{t}$ ceteris paribus maximizes $\Pi_{1}$, but also yields the largest maximum feasible value of $b_{t}$ without adverse effect on (IC) constraints.

Proof of Lemma 3 If the (DE) constraint does not bind in a period $t$, the principal maximizes profits $\pi_{t}=e_{t} \theta-\left(\left(e_{t}\right)^{3} / 3-\eta w_{t} e_{t} \theta+2 / 3\left(\sqrt{\eta w_{t} \theta}\right)^{3}\right)-w_{t}$, subject to $w_{t} \geq 0$.

The Lagrange function equals $L_{t}=e_{t} \theta-\left(e_{t}\right)^{3} / 3+\eta w_{t} e_{t} \theta-2 / 3\left(\sqrt{\eta w_{t} \theta}\right)^{3}-w_{t}+$ $\lambda_{w_{t}} w_{t}$, where $\lambda_{w_{t}} \geq 0$ represents the Lagrange parameter for the agent's limited liability constraint, giving first order conditions

$$
\begin{aligned}
& \frac{\partial L_{t}}{\partial e_{t}^{*}}=\theta-\left(e_{t}\right)^{2}+\eta w_{t} \theta=0 \\
& \frac{\partial L_{t}}{\partial w_{t}}=\eta \theta\left(e_{t}-\sqrt{\eta w_{t} \theta}\right)-1+\lambda_{w_{t}}=0 .
\end{aligned}
$$

First, assume that $\lambda_{w_{t}}=0$. Then, the first order conditions yield $w_{t}=\left(\eta^{2} \theta^{3}-1\right)^{2} /\left(4 \eta^{3} \theta^{3}\right)$ and $e_{t}^{*}=\left(1+\eta^{2} \theta^{3}\right) /(2 \eta \theta)$. Second, assume that $\lambda_{w_{t}}>0$ and hence $w_{t}=0$. Then, $e_{t}^{*}=\sqrt{\theta}$ and $\pi_{t}=\frac{2}{3}(\sqrt{\theta})^{3}$. To establish the existence of $\bar{\eta}$, note that $d \pi_{t} /\left.d w_{t}\right|_{w_{t}=0}=$ $\sqrt{\eta^{2} \theta^{3}}-1$. This is positive for $\eta>\sqrt{1 / \theta^{3}}$, hence a strictly positive wage is optimal in this case and not otherwise.

Proof of Lemma 4 Including the respective (DE) constraints, the Lagrange function of the principal's maximization problem in a period $t$ becomes

$$
\begin{aligned}
L_{t} & =e_{t} \theta-e_{t}^{3} / 3+\eta w_{t} e_{t} \theta-2 / 3\left(\sqrt{\eta w_{t} \theta}\right)^{3}-w_{t} \\
& +\lambda_{D E_{t}}\left[\delta \Pi_{t+1}-\frac{2}{3}\left(\sqrt{\eta w_{t} \theta}\right)^{3}-e_{t}^{3} / 3+\eta w_{t} \theta e_{t}\right]+w_{t} \lambda_{w_{t}}
\end{aligned}
$$

where $\lambda_{w_{t}} \geq 0$ represents the Lagrange parameter for the agent's limited liability constraint and $\lambda_{D E_{t}} \geq 0$ represents the Lagrange parameter for the principal's dynamic enforcement constraint. 
First order conditions are

$$
\begin{aligned}
& \frac{\partial L}{\partial e_{t}^{*}}=\theta-e_{t}^{2}+\eta w_{t} \theta+\lambda_{D E_{t}}\left[-e_{t}^{2}+\eta w_{t} \theta\right]=0 \\
& \frac{\partial L}{\partial w_{t}}=\eta \theta e_{t}-\eta \theta \sqrt{\eta w_{t} \theta}-1+\lambda_{D E_{t}}\left[-\eta \theta \sqrt{\eta w_{t} \theta}+\eta \theta e_{t}\right]+\lambda_{w_{t}}=0 .
\end{aligned}
$$

First, assume that $\lambda_{w_{t}}=0$. Then, the first order conditions yield $w_{t}=\frac{\left(\eta^{2} \theta^{3}\left(1+\lambda_{D E_{t}}\right)-1\right)^{2}}{4 \eta^{3} \theta^{3}\left(1+\lambda_{D E_{t}}\right)^{2}}$ and $e_{t}^{*}=\frac{1+\eta^{2} \theta^{3}\left(1+\lambda_{D E_{t}}\right)}{2 \eta \theta\left(1+\lambda_{D E_{t}}\right)}$. It follows that, given $\lambda_{D E_{t}}>0$ and $\eta>\bar{\eta}$ (i.e., $\eta^{2} \theta^{3}-$ 1 , implying that $w_{t}>0$ if (DE) does not bind), $\frac{\left(\eta^{2} \theta^{3}\left(1+\lambda_{D E_{t}}\right)-1\right)^{2}}{4 \eta^{3} \theta^{3}\left(1+\lambda_{D E_{t}}\right)^{2}}>\frac{\left(\eta^{2} \theta^{3}-1\right)^{2}}{4 \eta^{3} \theta^{3}}$ and $\frac{1+\eta^{2} \theta^{3}\left(1+\lambda_{D E_{t}}\right)}{2 \eta \theta\left(1+\lambda_{D E_{t}}\right)}<\frac{1+\eta^{2} \theta^{3}}{2 \eta \theta}$.

Second, assume that $\lambda_{w_{t}}>0$ and hence $w_{t}=0$. Then, $e_{t}^{*}=\sqrt{\theta /\left(1+\lambda_{D E_{t}}\right)}$. To establish the existence of $\tilde{\eta}$, note that $\partial L /\left.\partial w_{t}\right|_{w_{t}=0}=\sqrt{\eta^{2} \theta^{3}\left(1+\lambda_{D E_{t}}\right)}-1=0$. This is positive for $\eta>\sqrt{1 / \theta^{3}\left(1+\lambda_{D E_{t}}\right)}$, and thus a strictly positive wage is optimal in this case and not otherwise. Finally, for $\lambda_{D E_{t}}>0, \tilde{\eta}=\sqrt{1 / \theta^{3}\left(1+\lambda_{D E_{t}}\right)}<\bar{\eta}=\sqrt{1 / \theta^{3}}$. Moreover, $\tilde{\eta}$ increases in $\delta$ because $\lambda_{D E_{t}}$ decreases in $\delta$ (see the proof to Lemma 5).

Proof of Lemma 5 The (DE) constraint in period $T-1$ (where on-path continuation profits are $\left.\Pi_{T}=\eta \theta^{3} / 4\right)$ equals $\left(e_{t}^{*}\right)^{3} / 3-\eta w_{t} \theta e_{t}^{*} \leq \delta \eta \theta^{3} / 4-2 / 3\left(\sqrt{\eta w_{t} \theta}\right)^{3}$. First, note that for $\eta \leq \bar{\eta}$, in which case the first-best wage is zero and first-best effort equals $\sqrt{\theta}$, the (DE) constraint equals $(\sqrt{\theta})^{3} / 3 \leq \delta \eta \theta^{3} / 4$. This cannot hold if $\eta \leq \bar{\eta}=$ $\sqrt{1 / \theta^{3}}$, even for $\delta \rightarrow 1$.

Therefore, assume $\eta>\bar{\eta}$ for the remainder of this proof. Then, first-best effort and wage levels are $e=\left(1+\eta^{2} \theta^{3}\right) /(2 \eta \theta)$ and $w=\left(\eta^{2} \theta^{3}-1\right)^{2}\left(4 \eta^{3} \theta^{3}\right)$, and the (DE) constraint in period $T-1$ becomes

$$
\frac{3 \eta^{2} \theta^{3}-1}{6 \eta^{3} \theta^{3}} \leq \delta \frac{\eta \theta^{3}}{4}
$$

Because $\eta>\bar{\eta}$, the left-hand-side is strictly positive. Therefore, the constraint is violated for first-best effort and wage levels if $\delta \rightarrow 0$.

To show that first-best effort can be implemented in period $T-1$ if $\eta$ is sufficiently large, I compute the derivative of the left-hand-side of 1 and obtain $\left(1-\eta^{2} \theta^{3}\right) / 2 \eta^{4} \theta^{3}$, which is negative for $\eta>\bar{\eta}$. Moreover, $\lim _{\eta \rightarrow \infty} \frac{3 \eta^{2} \theta^{3}-1}{6 \eta^{3} \theta^{3}}=0$, whereas the right-hand side of 1 is strictly positive and increasing in $\eta$. Therefore, 1 is satisfied if $\eta$ is sufficienty large.

Concerning the second part of the Lemma, recall the (DE) constraint for period $t$ 
equals $\left(e_{t}^{*}\right)^{3} / 3-\eta w_{t} \theta e_{t}^{*} \leq \delta \Pi_{t+1}-\frac{2}{3}\left(\sqrt{\eta w_{t} \theta}\right)^{3}$. It follows that, for a given $w_{t}$, the maximum implementable effort in period $t$ is ceteris paribus strictly increasing in $\Pi_{t+1}$, whereas per-period profits $\pi_{t}$ are consequently weakly increasing in $\Pi_{t+1}$. Furthermore, per-period profits in periods $t<T$ can be expressed as functions of $\Pi_{t+1}$, i.e. $\pi_{t}\left(\Pi_{t+1}\right)$, with $\pi_{t}^{\prime} \geq 0$.

The profit-maximizing spot reciprocity contract is the principal's optimal choice in the last period $T$, hence $\pi_{T}=\Pi_{T}=\eta \theta^{3} / 4$. In all previous periods, the principal still has the option to implement the spot reciprocity contract (by setting $b_{t}^{*}=0$ and $w_{t}=$ $\left.\eta \theta^{3} / 4\right)$, therefore $\pi_{t} \geq \pi_{T} \forall t$.

Now, I apply proof by induction to verify that $\Pi_{t-1}>\Pi_{t}$. First, $\Pi_{T-1}>\Pi_{T}$ because

$$
\Pi_{T-1}=\pi_{T-1}+\delta \Pi_{T} \geq \pi_{T}+\delta \Pi_{T}=\Pi_{T}(1+\delta)>\Pi_{T}
$$

For the induction step, assume that $\Pi_{t}>\Pi_{t+1}$. Since $\pi_{t}^{\prime}\left(\Pi_{t+1}\right) \geq 0, \pi_{t-1} \geq \pi_{t}$. Therefore, $\Pi_{t-1}=\pi_{t-1}+\delta \Pi_{t} \geq \pi_{t}+\delta \Pi_{t}>\pi_{t}+\delta \Pi_{t+1}=\Pi_{t}$, which completes the proof.

Proof of Proposition 1 First, assume $\eta>\bar{\eta}=\sqrt{1 / \theta^{3}}$, hence $w_{t}>0 \forall t$. Furthermore, in Lemmas 3 and 4, I have established that $w_{t}=\frac{\left(\eta^{2} \theta^{3}\left(1+\lambda_{D E_{t}}\right)-1\right)^{2}}{4 \eta^{3} \theta^{3}\left(1+\lambda_{D E_{t}}\right)^{2}}$ and $e_{t}^{*}=$ $\frac{1+\eta^{2} \theta^{3}\left(1+\lambda_{D E_{t}}\right)}{2 \eta \theta\left(1+\lambda_{D E_{t}}\right)}$, where $\lambda_{D E_{t}}$ is the Lagrange parameter associated with the (DE) constraint in period $t$. Hence, $w_{t}=w_{t-1}$ and $e_{t}^{*}=e_{t-1}$ if $\lambda_{D E_{t}}=\lambda_{D E_{t-1}}=0$. By Lemma 4, if $\lambda_{D E_{t-1}}=0$ but $\lambda_{D E_{t}}>0$, then $w_{t}>w_{t-1}$ and $e_{t}^{*}<e_{t-1}$. Finally, assume that $\lambda_{D E_{t-1}}>0$. First, I show that in this case also $\lambda_{D E_{t}}>0$ : Plugging $w_{t-1}=\frac{\left(\eta^{2} \theta^{3}\left(1+\lambda_{D E_{t-1}}\right)-1\right)^{2}}{4 \eta^{3} \theta^{3}\left(1+\lambda_{D E_{t-1}}\right)^{2}}$ and $e_{t-1}^{*}=\frac{1+\eta^{2} \theta^{3}\left(1+\lambda_{D E_{t-1}}\right)}{2 \eta \theta\left(1+\lambda_{D E_{t-1}}\right)}$ into the binding (DE) constraint for period $t-1$ yields

$$
\frac{3 \eta^{2} \theta^{3}\left(1+\lambda_{D E_{t-1}}\right)-1}{6 \eta^{3} \theta^{3}\left(1+\lambda_{D E_{t-1}}\right)^{3}}=\delta \Pi_{t} .
$$

I can also treat $\lambda_{D E_{t-1}}$ as a function of $\Pi_{t}$. By the implicit function theorem, $\frac{\partial \lambda_{D E_{t-1}}}{\partial \Pi_{t}}=$ $\frac{2 \delta \eta^{3} \theta^{3}\left(1+\lambda_{D E_{t-1}}\right)^{4}}{1-2 \eta^{2} \theta^{3}\left(1+\lambda_{D E_{t-1}}\right)}<0\left(\right.$ since $\eta>\bar{\eta}$ implies $\left.\eta^{2} \theta^{3}>1\right)$. Hence, Lemma 5 yields $\lambda_{D E_{t-1}}<$ $\lambda_{D E_{t}}$, which implies $\lambda_{D E_{t-1}}>0 \Rightarrow \lambda_{D E_{t}}>0$. Furthermore, if $\lambda_{D E_{t}}=0$ in a period $t$, this also holds for all previous periods.

The wage schedule is increasing in periods $t<T$ since $\frac{\partial w_{t}}{\partial \lambda_{D E_{t}}}=\frac{\left(\eta^{2} \theta^{3}\left(1+\lambda_{D E_{t}}\right)-1\right)}{2 \eta^{3} \theta^{3}\left(1+\lambda_{D E_{t}}\right)^{3}}>0$, whereas the effort path is decreasing because of $\frac{\partial e_{t}^{*}}{\partial \lambda_{D E_{t}}}=\frac{-1}{2 \eta \theta\left(1+\lambda_{D E_{t}}\right)^{2}}<0$. Finally, 
wage and effort in period $T$ are $e_{T}^{*}=\frac{\eta \theta^{2}}{2}$ and $w_{T}=\frac{\eta \theta^{3}}{4}$, respectively. $e_{T}^{*}<e_{t}^{*}$ for all $t<T$ follows from $\frac{\eta \theta^{2}}{2}<\frac{1+\eta^{2} \theta^{3}\left(1+\lambda_{D E_{t}}\right)}{2 \eta \theta\left(1+\lambda_{D E_{t}}\right)}\left(\Leftrightarrow \eta^{2} \theta^{3}\left(1+\lambda_{D E_{t}}\right)<1+\eta^{2} \theta^{3}\left(1+\lambda_{D E_{t}}\right)\right)$. $w_{T}>w_{t}$ for all $t<T$ follows from $\frac{\eta \theta^{3}}{4}>\frac{\left(\eta^{2} \theta^{3}\left(1+\lambda_{D E_{t}}\right)-1\right)^{2}}{4 \eta^{3} \theta^{3}\left(1+\lambda_{D E_{t}}\right)^{2}}\left(\Leftrightarrow 2 \eta^{2} \theta^{3}\left(1+\lambda_{D E_{t}}\right)>1\right)$.

For the remainder of the proof, assume $\eta \leq \bar{\eta}$, hence $w_{t}=0$ and $e_{t}^{*}=\sqrt{\theta}$ if $\lambda_{D E_{t}}=0$. As before, $\lambda_{D E_{t}}=0$ implies $\lambda_{D E_{t-1}}=0$, and $\lambda_{D E_{t}}>0$ implies $\lambda_{D E_{t+1}}>\lambda_{D E_{t}}$.

The following cases still have to be explored:

- $\lambda_{D E_{t}}>0$ and $w_{t}>0$. Then, $w_{t}=\frac{\left(\eta^{2} \theta^{3}\left(1+\lambda_{D E_{t}}\right)-1\right)^{2}}{4 \eta^{3} \theta^{3}\left(1+\lambda_{D E_{t}}\right)^{2}}$ and $e_{t}^{*}=\frac{1+\eta^{2} \theta^{3}\left(1+\lambda_{D E_{t}}\right)}{2 \eta \theta\left(1+\lambda_{D E_{t}}\right)}$, and the previous analysis regarding wages $w_{\tau}$ and effort levels $e_{\tau}$, for $\tau>t$, can be applied. The previous analysis can also be applied if $\lambda_{D E_{t-1}}>0$ and $w_{t-1}>0$.

Now, assume $\lambda_{D E_{t-1}}>0$ and $w_{t-1}=0$. Then, $e_{t-1}=\sqrt{\theta /\left(1+\lambda_{D E_{t-1}}\right)}$ (see the proof to Lemma 4), and I have to show that

$$
\sqrt{\frac{\theta}{\left(1+\lambda_{D E_{t-1}}\right)}}>\frac{1+\eta^{2} \theta^{3}\left(1+\lambda_{D E_{t}}\right)}{2 \eta \theta\left(1+\lambda_{D E_{t}}\right)} .
$$

In the proof to Lemma 4, I haven proven that $w_{t-1}=0$ implies $\eta \leq \sqrt{1 / \theta^{3}\left(1+\lambda_{D E_{t-1}}\right)}$, which can be re-written to $\sqrt{\theta /\left(1+\lambda_{D E_{t-1}}\right)} \geq \eta \theta^{2}$. Therefore, it is sufficient to show that $\eta \theta^{2}>\left[1+\eta^{2} \theta^{3}\left(1+\lambda_{D E_{t}}\right)\right] /\left[2 \eta \theta\left(1+\lambda_{D E_{t}}\right)\right]$, which becomes $\eta>$ $\sqrt{1 / \theta^{3}\left(1+\lambda_{D E_{t}}\right)}$. This, however, is implied by $w_{t}>0$ (see the proof to Lemma 4).

- $\lambda_{D E_{t}}=0$ and $\lambda_{D E_{t+1}}>0$, with $w_{t+1}=0$. Now, $e_{t+1}^{*}<e_{t}^{*}$ follows from $e_{t}^{*}=$ $\sqrt{\theta /\left(1+\lambda_{D E_{t}}\right)}, e_{t+1}^{*}=\sqrt{\theta /\left(1+\lambda_{D E_{t+1}}\right)}$ and $\lambda_{D E_{t+1}}>\lambda_{D E_{t}}$.

- $\lambda_{D E_{t}}=0$ and $\lambda_{D E_{t+1}}>0$, with $w_{t+1}>0$. Now, $e_{t}^{*}=\sqrt{\theta}$ and $e_{t+1}^{*}=\frac{1+\eta^{2} \theta^{3}\left(1+\lambda_{D E_{t+1}}\right)}{2 \eta \theta\left(1+\lambda_{D E_{t+1}}\right)}$, and I have to show that

$$
\begin{gathered}
\sqrt{\theta}>\frac{1+\eta^{2} \theta^{3}\left(1+\lambda_{D E_{t+1}}\right)}{2 \eta \theta\left(1+\lambda_{D E_{t+1}}\right)} \\
\Leftrightarrow\left(1+\lambda_{D E_{t+1}}\right)\left(2 \sqrt{\eta^{2} \theta^{3}}-\eta^{2} \theta^{3}\right)>1 .
\end{gathered}
$$

Again, $w_{t+1}>0$ implies $\left(1+\lambda_{D E_{t+1}}\right)>1 / \eta^{2} \theta^{3}$ (see the proof to Lemma 4), hence it is sufficient to prove that (taking into acount that $\eta \leq \bar{\eta}$ implies $2 \sqrt{\eta^{2} \theta^{3}}-$ 


$$
\left.\eta^{2} \theta^{3}>0\right)
$$

$$
\begin{gathered}
\frac{\left(2 \sqrt{\eta^{2} \theta^{3}}-\eta^{2} \theta^{3}\right)}{\eta^{2} \theta^{3}} \geq 1 \\
\Leftrightarrow 2 \sqrt{\eta^{2} \theta^{3}}\left(1-\sqrt{\eta^{2} \theta^{3}}\right) \geq 0,
\end{gathered}
$$

which holds because of $\eta \leq \bar{\eta}$.

Concerning the bonus, note that the binding (IC) constraint delivers $b_{t}=\left(e_{t}^{*}\right)^{3} / 3-$ $\eta w_{t} e_{t}^{*} \theta+2 / 3\left(\sqrt{\eta w_{t} \theta}\right)^{3}$. It follows that, if $w_{t}=\frac{\left(\eta^{2} \theta^{3}\left(1+\lambda_{D E_{t}}\right)-1\right)^{2}}{4 \eta^{3} \theta^{3}\left(1+\lambda_{D E_{t}}\right)^{2}}>0$

$$
b_{t}=\frac{3 \eta^{2} \theta^{3}\left(1+\lambda_{D E_{t}}\right)-1}{6 \eta^{3} \theta^{3}\left(1+\lambda_{D E_{t}}\right)^{3}}
$$

with $\frac{d b_{t}}{\left(1+\lambda_{D E_{t}}\right)}=\frac{-2 \eta^{2} \theta^{3}\left(1+\lambda_{D E_{t}}\right)+1}{2 \eta^{3} \theta^{3}\left(1+\lambda_{D E_{t}}\right)^{4}}<0$.

Moreover, if $w_{t}=0$, then

$$
b_{t}=\frac{\left(\sqrt{\frac{\theta}{\left(1+\lambda_{D E_{t}}\right)}}\right)^{3}}{3}
$$

with $d b_{t} / d\left(1+\lambda_{D E_{t}}\right)<0$. Bonus dynamics then are computed equivalently to wage and effort dynamics.

Concerning total compensation, I focus on the case $\eta^{2} \theta^{3}>1$, hence $w_{t}=\frac{\left(\eta^{2} \theta^{3}\left(1+\lambda_{D E_{t}}\right)-1\right)^{2}}{4 \eta^{3} \theta^{3}\left(1+\lambda_{D E_{t}}\right)^{2}}>$ 0. Then,

$$
\frac{d\left(w_{t}+b_{t}\right)}{d\left(1+\lambda_{D E_{t}}\right)}=\frac{-2 \eta^{2} \theta^{3}\left(1+\lambda_{D E_{t}}\right)+1+\eta^{2} \theta^{3}\left(1+\lambda_{D E_{t}}\right)^{2}-\left(1+\lambda_{D E_{t}}\right)}{4 \eta^{3} \theta^{3}\left(1+\lambda_{D E_{t}}\right)^{4}}
$$

which is negative for $\lambda_{D E_{t}} \rightarrow 0$. To show that this expression can also be positive, note that a binding (DE) constraint delivers

$$
\frac{3 \eta^{2} \theta^{3}\left(1+\lambda_{D E_{t}}\right)-1}{6 \eta^{3} \theta^{3}\left(1+\lambda_{D E_{t}}\right)^{3}}=\delta \Pi_{t+1}
$$

hence $3 \eta^{2} \theta^{3}\left(1+\lambda_{D E_{t}}\right)-1 \geq 0$. At $3 \eta^{2} \theta^{3}\left(1+\lambda_{D E_{t}}\right)-1=0$, the numerator of $d\left(w_{t}+b_{t}\right) / d\left(1+\lambda_{D E_{t}}\right)$ becomes $\left(3 \eta^{2} \theta^{3}-2\right) / 9 \eta^{2} \theta^{3}>0$. 
Proof of Lemma 6. First, I consider the case $w_{t}>0$, hence $\eta^{2} \theta^{3}\left(1+\lambda_{D E_{t}}\right)-1>0$.

Then,

$$
\begin{aligned}
u_{t} & =w_{t}+b_{t}-\frac{e_{t}^{3}}{3}+\eta w_{t} e_{t}^{*} \theta \\
& =w_{t}+2 / 3\left(\sqrt{\eta w_{t} \theta}\right)^{3} \\
& =\frac{\left(\eta^{2} \theta^{3}\left(1+\lambda_{D E_{t}}\right)-1\right)^{2}}{4 \eta^{3} \theta^{3}\left(1+\lambda_{D E_{t}}\right)^{2}}\left[1+\frac{\left(\eta^{2} \theta^{3}\left(1+\lambda_{D E_{t}}\right)-1\right)}{3\left(1+\lambda_{D E_{t}}\right)}\right] \text { and } \\
\frac{\partial u_{t}}{\partial\left(1+\lambda_{D E_{t}}\right)} & =\frac{\left(\eta^{2} \theta^{3}\left(1+\lambda_{D E_{t}}\right)-1\right)}{2 \eta^{3} \theta^{3}\left(1+\lambda_{D E_{t}}\right)^{3}}\left[1+\frac{2\left(\eta^{2} \theta^{3}\left(1+\lambda_{D E_{t}}\right)-1\right)}{6\left(1+\lambda_{D E_{t}}\right)}\right] \\
& +\frac{\left(\eta^{2} \theta^{3}\left(1+\lambda_{D E_{t}}\right)-1\right)^{2}}{12 \eta^{3} \theta^{3}\left(1+\lambda_{D E_{t}}\right)^{3}}>0
\end{aligned}
$$

Moreover,

$$
\begin{aligned}
\pi_{t} & =e \theta-w-b \text { and } \\
\frac{\partial \pi_{t}}{\partial\left(1+\lambda_{D E_{t}}\right)} & =\frac{\partial e}{\partial\left(1+\lambda_{D E_{t}}\right)} \theta-\frac{\partial w}{\partial\left(1+\lambda_{D E_{t}}\right)}-\frac{\partial b}{\partial\left(1+\lambda_{D E_{t}}\right)} \\
& =-\frac{\eta^{2} \theta^{3}\left(1+\lambda_{D E_{t}}\right) \lambda_{D E_{t}}+\left(\eta^{2} \theta^{3}\left(1+\lambda_{D E_{t}}\right)-1\right)}{2 \eta^{3} \theta^{3}\left(1+\lambda_{D E_{t}}\right)^{4}}<0
\end{aligned}
$$

Finally,

$$
\begin{aligned}
\lim _{\lambda_{D E_{t}} \rightarrow \infty} u_{t} & =\frac{\left(\eta^{4} \theta^{6}-\frac{2 \eta^{2} \theta^{3}}{\left(1+\lambda_{D E_{t}}\right)}+\frac{1}{\left(1+\lambda_{D E_{t}}\right)^{2}}\right)}{4 \eta^{3} \theta^{3}}\left[1+\frac{\left(\eta^{2} \theta^{3}-\frac{1}{\left(1+\lambda_{D E_{t}}\right)}\right)}{3}\right] \\
& =\frac{\eta \theta^{3}}{4}\left[1+\frac{\eta^{2} \theta^{3}}{3}\right]=u_{T}
\end{aligned}
$$

and

$$
\begin{aligned}
\lim _{\lambda_{D E_{t} \rightarrow \infty} \pi_{t}} & =\frac{\frac{1}{\left(1+\lambda_{D E_{t}}\right)}+\eta^{2} \theta^{3}}{2 \eta \theta} \theta-\frac{\left(\eta^{4} \theta^{6}-\frac{2 \eta^{2} \theta^{3}}{\left(1+\lambda_{D E_{t}}\right)}+\frac{1}{\left(1+\lambda_{D E_{t}}\right)^{2}}\right)}{4 \eta^{3} \theta^{3}} \\
& -\frac{3 \eta^{2} \theta^{3}-\frac{1}{\left(1+\lambda_{D E_{t}}\right)}}{6 \eta^{3} \theta^{3}\left(1+\lambda_{D E_{t}}\right)^{2}}=\frac{\eta \theta^{3}}{4}=\pi_{T}
\end{aligned}
$$


Second, I consider the case $w_{t}=0$.

Then

$$
\begin{aligned}
u_{t} & =0 \\
\pi_{t} & =\sqrt{\frac{\theta}{\left(1+\lambda_{D E_{t}}\right)}\left(\theta-\frac{\theta}{3\left(1+\lambda_{D E_{t}}\right)}\right) \text { and }} \\
\frac{\partial \pi_{t}}{\partial\left(1+\lambda_{D E_{t}}\right)} & =-\sqrt{\frac{\theta^{3}}{\left(1+\lambda_{D E_{t}}\right)^{3}}} \frac{\lambda_{D E_{t}}}{2\left(1+\lambda_{D E_{t}}\right)}<0
\end{aligned}
$$

Finally, note that $w_{t}>0$ for $\lambda_{D E_{t}}$ sufficiently large, hence the first case also applies to $\lambda_{D E_{t}} \rightarrow \infty$.

Proof of Proposition 2. First, note that $e_{T}^{*}=\eta \theta^{2} / 2$, which is obviously increasing in $\eta$. Second, assume that a positive wage is optimal in any period $t<T$ (i.e., if $\eta>\bar{\eta}$ with a non-binding (DE) constraint and $\eta>\tilde{\eta}$ with a binding (DE) constraint). Then, $e_{t}^{*}=\frac{1+\eta^{2} \theta^{3}\left(1+\lambda_{D E_{t}}\right)}{2 \eta \theta\left(1+\lambda_{D E_{t}}\right)}$, with $\lambda_{D E_{t}} \geq 0$, and

$$
\frac{\partial e_{t}^{*}}{\partial \eta}=\frac{\eta^{2} \theta^{3}\left(1+\lambda_{D E t}\right)-1}{2 \eta^{2} \theta\left(1+\lambda_{D E t}\right)}-\frac{1}{2 \eta \theta\left(1+\lambda_{D E_{t}}\right)^{2}} \frac{\partial \lambda_{D E_{t}}}{\partial \eta}>0
$$

There, $\partial \lambda_{D E_{t}} / \partial \eta \leq 0$ because $\lambda_{D E_{t}}$ is decreasing in $\Pi_{t+1}$ (see the proof to Proposition 1 ), and because profits in all periods increase in $\eta$ : This is obviously true for $\Pi_{T}=$ $\eta \theta^{3} / 4$. Therefore, (DE) constraints in all periods $\tau<T$ are relaxed. Moreover, the agent's (IC) constraints in all periods $\tau<T$ are relaxed by a higher $\eta$ if $w_{\tau}>0$ and stay unaffected if $w_{\tau}=0$.

Now, assume that $w_{t}=0$ is optimal in any period $t<T$. Then, $e_{t}^{*}=\sqrt{\theta /\left(1+\lambda_{D E_{t}}\right)}$, with $\lambda_{D E_{t}} \geq 0$, and

$$
\frac{\partial e_{t}^{*}}{\partial \eta}=-\frac{1}{2} \sqrt{\frac{\theta}{\left(1+\lambda_{D E_{t}}\right)^{3}}} \frac{\partial \lambda_{D E_{t}}}{\partial \eta} \geq 0
$$

The second part $\left(\partial e_{t}^{*} / \partial \eta\right.$ is larger if $\left.\lambda_{D E_{t}}>0\right)$ immediately follows.

Proof of Lemma 7. For a given $w \geq \bar{w}$, the agent chooses an effort level that maximizes $u=w+\eta(w-\bar{w}) \theta e-e^{3} / 3$, hence $e^{*}=\sqrt{\eta(w-\bar{w}) \theta}$. Taking this into account, the principal maximizes profits $\pi=e^{*} \theta-w=\sqrt{\eta(w-\bar{w}) \theta} \theta-w$, subject to $w \geq \bar{w}$. 
First ignoring the latter constraint, the principal's first-order condition equals

$$
\frac{\eta \theta^{2}}{2 \sqrt{\eta(w-\bar{w}) \theta}}-1=0 .
$$

This yields

$$
w=\frac{\eta \theta^{3}}{4}+\bar{w}
$$

which is larger than $\bar{w}$.

Hence,

$$
e^{*}=\frac{\eta \theta^{2}}{2}
$$

and $\pi=\frac{\eta \theta^{3}}{4}-\bar{w}, u=\frac{\eta \theta^{3}}{4}+\frac{\eta^{3} \theta^{6}}{12}+\bar{w}$.

Proof of Proposition 3 Take a profit-maximizing equilibrium with wage sequence $\left\{w_{i}\right\}_{i=1}^{T}$. First note that, in any period $t$, one of the three following cases must hold:

1. (DE) does not bind (i.e., satisfied for first-best wage and effort levels) in period $t$ with $\hat{w}_{\tau \mid t}=0 \forall \tau>t$

2. (DE) does not bind period $t$ with $\hat{w}_{\tau \mid t} \leq w_{\tau} \forall \tau>t$ and at least one $\hat{w}_{\tau \mid t}>0$

3. (DE) binds in period $t$; then $\hat{w}_{\tau \mid t}=w_{\tau} \forall \tau>t$

It cannot be optimal that (DE) binds with $\hat{w}_{\tau \mid t} \leq w_{\tau} \forall \tau>t$ and at least one strict inequality. If this was optimal and there was a $\tau^{\prime}$ with $\hat{w}_{\tau^{\prime} \mid t}<w_{\tau^{\prime}}$, the principal could slightly increase $\hat{w}_{\tau^{\prime} \mid t}$ (such that $\hat{w}_{\tau^{\prime} \mid t}<w_{\tau^{\prime}}$ still holds). This would relax the (DE) constraint at no cost and thus increase profits.

If case 1 holds in a period $t$, it also holds in all previous periods. To show this, I can apply the arguments derived in Lemma 5 and Proposition 1: If (DE) is slack in period $t$, then $\Pi_{t}>\Pi_{t+1}$. Thus, if it is slack in period $t$ with $\hat{w}_{\tau \mid t}=0 \forall \tau>t$, it is also slack in period $t-1$ with $\hat{w}_{\tau \mid t-1}=0 \forall \tau>t-1$.

Moreover, assume case 2 holds in a period $t$ (which implies that there are $\hat{w}_{\tau \mid t} \leq$ $w_{\tau} \forall \tau>t$ and at least one $\hat{w}_{\tau \mid t}>0$ such that (DE) holds as an equality for first-best wage and effort levels). By the arguments derived in Lemma 5 and Proposition 1, this implies $\Pi_{t}>\Pi_{t+1}$ and $\Pi_{t-1}>\Pi_{t}$. Thus, either case 1 or case 2 holds in period $t-1$, and either case 2 or case 3 holds in period $t+1$. If case 2 holds in period $t+1$, $\hat{w}_{\tau \mid t+1} \geq \hat{w}_{\tau \mid t} \forall \tau>t+1$ by construction. Moreover, because $\Pi_{t}>\Pi_{t+1}$, there must be at least one $\tau^{\prime}>t+1$ with $\hat{w}_{\tau^{\prime} \mid t+1}>\hat{w}_{\tau^{\prime} \mid t}$. The latter is also true if case 3 holds in period $t+1$. 
Finally, if case 3 holds in a period $t$, it also holds in all subsequent periods: By construction, if $\hat{w}_{\tau \mid t}=w_{\tau} \forall \tau>t$, then also $\hat{w}_{\tau \mid t+i}=w_{\tau} \forall \tau>t$ and $i \geq 1$. It follows that (DE) must also bind in subsequent periods (again applying the arguments used in Lemma 5 and Proposition 1).

Now, I show that all cases can occur. Given the arguments just derived, it is sufficient to demonstrate that all cases can occur in period $T-1$. In the proof to Lemma 5, I have already shown that without commitment (which is equivalent to having $\hat{w}_{\tau \mid t}=0 \forall \tau>$ t) (DE) might or might not bind. It only remains to show that the same holds with commitment. Thus, assume $\eta>\bar{\eta}$ as defined in the proof to Lemma 3 . Then, firstbest effort and wage levels are $e=\left(1+\eta^{2} \theta^{3}\right) /(2 \eta \theta)$ and $w=\left(\eta^{2} \theta^{3}-1\right)^{2}\left(4 \eta^{3} \theta^{3}\right)$, and the (DE) constraint in period $T-1$ (if $w_{T}$ is the sequentially optimal wage level) becomes

$$
\frac{3 \eta^{2} \theta^{3}-1}{6 \eta^{3} \theta^{3}} \leq \delta\left(\frac{\eta \theta^{3}}{4}+\hat{w}_{T}\right) .
$$

The right hand side increases in $\delta$ and in $\hat{w}_{T}$, and the optimal spot wage equals $w_{T}^{*}=\eta \theta^{3} / 4$. If this condition is satisfied for $\hat{w}_{T} \leq w_{T}^{*}$, cases 1 or 2 apply, otherwise case 3 . The constraint is violated for $\delta \rightarrow 0$, hence case 3 is feasible. Now, fix $\delta>0$; the derivative of the left-hand-side of (DE) equals $\left(1-\eta^{2} \theta^{3}\right) / 2 \eta^{4} \theta^{3}$, which is negative for $\eta>\bar{\eta}$. Moreover, $\lim _{\eta \rightarrow \infty} \frac{3 \eta^{2} \theta^{3}-1}{6 \eta^{3} \theta^{3}}=0$, whereas the right-hand side of (DE) is strictly positive and increasing in $\eta$. Thus, there are values of $\eta$ and $\delta$ such that the condition holds for $\hat{w}_{T} \in\left[0, w_{T}^{*}\right]$.

Concluding, all three cases are feasible in every period. If each of them occurs at some point, it starts with case 1 , followed by case 2 and then case 3 . If only cases 1 and 2 occur, then it starts with case 1 , and so on.

Now, I explore wage dynamics. In the proof to Proposition 1, I have already shown that wages and effort are constant if (DE) does not bind, which here applies to cases 1 and 2.

For the following, assume that (DE) binds in at least one period and take the earliest period $t(\geq 1)$ in which it does. From this period on, $\hat{w}_{\tau \mid t}=w_{\tau} \forall \tau>t$ and the optimization problem is not sequentially efficient anymore. In period $t$, the principal determines $w_{t}$ as well as all future wages $\left\{w_{i}\right\}_{i=t}^{T}$. Thus, the Lagrange function of the underlying optimization problem equals 


$$
\begin{aligned}
L_{t}= & e_{t} \theta-e_{t}^{3} / 3+\eta w_{t} e_{t} \theta-2 / 3\left(\sqrt{\eta w_{t} \theta}\right)^{3}-w_{t} \\
& +\delta\left[e_{t+1} \theta-e_{t+1}^{3} / 3+\eta w_{t+1} e_{t+1} \theta-2 / 3\left(\sqrt{\eta w_{t+1} \theta}\right)^{3}-w_{t+1}\right]+\ldots \\
& +\lambda_{D E_{t}}\left[\delta\left(\Pi_{t+1}+\hat{w}_{t+1}+\delta \hat{w}_{t+2}+\ldots\right)-\frac{2}{3}\left(\sqrt{\eta w_{t} \theta}\right)^{3}-e_{t}^{3} / 3+\eta w_{t} \theta e_{t}\right] \\
& +\delta \lambda_{D E_{t+1}}\left[\delta\left(\Pi_{t+2}+\hat{w}_{t+2}+\delta \hat{w}_{t+3}+\ldots\right)-\frac{2}{3}\left(\sqrt{\eta w_{t+1} \theta}\right)^{3}-e_{t+1}^{3} / 3+\eta w_{t+1} \theta e_{t+1}\right]+\ldots \\
& +w_{t} \lambda_{w_{t}}+\delta w_{t+1} \lambda_{w_{t+1}} \ldots
\end{aligned}
$$

First-order conditions for $i \geq 0$ are

$$
\begin{aligned}
\frac{\partial L}{\partial w_{t+i}} & =\delta^{i}\left[\eta \theta\left(e_{t+i}-\sqrt{\eta w_{t+i} \theta}\right)\left(1+\sum_{j=0}^{i} \lambda_{D E_{t+j}}\right)-1+\lambda_{w_{t+i}}\right]=0 \\
& \Rightarrow\left(e_{t+i}-\sqrt{\eta w_{t+i} \theta}\right)\left(1+\sum_{j=0}^{i} \lambda_{D E_{t+j}}\right)=\frac{1-\lambda_{w_{t+i}}}{\eta \theta} \\
\frac{\partial L}{\partial e_{t+i}} & =\delta^{i}\left[\theta\left(1+\sum_{j=0}^{i-1} \lambda_{D E_{t+j}}\right)-\left(e_{t+i}^{2}-\eta w_{t+i} \theta\right)\left(1+\sum_{j=0}^{i} \lambda_{D E_{t+j}}\right)\right]=0 \\
& \Rightarrow\left(e_{t+i}-\sqrt{\eta w_{t+i} \theta}\right)\left(1+\sum_{j=0}^{i} \lambda_{D E_{t+j}}\right)=\frac{\theta\left(1+\sum_{j=0}^{i-1} \lambda_{D E_{t+j}}\right)}{\left(e_{t+i}+\sqrt{\eta w_{t+i} \theta}\right)} \\
& \Rightarrow 2 \sqrt{\eta w_{t+i} \theta}=\frac{\eta \theta^{2}\left(1+\sum_{j=0}^{i-1} \lambda_{D E_{t+j}}\right)}{1-\lambda_{w_{t+i}}}-\frac{1-\lambda_{w_{t+i}}}{\eta \theta\left(1+\sum_{j=0}^{i} \lambda_{D E_{t+j}}\right)}
\end{aligned}
$$

Now, I show that if $\lambda_{w_{t+i}}=0$ (and hence $w_{t+i}>0$ ), $w_{t+i+1}>w_{t+i}$. Thus, assume $\lambda_{w_{t+i}}=0$ and set $\lambda_{w_{t+i+1}}=0$ as well (I will show ex post that this holds). Then,

$$
\begin{aligned}
& 2 \sqrt{\eta w_{t+i+1} \theta}-2 \sqrt{\eta w_{t+i} \theta} \\
& =\eta \theta^{2} \sum_{j=0}^{i} \lambda_{D E_{t+j}}+\frac{\lambda_{D E_{t+i+1}}}{\eta \theta\left(1+\sum_{j=0}^{i} \lambda_{D E_{t+j}}\right)\left(1+\sum_{j=0}^{i+1} \lambda_{D E_{t+j}}\right)}>0
\end{aligned}
$$

which confirms that $w_{t+i+1}>w_{t+i}\left(\right.$ and also that $\left.\lambda_{w_{t+i+1}}=0\right)$.

Proof of Proposition 4. In any period $t$, the principal maximizes 


$$
\pi_{t}=e_{t} \theta-\left(\left(e_{t}\right)^{3} / 3-\eta\left(w_{t}-\bar{w}\right) e_{t} \theta+2 / 3\left(\sqrt{\eta\left(w_{t}-\bar{w}\right) \theta}\right)^{3}\right)-w_{t}, \text { subject to (DE) }
$$

and $w_{t} \geq \bar{w}$. First, I assume that (DE) does not bind (which is possible if $\eta$ and/or $\delta$ are sufficiently large - see the proof to Lemma 3 ). Then, the Lagrange function equals

$$
\begin{aligned}
L_{t}= & e_{t} \theta-\left(e_{t}\right)^{3} / 3+\eta\left(w_{t}-\bar{w}\right) e_{t} \theta \\
& -\frac{2}{3}\left(\sqrt{\eta\left(w_{t}-\bar{w}\right) \theta}\right)^{3}-w_{t}+\lambda_{w_{t}}\left(w_{t}-\bar{w}\right),
\end{aligned}
$$

with first order conditions

$$
\begin{aligned}
& \frac{\partial L_{t}}{\partial e_{t}^{*}}=\theta-\left(e_{t}\right)^{2}+\eta\left(w_{t}-\bar{w}\right) \theta=0 \\
& \frac{\partial L_{t}}{\partial w_{t}}=\eta \theta\left(e_{t}-\sqrt{\eta\left(w_{t}-\bar{w}\right) \theta}\right)-1+\lambda_{w_{t}}=0
\end{aligned}
$$

I start with $\lambda_{w_{t}}=0$. Then, the first order conditions yield $w_{t}=\left(\eta^{2} \theta^{3}-1\right)^{2} /\left(4 \eta^{3} \theta^{3}\right)+$ $\bar{w}$ and $e_{t}^{*}=\left(1+\eta^{2} \theta^{3}\right) /(2 \eta \theta)$. Now, assume that $\lambda_{w_{t}}>0$ and hence $w_{t}=\bar{w}$. Then, $e_{t}^{*}=\sqrt{\theta}$. Moreover, note that $d \pi_{t} /\left.d w_{t}\right|_{w_{t}=\bar{w}}=\sqrt{\eta^{2} \theta^{3}}-1$. This is positive for $\eta>$ $\sqrt{1 / \theta^{3}}$, hence a strictly positive wage is optimal in this case and not otherwise. Therefore, effort levels in both cases $\left(w_{t}>0\right.$ and $\left.w_{t}=0\right)$ are not affected by $\bar{w}$, as well as the threshold $\bar{\eta}$ above which $w_{t}>0$ is optimal. Therefore, equilibrium effort is independent of $\bar{w}$. It follows that $e_{t}^{*}$ and $w_{t}$ are independent of $\bar{\Pi}$.

Now, I include the respective (DE) constraints, which yields the Lagrange function of the principal's maximization problem in a period $t$

$$
\begin{aligned}
L_{t} & =e_{t} \theta-e_{t}^{3} / 3+\eta\left(w_{t}-\bar{w}\right) e_{t} \theta-2 / 3\left(\sqrt{\eta\left(w_{t}-\bar{w}\right) \theta}\right)^{3}-w_{t} \\
& +\lambda_{D E_{t}}\left[\delta\left(\Pi_{t+1}-\bar{\Pi}\right)-\frac{2}{3}\left(\sqrt{\eta\left(w_{t}-\bar{w}\right) \theta}\right)^{3}-e_{t}^{3} / 3+\eta\left(w_{t}-\bar{w}\right) \theta e_{t}\right] \\
& +\lambda_{w_{t}}\left(w_{t}-\bar{w}\right),
\end{aligned}
$$

where $\lambda_{w_{t}} \geq 0$ represents the Lagrange parameter for the agent's limited liability constraint and $\lambda_{D E_{t}} \geq 0$ represents the Lagrange parameter for the principal's dynamic enforcement constraint. 
First-order conditions are

$$
\begin{aligned}
\frac{\partial L}{\partial e_{t}^{*}}= & \theta-e_{t}^{2}+\eta\left(w_{t}-\bar{w}\right) \theta+\lambda_{D E_{t}}\left[-e_{t}^{2}+\eta\left(w_{t}-\bar{w}\right) \theta\right]=0 \\
\frac{\partial L}{\partial w_{t}}= & \eta \theta e_{t}-\eta \theta \sqrt{\eta\left(w_{t}-\bar{w}\right) \theta}-1 \\
& +\lambda_{D E_{t}}\left[-\eta \theta \sqrt{\eta\left(w_{t}-\bar{w}\right) \theta}+\eta \theta e_{t}\right]+\lambda_{w_{t}}=0 .
\end{aligned}
$$

I start with $\lambda_{w_{t}}=0$. Then, the first order conditions yield $w_{t}=\frac{\left(\left(1+\lambda_{D E_{t}}\right) \eta^{2} \theta^{3}-1\right)^{2}}{\left(1+\lambda_{D E_{t}}\right)^{2} 4 \eta^{3} \theta^{3}}+\bar{w}$ and $e_{t}=\frac{1+\left(1+\lambda_{D E_{t}}\right) \eta^{2} \theta^{3}}{\left(1+\lambda_{D E_{t}}\right) 2 \eta \theta}$. It follows that, given $\lambda_{D E_{t}}>0$ and $\eta^{2} \theta^{3}-1 \geq 0, \frac{\left(\eta^{2} \theta^{3}\left(1+\lambda_{D E_{t}}\right)-1\right)^{2}}{4 \eta^{3} \theta^{3}\left(1+\lambda_{D E_{t}}\right)^{2}}>\frac{\left(\eta^{2} \theta^{3}-1\right)^{2}}{4 \eta^{3} \theta^{3}}$ and $\frac{1+\eta^{2} \theta^{3}\left(1+\lambda_{D E_{t}}\right)}{2 \eta \theta\left(1+\lambda_{D E_{t}}\right)}<\frac{1+\eta^{2} \theta^{3}}{2 \eta \theta}$.

Now, assume that $\lambda_{w_{t}}>0$ and hence $w_{t}=\bar{w}$. Then, $e_{t}^{*}=\sqrt{\theta /\left(1+\lambda_{D E_{t}}\right)}$. To show that both cases, $w_{t}=\bar{w}$ and $w_{t}>\bar{w}$, are feasible, note that $\partial L /\left.\partial w_{t}\right|_{w_{t}=\bar{w}}=$ $\sqrt{\eta^{2} \theta^{3}\left(1+\lambda_{D E_{t}}\right)}-1=0$. This is positive for $\eta>\sqrt{1 / \theta^{3}\left(1+\lambda_{D E_{t}}\right)}$, hence a strictly positive wage is optimal in this case and not otherwise.

Now, I show that $\Pi_{t}$ is decreasing in $\bar{w}$. This implies that (DE) is more likely to bind for a higher $\bar{w}$, and thus - once (DE) binds $-\lambda_{D E_{t}}$ increases with $\bar{w}$ (see the proof to Lemma 5). First, I have already shown (in the proof to Lemma 7) that $\Pi_{T}=\pi_{T}$ is decreasing in $\bar{w}$. Therefore, (DE) in period $T-1$ is tightened, and consequently profits $\pi_{T-1}$ and $\Pi_{T-1}$ are reduced for larger values of $\bar{w}$. This tightens the (DE) constraint in period $T-2$ and reduces profits $\pi_{T-2}$ and $\Pi_{T-2}$, and so on. Therefore, $\Pi_{t}$ is decreasing in $\bar{w}$ for all $t$ and $\lambda_{D E_{t}}$, if positive, is increasing.

Therefore,

$$
\begin{aligned}
\frac{\partial e_{t}^{*}}{\partial \bar{w}} & =-\frac{1}{2 \eta \theta\left(1+\lambda_{D E_{t}}\right)^{2}} \frac{\partial \lambda_{D E_{t}}}{\partial \bar{w}}<0 \\
\frac{\partial w_{t}}{\partial \bar{w}} & =\frac{\left(1+\lambda_{D E_{t}}\right) \eta^{2} \theta^{3}-1}{2 \eta^{3} \theta^{3}\left(1+\lambda_{D E_{t}}\right)^{3}} \frac{\partial \lambda_{D E_{t}}}{\partial \bar{w}}+1>1 .
\end{aligned}
$$

if $w_{t}>\bar{w}$. If $w_{t}=\bar{w}$

$$
\begin{aligned}
& \frac{\partial e_{t}^{*}}{\partial \bar{w}}=-\frac{1}{2} \sqrt{\frac{\theta}{\left(1+\lambda_{D E_{t}}\right)^{3}}} \frac{\partial \lambda_{D E_{t}}}{\partial \bar{w}}<0 \\
& \frac{\partial w_{t}}{\partial \bar{w}}=1 .
\end{aligned}
$$

Finally, $\lambda_{D E_{t}}$ increases in $\bar{\Pi}$ because a larger $\bar{\Pi}$ tigthens (DE) (see the proof to Lemma $5)$. 
Therefore,

$$
\begin{aligned}
& \frac{\partial e_{t}^{*}}{\partial \bar{\Pi}}=-\frac{1}{2 \eta \theta\left(1+\lambda_{D E_{t}}\right)^{2}} \frac{\partial \lambda_{D E_{t}}}{\partial \bar{\Pi}}<0 \\
& \frac{\partial w_{t}}{\partial \bar{\Pi}}=\frac{\left(\left(1+\lambda_{D E_{t}}\right) \eta^{2} \theta^{3}-1\right)}{2 \eta^{3} \theta^{3}\left(1+\lambda_{D E_{t}}\right)^{3}} \frac{\partial \lambda_{D E_{t}}}{\partial \bar{\Pi}}+1>1 .
\end{aligned}
$$

Proof of Proposition 5. First, I show that, for $p \rightarrow 1$, a separating contract yields higher profits than a pooling contract. There, note that, in any profit-maximizing equilibrium, (ICS), the selfish type's (IC) constraint, is tighter than (ICR), the reciprocal type's (IC) constraint:

$$
\begin{aligned}
& -\frac{e_{1}^{3}}{3}+\delta w_{2} \geq 0 \\
& -\frac{e_{1}^{3}}{3}+\eta w_{1} \theta e_{1}+\delta\left[w_{2}+\frac{2\left(\sqrt{\eta w_{2} \theta}\right)^{3}}{3}\right] \\
& \geq \frac{2}{3}\left(\sqrt{\eta w_{1} \theta}\right)^{3} .
\end{aligned}
$$

With $w_{1}=0$, (ICS) is tighter than (ICR) for any second-period wage $w_{2}$ because second-period utilities are larger for the reciprocal type. A strictly positive $w_{1}$ can only possibly be optimal for the principal if it further relaxes (ICR) ((ICS) is unaffected by $w_{1}$ ), which confirms that (ICS) is tighter than (ICR) in any profit-maximizing equilibrium. This implies that a strictly higher effort level can be implemented with a separating contract (then however only exerted by the reciprocal type) than with a pooling contract (then exerted by both). For $p \rightarrow 1$, profits under both regimes approach $e_{1} \theta-w_{1}+\delta\left(\sqrt{w_{2} \eta \theta} \theta-w_{2}\right)$, which is larger with a separating contract because of the higher effort implemented in this case.

To show that a pooling contract yields higher profits than a separating contract for $p \rightarrow 0$, I first assume that the principal offers a pooling contract and explore its properties. Then, I do the same with a separating contract, and finally compare both alternatives.

Pooling contract In any profit-maximizing equilibrium, (ICS) is tighter than (ICR). Therefore, (ICS) determines feasible effort in a pooling contract. This also implies that $w_{1}=0$, because a positive $w_{1}$ might only relax (ICR).

Now, the principal maximizes $\Pi_{1}$, subject to her own (DE) constraint, $p e_{2} \theta-w_{2} \geq 0$, 
as well as the selfish agent's (IC) constraint, $-\frac{e_{1}^{3}}{3}+\delta w_{2} \geq 0$. This will bind because, otherwise, the principal could ask for a higher first-period effort level without violating any constraint. Moreover, the reciprocal type exerts an effort level $e_{2}=\sqrt{w_{2} \eta \theta}$ in the second period, whereas the selfish type's second period effort amounts to zero, hence $\Pi_{1}=e_{1} \theta+\delta\left(p \sqrt{w_{2} \eta \theta} \theta-w_{2}\right)$.

Taking all this into account, the Lagrange function becomes

$$
L=e_{1} \theta+\delta\left[p \sqrt{\frac{e_{1}^{3}}{3 \delta} \eta \theta \theta}-\frac{e_{1}^{3}}{3 \delta}\right]+\lambda_{D E}\left[p \sqrt{\frac{e_{1}^{3}}{3 \delta} \eta \theta \theta}-\frac{e_{1}^{3}}{3 \delta}\right],
$$

and the first order condition

$$
\frac{\partial L}{\partial e_{1}}=\theta+\left[\frac{p \eta \theta^{2}}{2 \sqrt{\frac{e_{1}^{3}}{3 \delta} \eta \theta}}-1\right] \frac{e_{1}^{2}}{\delta}\left(\delta+\lambda_{D E}\right)=0 .
$$

First, assume $\lambda_{D E}=0$. Then, $e_{1}^{*}$ is characterized by

$$
2 \sqrt{\frac{\eta \theta}{3 \delta}}\left(\theta-\left(e_{1}^{*}\right)^{2}\right)+p \eta \theta^{2} \sqrt{e_{1}^{*}}=0
$$

Second, assume $\lambda_{D E}>0$. Then, $e_{1}^{*}$ is determined by the binding (DE) constraint,

$$
e_{1}^{*}=\sqrt[3]{3 \delta p^{2} \eta \theta^{3}}
$$

To compute the condition for when (DE) actually binds, I plug $e_{1}^{*}=\sqrt[3]{3 \delta p^{2} \eta \theta^{3}}$ into the first order condition,

$$
\begin{gathered}
\theta+\left[\frac{p \eta \theta^{2}}{2 \sqrt{\frac{e_{1}^{3}}{3 \delta} \eta \theta}}-1\right] \frac{e_{1}^{2}}{\delta}\left(\delta+\lambda_{D E}\right) \\
=\theta-\frac{1}{2} \frac{e_{1}^{2}}{\delta}\left(\delta+\lambda_{D E}\right) \\
=\theta-\frac{1}{2} \frac{\left(\sqrt[3]{3 \delta p^{2} \eta \theta^{3}}\right)^{2}}{\delta}\left(\delta+\lambda_{D E}\right)=0 .
\end{gathered}
$$


Therefore, (DE) binds if $\theta-\frac{1}{2} \theta^{2}\left(\sqrt[3]{3 \delta p^{2} \eta}\right)^{2} \geq 0$, or

$$
p^{2} \leq \frac{\left(\sqrt{\frac{2}{\theta}}\right)^{3}}{3 \delta \eta}
$$

In this case, which is the relevant case for $p \rightarrow 0$, the principal's profits with a pooling equilibrium are

$$
\Pi_{1}^{P}=e_{1}^{*} \theta=\sqrt[3]{3 \delta p^{2} \eta} \theta^{2}
$$

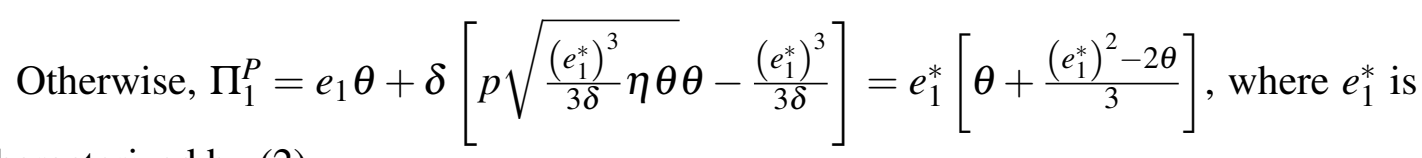
characterized by (2).

Separating contract In case she offers a separating contract, the principal maximizes $\Pi_{1}=p\left[e_{1} \theta+\delta\left(e_{2} \theta-w_{2}\right)\right]-w_{1}$, where $e_{2}=\sqrt{w_{2} \eta \theta}$, subject to her own (DE) constraint, $e_{2} \theta-w_{2} \geq 0$ (which is relevant in case the agent turns out to be reciprocal), the limited liability constraint, $w_{1} \geq 0$, as well as the reciprocal agent's binding (IC) constraint,

$$
\begin{aligned}
& -\frac{\left(e_{1}^{*}\right)^{3}}{3}+\eta w_{1} \theta e_{1}^{*}+\delta\left[w_{2}+\frac{2\left(\sqrt{\eta w_{2} \theta}\right)^{3}}{3}\right] \\
= & \frac{2\left(\sqrt{\eta w_{1} \theta}\right)^{3}}{3} .
\end{aligned}
$$

There, note that

$$
\begin{aligned}
\frac{d e_{1}^{*}}{d w_{1}} & =\eta \theta \frac{e_{1}^{*}-\sqrt{\eta w_{1} \theta}}{\left(e_{1}^{*}\right)^{2}-\eta w_{1} \theta}=\frac{\eta \theta}{e_{1}^{*}+\sqrt{\eta w_{1} \theta}} \\
\frac{d e_{1}^{*}}{d w_{2}} & =\frac{\delta\left[1+\sqrt{\eta w_{2} \theta} \eta \theta\right]}{\left(e_{1}^{*}\right)^{2}-\eta w_{1} \theta}
\end{aligned}
$$

Therefore, the Lagrange function becomes $L=p\left[e_{1} \theta+\delta\left(\sqrt{w_{2} \eta \theta} \theta-w_{2}\right)+\lambda_{D E}\left(\sqrt{w_{2} \eta \theta} \theta-w_{2}\right)\right]-$ 
$w_{1}+\lambda_{w_{1}} w_{1}$, with first order conditions

$$
\begin{aligned}
\frac{\partial L}{\partial w_{1}} & =p \frac{\eta \theta}{e_{1}+\sqrt{\eta w_{1} \theta}} \theta-1+\lambda_{w_{1}}=0 \\
\frac{\partial L}{\partial w_{2}} & =p\left[\frac{\delta\left[1+\sqrt{\eta w_{2} \theta} \eta \theta\right]}{e_{1}^{2}-\eta w_{1} \theta} \theta+\delta\left(\frac{\eta \theta^{2}}{2 \sqrt{w_{2} \eta \theta}}-1\right)+\lambda_{D E}\left(\frac{\eta \theta^{2}}{2 \sqrt{w_{2} \eta \theta}}-1\right)\right]=0
\end{aligned}
$$

For later use, note that the first condition implies that $w_{1}=0$ for $p \rightarrow 0$ (because $e_{1}^{*}$ is bounded away from zero for any strictly positive $\delta$ ).

First, assume $\lambda_{D E}=0$, hence

$$
\frac{1+\sqrt{\eta w_{2} \theta} \eta \theta}{e_{1}^{2}-\eta w_{1} \theta} \theta+\frac{\eta \theta^{2}}{2 \sqrt{w_{2} \eta \theta}}-1=0
$$

This, together with the reciprocal agent's (IC) constraint, determines outcomes if $w_{1}=0$. If $w_{1}>0$, outcomes are additionally given by

$$
p \frac{\eta \theta^{2}}{e_{1}^{*}+\sqrt{\eta w_{1} \theta}}-1=0
$$

and an explicit characterization of the results is not feasible.

Now, assume $\lambda_{D E}>0$. Then, a binding (DE) constraint implies $w_{2}=\eta \theta^{3}$.

If $w_{1}=0$, (IC) yields

$$
e_{1}^{*}=\sqrt[3]{3 \delta\left[\eta \theta^{3}+\frac{2 \eta^{3} \theta^{6}}{3}\right]}
$$

To compute the condition for when $w_{1}=0$ (if (DE) binds), I plug these values into the first first order condition, $p \frac{\eta \theta^{2}}{\sqrt[3]{3 \delta\left[\eta \theta^{3}+\frac{2 \eta^{3} \theta^{6}}{3}\right]}}-1+\lambda_{w_{1}}=0$. Therefore, $w_{1}=0$ if

$$
\begin{gathered}
p \frac{\eta \theta^{2}}{\sqrt[3]{3 \delta\left[\eta \theta^{3}+\frac{2 \eta^{3} \theta^{6}}{3}\right]}}-1 \leq 0 \\
p^{3} \leq \frac{3 \delta\left[\eta \theta^{3}+\frac{2 \eta^{3} \theta^{6}}{3}\right]}{\eta^{3} \theta^{6}}
\end{gathered}
$$

To compute the condition for when (DE) binds (if $w_{1}=0$ ), I plug these values into 
the second first order condition. Therefore, (DE) binds for

$$
\frac{\delta\left[1+\eta \theta^{2} \eta \theta\right]}{\left(\sqrt[3]{3 \delta\left[\eta \theta^{3}+\frac{2 \eta^{3} \theta^{6}}{3}\right]}\right)^{2}} \theta-\frac{1}{2} \delta \geq 0
$$

or

$$
\delta^{2} \leq \frac{8 \theta^{3}\left[1+\eta^{2} \theta^{3}\right]^{3}}{9\left[\eta \theta^{3}+\frac{2 \eta^{3} \theta^{6}}{3}\right]^{2}} .
$$

The right hand side of this condition is larger than 1, and (DE) always binds if $w_{1}=0$. Therefore, (DE) always binds if $p \rightarrow 0$ because then, $w_{1}=0$ (see above). On a general note, though, wIwant to emphasize that this might change in a more general setup with a longer time horizon.

All this implies that, for $p \rightarrow 0$, profits with a separating contract are

$$
\Pi_{1}^{S}=p e_{1}^{*} \theta=p \theta \sqrt[3]{3 \delta\left[\eta \theta^{3}+\frac{2 \eta^{3} \theta^{6}}{3}\right]}
$$

Comparison For $p \rightarrow 0$, profits with a pooling contract are $\Pi_{1}^{P}=\sqrt[3]{3 \delta p^{2} \eta} \theta^{2}$, and $\Pi_{1}^{S}=p \theta \sqrt[3]{3 \delta\left[\eta \theta^{3}+\frac{2 \eta^{3} \theta^{6}}{3}\right]}$ for a separating contract. Therefore,

$$
\begin{aligned}
& \Pi_{1}^{P}>\Pi_{1}^{S} \\
\Leftrightarrow & \sqrt[3]{3 \delta p^{2} \eta \theta^{3}} \theta \geq p \theta \sqrt[3]{3 \delta\left[\eta \theta^{3}+\frac{2 \eta^{3} \theta^{6}}{3}\right]} \\
\Leftrightarrow & 1 \geq p\left(1+\frac{2 \eta^{2} \theta^{3}}{3}\right)
\end{aligned}
$$

which holds for $p \rightarrow 0$.

Proof of Lemma 8. First, I give a more precise description of optimal outcomes:

If $\eta^{2} \theta^{3}>\frac{3+\sqrt{9-6 \delta}}{3 \delta}$, (DE) does not bind. Then, $\hat{w}_{2}=0$ and $w_{1}>0$.

For $\eta^{2} \theta^{3} \leq \frac{3+\sqrt{9-6 \delta}}{3 \delta}$, (DE) binds. In this case,

- $w_{1}>0$ and $\hat{w}_{2}=0$ if

$$
\delta \leq \eta^{2} \theta^{3} \frac{3-\sqrt{3}}{2}
$$

- $w_{1}>0$ and $\hat{w}_{2}>0$ if

$$
\delta \in \eta^{2} \theta^{3}\left(\frac{3-\sqrt{3}}{2}, 1\right)
$$


Moreover, $w_{2}>\hat{w}_{2}$.

- $w_{1}=0$ and $\hat{w}_{2}>0$ if $\delta>\eta^{2} \theta^{3}$ (and $\eta^{2} \theta^{3}<1$ ). Moreover, $w_{2}>\hat{w}_{2}$ if $\delta<$ $2.25 \eta^{2} \theta^{3}$, and $w_{2}=\hat{w}_{2}$ and $\delta \geq 2.25 \eta^{2} \theta^{3}$.

Proof: The principal sets $e_{1}, b_{1}, w_{1}$ and $\hat{w}_{2}$ to maximize

$$
\Pi_{1}=e_{1} \theta-w_{1}-b_{1}+\delta\left(\sqrt{w_{2} \eta \theta} \theta-w_{2}\right)-(1-\delta) \hat{w}_{2}
$$

subject to

$$
\begin{aligned}
b_{1}-\frac{\left(e_{1}^{*}\right)^{3}}{3}+\eta w_{1} e_{1}^{*} \theta & \geq 2 / 3\left(\sqrt{\eta w_{1} \theta}\right)^{3} \\
-b_{1}+\delta\left(\sqrt{w_{2} \eta \theta} \theta-w_{2}+\hat{w}_{2}\right) & \geq 0 \\
w_{1} & \geq 0 \\
\hat{w}_{2} & \geq 0
\end{aligned}
$$

In the second period (if $\theta$ has not dropped to zero), the principal sets $w_{2}$ to maximize $\pi_{2}$, subject to the constraint $w_{2} \geq \hat{w}_{2}$. This is also taken into account by the principal when determining her period-1 actions. First, though, we omit this constraint and check ex-post whether $\hat{w}_{2}$ is indeed below the optimal second-period spot contract, $\eta \theta^{3} / 4$. In this case, $\pi_{2}=\eta \theta^{3} / 4$.

As before, (IC) binds in a profit-maximizing equilibrium. Solving (IC) for $b_{1}$ and substituting it into $\Pi_{1}$ and (DE) yields the Lagrange function

$$
\begin{aligned}
L= & e_{1} \theta-\frac{e_{1}^{3}}{3}+\eta w_{1} e_{1} \theta-2 / 3\left(\sqrt{\eta w_{1} \theta}\right)^{3}-w_{1}+\delta \pi_{2}-(1-\delta) \hat{w}_{2} \\
& +\lambda_{D E}\left[-\frac{e_{1}^{3}}{3}+\eta w_{1} e_{1} \theta-\frac{2}{3}\left(\sqrt{\eta w_{1} \theta}\right)^{3}+\delta\left(\pi_{2}+\hat{w}_{2}\right)\right] \\
& +\lambda_{\hat{w}_{2}} \hat{w}_{2}+\lambda_{w_{1}} w_{1},
\end{aligned}
$$


with first-order conditions

$$
\begin{aligned}
\frac{\partial L}{\partial e_{1}} & =\theta+\left(\eta w_{1} \theta-e_{1}^{2}\right)\left(1+\lambda_{D E}\right)=0 \\
& \Rightarrow\left(1+\lambda_{D E}\right)=\frac{\theta}{\left(e_{1}^{2}-\eta w_{1} \theta\right)} \\
\frac{\partial L}{\partial w_{1}} & =\eta \theta\left(e_{1}-\sqrt{\eta w_{1} \theta}\right)\left(1+\lambda_{D E}\right)-1+\lambda_{w_{1}}=0 \\
& \Rightarrow \lambda_{w_{1}}=1-\frac{\eta \theta^{2}}{\left(e_{1}+\sqrt{\eta w_{1} \theta}\right)} \\
\frac{\partial L}{\partial \hat{w}_{2}} & =-(1-\delta)+\delta \lambda_{D E}+\lambda_{\hat{w}_{2}}=0 \\
& \Rightarrow \lambda_{\hat{w}_{2}}=1-\delta\left(1+\lambda_{D E}\right)=1-\delta \frac{\theta}{\left(e_{1}^{2}-\eta w_{1} \theta\right)}
\end{aligned}
$$

The last condition already reveals that $\hat{w}_{2}=0$ if $\lambda_{D E}=0$.

In the following, I analyze all potential cases and derive conditions for each of those to hold.

\section{1.) Slack (DE)}

This case mirrors the results derived in Lemma 3, derived for the specific case of two periods: $\lambda_{D E}=0$ yields

$$
e_{1}^{2}=\theta\left(1+\eta w_{1}\right)
$$

and we have to disginguish between the two cases $w_{1}=0$ and $w_{1}>0$.

A) $w_{1}=0 \quad$ First-order conditions yield $\lambda_{w_{1}}>0 \Leftrightarrow e_{1}>\eta \theta^{2}$, whereas $e_{1}=\sqrt{\theta}$ follows from (3), hence $\eta^{2} \theta^{3}<1$ is a necessary condition for this case to hold.

Finally, a slack (DE) constraint at these values requires

$$
\eta^{2} \theta^{3} \geq\left(\frac{4}{3 \delta}\right)^{2}
$$

which is at odds with $\eta^{2} \theta^{3}<1$. Hence, if only one period is left, a non-binding (DE) constraint requires $w_{1}>0$ (for $\delta \leq 1$ ), also see the proof to Lemma 5 .

B) $w_{1}>0$ First-order conditions yield $\lambda_{w_{1}}=0 \Leftrightarrow w_{1}=\frac{\left(\eta \theta^{2}-e_{1}\right)^{2}}{\eta \theta}$, whereas $e_{1}=$ $\frac{1+\eta^{2} \theta^{3}}{2 \eta \theta}-$ and consequently $w_{1}=\left(\frac{\eta^{2} \theta^{3}-1}{2 \eta \theta}\right)^{2} / \eta \theta-$ follows from (3). 
Also note that $\lambda_{w_{1}}=1-\frac{\eta \theta^{2}}{\left(e_{1}+\sqrt{w_{1} \eta \theta}\right)}=0 \Leftrightarrow \sqrt{w_{1} \eta \theta}=\eta \theta^{2}-e_{1}$ and $e_{1} \leq \eta \theta^{2}$. This implies $\eta^{2} \theta^{3} \geq 1$, and a non-binding (DE) constraint at these values requires

$$
-\frac{\left(\frac{1+\eta^{2} \theta^{3}}{2 \eta \theta}\right)^{3}}{3}+\left(\frac{\eta^{2} \theta^{3}-1}{2 \eta \theta}\right)^{2} \frac{1+\eta^{2} \theta^{3}}{2 \eta \theta}-2 / 3\left(\sqrt{\left(\frac{\eta^{2} \theta^{3}-1}{2 \eta \theta}\right)^{2}}\right)^{3}+\delta \frac{\eta \theta^{3}}{4} \geq 0
$$

Since $\eta^{2} \theta^{3} \geq 1$, this condition becomes

$$
2-6 \eta^{2} \theta^{3}+3 \delta \eta^{4} \theta^{6} \geq 0
$$

and either holds for $\eta^{2} \theta^{3}>(3+\sqrt{9-6 \delta}) / 3 \delta$ or $\eta^{2} \theta^{3}<(3-\sqrt{9-6 \delta}) / 3 \delta$. Again, since $\eta^{2} \theta^{3} \geq 1$, (DE) does not bind for

$$
\eta^{2} \theta^{3}>\frac{3+\sqrt{9-6 \delta}}{3 \delta}
$$

\section{2.) Binding (DE)}

Assume (DE) binds, hence $\eta^{2} \theta^{3} \leq(3+\sqrt{9-6 \delta}) / 3 \delta$. Moreover, first-order conditions reveal that, since $\lambda_{D E}>0, e_{1}^{2}<\theta\left(1+\eta w_{1}\right)$. In the following, I explore all four potential cases separately and assess the conditions for each of them to hold.

A) $w_{1}=0, \hat{w}_{2}=0 \quad$ First-order conditions yield $\lambda_{w_{1}}>0 \Leftrightarrow 1-\frac{\eta \theta^{2}}{\left(e_{1}+\sqrt{\eta w_{1} \theta}\right)}>0 \Leftrightarrow e_{1}>$ $\eta \theta^{2}$. Recall that $e_{1}^{2}<\theta$ must hold as well, hence $w_{1}=0$ if the necessary condition

$$
\eta^{2} \theta^{3}<1
$$

is satisfied.

Moreover, a binding (DE) constraint, taking into account $w_{1}=\hat{w}_{2}=0$, yields $e_{1}=$ $\sqrt[3]{3 \delta \pi_{2}}$

$\lambda_{\hat{w}_{2}}>0$ also requires $1-\delta \frac{\theta}{\left(e_{1}^{2}-\eta w_{1} \theta\right)}>0$, which is equivalent to

$$
\delta<\frac{\left(3 \pi_{2}\right)^{2}}{\theta^{3}}
$$

However, recall that $\lambda_{w_{1}}>0$ requires $e_{1}>\eta \theta^{2}$, hence $\delta>\eta^{3} \theta^{6} /\left(3 \pi_{2}\right)$. A necessary condition for both constraints on $\delta$ to hold is $\eta \theta^{3}<3 \pi_{2}$ which, for spot profits $\pi_{2}=$ 
$\eta \theta^{3} / 4$, becomes

$$
1<\frac{3}{4}
$$

a contradiction. Thus, this case can be ruled out.

B) $w_{1}=0, \hat{w}_{2}>0 \quad$ As in case A), first-order conditions yield $\lambda_{w_{1}}>0 \Leftrightarrow 1-\frac{\eta \theta^{2}}{\left(e_{1}+\sqrt{w_{1} \eta \theta}\right)}>$ $0 \Leftrightarrow e_{1}>\eta \theta^{2}$, as well as $e_{1}^{2}<\theta$, which give the necessary condition

$$
\eta^{2} \theta^{3}<1
$$

Moreover, $\lambda_{\hat{w}_{2}}=1-\delta \frac{\theta}{e_{1}^{2}}=0 \Leftrightarrow e_{1}=\sqrt{\delta \theta}$, and a binding (DE) constraint yields

$$
\hat{w}_{2}=\frac{\sqrt{\delta \theta^{3}}}{3}-\pi_{2}
$$

Thus, $\hat{w}_{2}$ is below the unconstrained spot wage, $\eta \theta^{3} / 4$ (in which case $\pi_{2}=\eta \theta^{3} / 4$ ), if

$$
\delta \leq \frac{9}{4} \eta^{2} \theta^{3}
$$

If this conditions is satisfied,

$$
\hat{w}_{2}=\frac{\sqrt{\delta \theta^{3}}}{3}-\frac{\eta \theta^{3}}{4}>0 \Leftrightarrow \delta>\left(\frac{3}{4}\right)^{2} \eta^{2} \theta^{3},
$$

which holds given $\eta^{2} \theta^{3}<1$.

Now, let us assume $\delta>2.25 \eta^{2} \theta^{3}$, hence $\hat{w}_{2}$ would be above optimal the optimal spot contract. In this case, $w_{2}=\hat{w}_{2}$ which the principal takes into account when setting $\hat{w}_{2}$. Note that here we can stick to having $w_{1}=0 ; w_{1}>0$ is considered in the next section.

Hence, for $\delta>2.25 \eta^{2} \theta^{3}$, the Lagrange function becomes

$$
\begin{aligned}
L= & e_{1} \theta-\frac{e_{1}^{3}}{3}+\delta\left(\sqrt{w_{2} \eta \theta} \theta-w_{2}\right)-(1-\delta) w_{2} \\
& +\lambda_{D E}\left[-\frac{e_{1}^{3}}{3}+\delta\left(\sqrt{w_{2} \eta \theta} \theta\right)\right]
\end{aligned}
$$

with 


$$
\begin{aligned}
\frac{\partial L}{\partial e_{1}} & =\theta-e_{1}^{2}\left(1+\lambda_{D E}\right)=0 \\
& \Rightarrow\left(1+\lambda_{D E}\right)=\frac{\theta}{e_{1}^{2}} \\
\frac{\partial L}{\partial w_{2}} & =\frac{\delta \eta \theta^{2}}{2 \sqrt{w_{2} \eta \theta}}\left(1+\lambda_{D E}\right)-1=0 \\
& \Rightarrow \sqrt{w_{2} \eta \theta}=\frac{\delta \eta \theta^{3}}{2 e_{1}^{2}} .
\end{aligned}
$$

Plugging this into the binding (DE) constraint yields

$$
e_{1}=\left(\frac{3 \delta^{2} \eta \theta^{4}}{2}\right)^{\frac{1}{5}}
$$

and

$$
w_{2}=\left(\frac{\delta^{2} \eta \theta^{9}}{2^{6} 3^{4}}\right)^{\frac{1}{5}}
$$

Regarding consistency, note that $\left(\frac{\delta^{2} \eta \theta^{9}}{2^{6} 3^{4}}\right)^{\frac{1}{5}}>\frac{\eta \theta^{3}}{4} \Leftrightarrow \delta>2.25 \eta^{2} \theta^{3}$.

C) $w_{1}>0, \hat{w}_{2}>0 \quad$ Now, first-order conditions yield $\lambda_{w_{1}}=0 \Leftrightarrow 1-\frac{\eta \theta^{2}}{\left(e_{1}+\sqrt{\eta w_{1} \theta}\right)}=$ $0 \Leftrightarrow \sqrt{\eta w_{1} \theta}=\eta \theta^{2}-e_{1} \Rightarrow \eta \theta^{2} \geq e_{1}$

Moreover, $\lambda_{w_{1}}=0 \Leftrightarrow w_{w_{1}}=\frac{\left(\eta \theta^{2}-e_{1}\right)^{2}}{\eta \theta}$, as well as $\lambda_{\hat{w}_{2}}=0 \Leftrightarrow 1-\delta \frac{\theta}{\left(e_{1}^{2}-\eta w_{1} \theta\right)}=0$.

Combining these two conditions delivers

$$
e_{1}=\frac{\delta+\eta^{2} \theta^{3}}{2 \eta \theta}
$$

This is indeed smaller than $\eta \theta^{2}$ for

$$
\delta \leq \eta^{2} \theta^{3}
$$

Finally, a binding (DE) constraint (with $\pi_{2}=\eta \theta^{3} / 4$ ) yields

$$
\hat{w}_{2}=\frac{3 \delta \eta^{2} \theta^{3}-\delta^{2}}{6 \eta^{3} \theta^{3}}-\frac{\eta \theta^{3}}{4} .
$$

Before going on, I check whether this value is below the unconstrained spot wage, $\eta \theta^{3} / 4: \hat{w}_{2} \leq \eta \theta^{3} / 4 \Leftrightarrow \delta^{2}+\eta^{2} \theta^{3}\left(3 \eta^{2} \theta^{3}-\delta\right) \geq 0$, which always holds for $\delta \leq \eta^{2} \theta^{3}$.

Hence, it only remains to derive the consistency conditions for $\hat{w}_{2}$ indeed being opti- 
mal. Solving for the respective values of $\delta$ reveals that $\hat{w}_{2}$ is positive for

$$
\delta \in\left(\eta^{2} \theta^{3} \frac{3-\sqrt{3}}{2}, \eta^{2} \theta^{3} \frac{3+\sqrt{3}}{2}\right) .
$$

Since $\frac{3+\sqrt{3}}{2}>1$ and $\delta \leq \eta^{2} \theta^{3}$ because of $e_{1} \leq \eta \theta^{2}$, this case holds for

$$
\delta \in \eta^{2} \theta^{3}\left(\frac{3-\sqrt{3}}{2}, 1\right)
$$

D) $w_{1}>0, \hat{w}_{2}=0 \quad$ It follows that this case holds for $\delta \leq \eta^{2} \theta^{3} \frac{3-\sqrt{3}}{2}$

Proof of Lemma 10. The principal maximizes

$$
\Pi_{1}=e_{1} \theta-\left(e_{1}^{*}\right)^{3} / 3+\eta w_{1} e_{1}^{*} \theta-2 / 3\left(\sqrt{\eta w_{1} \theta}\right)^{3}-w_{1}+\delta\left(\frac{\eta \theta^{3}}{4}-w_{1}\right),
$$

subject to $w_{1} \geq 0$ and

$$
\frac{\left(e_{1}^{*}\right)^{3}}{3}-\eta w_{1} \theta e_{1}^{*}+\frac{2}{3}\left(\sqrt{\eta w_{1} \theta}\right)^{3} \leq \delta\left(\frac{\eta \theta^{3}}{4}-w_{1}\right)
$$

This yields the Lagrange function

$$
\begin{aligned}
L & =e_{1} \theta-\left(e_{1}\right)^{3} / 3+\eta w_{1} e_{1} \theta-2 / 3\left(\sqrt{\eta w_{1} \theta}\right)^{3}-w_{1}+\delta\left(\frac{\eta \theta^{3}}{4}-w_{1}\right) \\
& +\lambda_{w_{1}} w_{1}+\lambda_{D E}\left[\eta w_{1} \theta e_{1}+\delta\left(\frac{\eta \theta^{3}}{4}-w_{1}\right)-\frac{2}{3}\left(\sqrt{\eta w_{1} \theta}\right)^{3}-\frac{\left(e_{1}\right)^{3}}{3}\right]
\end{aligned}
$$

where $\lambda_{w_{1}} \geq 0$ represents the Lagrange parameter for the agent's limited liability constraint, and $\lambda_{D E} \geq 0$ the Lagrange parameter for the principal's dynamic enforcement constraint.

First order conditions are

$$
\begin{aligned}
\frac{\partial L}{\partial e_{1}}= & \theta-e_{1}^{2}+\eta w_{1} \theta+\lambda_{D E}\left[\eta w_{1} \theta-e_{1}^{2}\right]=0 \\
\frac{\partial L}{\partial w_{1}}= & \eta e_{1} \theta-\eta \theta \sqrt{\eta w_{1} \theta}-1-\delta+\lambda_{w_{1}} \\
& +\lambda_{D E}\left[\eta \theta e_{1}^{*}-\delta-\eta \theta \sqrt{\eta w_{1} \theta}\right]=0 .
\end{aligned}
$$


First, assume $\lambda_{D E}=0$. Then, I have to consider the two cases $w_{1}=0$ and $w_{1}>0$.

If $w_{1}=0, e_{1}^{*}=\sqrt{\theta}$ and $\Pi_{1}=2 / 3(\sqrt{\theta})^{3}+\delta \eta \theta^{3} / 4$. Moreover, $d \Pi_{1} /\left.d w_{1}\right|_{w_{1}=0}=$ $\sqrt{\eta^{2} \theta^{3}}-1-\delta$, therefore $w_{1}=0$ for $\eta^{2} \theta^{3} \leq(1+\delta)^{2}$, whereas $w_{1}>0$ for $\eta^{2} \theta^{3}>$ $(1+\delta)^{2}$. Recall that the condition for a positive wage in case (DE) is not binding in the main part (i.e., without an adjustment of the reference wage) equals $\eta^{2} \theta^{3}>1$.

Furthermore, $e_{1}^{*}>e_{2}^{*} \Leftrightarrow \eta^{2} \theta^{3}<4$, which holds because $\eta^{2} \theta^{3}<(1+\delta)^{2}$. Moreover, $0=w_{1}<w_{2}=\frac{\eta \theta^{3}}{4}$, and $\frac{d e_{1}^{*}}{d \eta}=0<\frac{d e_{2}^{*}}{d \eta}$.

To check the feasibility of the case $\lambda_{D E}=0$ and $w_{1}=0$, I plug the respective values into the (DE) constraint, and obtain

$$
\frac{16}{9 \delta^{2}} \leq \eta^{2} \theta^{3}
$$

This is consistent with $\eta^{2} \theta^{3} \leq(1+\delta)^{2}$ if $3 \delta(1+\delta) \geq 4$. Now, assume $\eta^{2} \theta^{3}>$ $(1+\delta)^{2}$ and $\lambda_{D E}=0$. Hence, $\lambda_{w_{1}}=0$, and the first order conditions yield $e_{1}=$ $\frac{(1+\delta)^{2}+\eta^{2} \theta^{3}}{2 \eta \theta(1+\delta)}$ and $w_{1}=\frac{\left[\eta^{2} \theta^{3}-(1+\delta)^{2}\right]^{2}}{4(1+\delta)^{2} \eta^{3} \theta^{3}}$. Moreover, $e_{1}^{*}>e_{2}^{*} \Leftrightarrow \delta \theta^{3} \eta^{2}<(1+\delta)^{2}$, which only is consistent with $\eta^{2} \theta^{3}>(1+\delta)^{2}$ if $\delta$ is sufficiently small. In any case, $w_{1}<w_{2}$ and $d e_{1}^{*} / d \eta<d e_{2}^{*} / d \eta$, where the latter condition is equivalent to $\delta \eta^{2} \theta^{3}>-(1+\delta)^{2}$.

To check the feasibility of the case $\lambda_{D E}=0$ and $w_{1}>0$, I plug the respective values into the (DE) constraint, and obtain

$$
2 \leq \delta\left(\frac{(1+\delta)^{2} \eta^{2} \theta^{3}-1}{(1+\delta)^{2}}\right)+(1+\delta)^{2} \frac{1}{3} \frac{(2-\delta)}{\eta^{2} \theta^{3}} .
$$

The right hand side is increasing in $\eta^{2}$ if $\delta$ is large enough. Since $\eta^{2} \theta^{3}>(1+\delta)^{2}$, this condition holds if it is satisfied for $\eta^{2} \theta^{3}=(1+\delta)^{2}$. For this case, it becomes

$$
\frac{4}{3} \leq \delta^{2}(2+\delta)+\frac{2}{3} \delta-\frac{\delta}{(1+\delta)^{2}}
$$

There, the right hand side is increasing in $\delta$ and, for $\delta \rightarrow 1$, approaches $3+\frac{5}{12}>\frac{4}{3}$. Hence, this case is feasible if $\eta$ and/or $\delta$ are large enough.

Now, assume that the (DE) constraint binds, hence $\lambda_{D E}>0$.

First, I assume that $\lambda_{w_{t}}>0$, hence $w_{t}=0$ and $e_{t}^{*}=\sqrt{\theta /\left(1+\lambda_{D E_{t}}\right)}$. To establish the existence of $\tilde{\eta}$, note that $\partial L /\left.\partial w_{t}\right|_{w_{t}=0}=\left(\eta \theta \sqrt{\theta /\left(1+\lambda_{D E_{t}}\right)}-\delta\right)\left(1+\lambda_{D E}\right)-1$, which is positive for $\eta^{2} \theta^{3}>\left(1+\delta\left(1+\lambda_{D E}\right)\right)^{2} /\left(1+\lambda_{D E}\right)$. This threshold is larger than with a non-binding (DE) if $\lambda_{D E}>\left(1-\delta^{2}\right) / \delta^{2}$, which might or might not hold. Moreover, provided $\eta^{2} \theta^{3} \leq\left(1+\delta\left(1+\lambda_{D E}\right)\right)^{2} /\left(1+\lambda_{D E}\right), e_{1}^{*}>e_{2}^{*} \Leftrightarrow \eta^{2} \theta^{3}\left(1+\lambda_{D E_{t}}\right)<$ 4 , which might or might not hold. 
Second, I assume $\eta^{2} \theta^{3}>\left(1+\delta\left(1+\lambda_{D E}\right)\right)^{2} /\left(1+\lambda_{D E}\right)$, hence $\lambda_{w_{1}}=0$. Then, the first order conditions yield

$$
\begin{gathered}
e_{1}=\frac{\eta^{2} \theta^{3}\left(1+\lambda_{D E}\right)+\left(1+\delta\left(1+\lambda_{D E}\right)\right)^{2}}{2 \eta \theta\left(1+\lambda_{D E}\right)\left(1+\delta\left(1+\lambda_{D E}\right)\right)} \\
w_{1}=\frac{\left[\eta^{2} \theta^{3}\left(1+\lambda_{D E}\right)-\left(1+\delta\left(1+\lambda_{D E}\right)\right)^{2}\right]^{2}}{4 \eta^{3} \theta^{3}\left(1+\lambda_{D E}\right)^{2}\left(1+\delta\left(1+\lambda_{D E}\right)\right)^{2}} .
\end{gathered}
$$

Now, $e_{1}^{*}>e_{2}^{*} \Leftrightarrow \frac{\left(1+\delta\left(1+\lambda_{D E}\right)\right)^{2}}{\delta\left(1+\lambda_{D E}\right)^{2}}>\eta^{2} \theta^{3}$, which might or might not be consistent with $\eta^{2} \theta^{3}>\left(1+\delta\left(1+\lambda_{D E}\right)\right)^{2} /\left(1+\lambda_{D E}\right)$.

Proof of Lemma 11. In the second period, the principal maximizes $\pi_{2}=e_{2} \theta-w_{2}$, where $e_{2}$ is given by

$$
-e_{2}^{2}-\frac{4}{3} e_{2}^{3} \eta \theta+w_{2} \eta \theta=0
$$

This yields

$$
\begin{aligned}
& e_{2}^{*}=\frac{\sqrt{1+4 \eta^{2} \theta^{3}}-1}{4 \eta \theta} \\
& w_{2}=\frac{e_{2}^{2}+\frac{4}{3} e_{2}^{3} \eta \theta}{\eta \theta} \\
& \pi_{2}=e_{2}^{*}\left(\frac{8 \eta^{2} \theta^{3}+1-\sqrt{1+4 \eta^{2} \theta^{3}}}{12 \eta^{2} \theta^{2}}\right) \\
& u_{2}=\frac{\left(e_{2}^{*}\right)^{2}\left(1+\eta e_{2}^{*} \theta\right)^{2}}{\eta \theta} .
\end{aligned}
$$

Recall that last-period profits in the main setup are $\eta \theta^{3} / 4$, which is larger than the amount obtained here.

In the first period, at $e_{1}^{*}, u_{1}$ is decreasing in $e_{1}$. If it were increasing, the agent would further raise his effort level. This implies that (IC) is binding in a profit-maximizing equilibrium. If it were not binding, the principal could ask for a higher effort level without paying more.

Plugging the binding (IC) constraint,

$$
b_{1}=\frac{\left(e_{t}^{*}\right)^{3}}{3}-w_{1}+\left(w_{1}-\frac{\left(\tilde{e}_{t}\right)^{3}}{3}\right) \frac{\left(1+\eta \tilde{e}_{1} \theta\right)}{\left(1+\eta e_{1}^{*} \theta\right)},
$$

into profits and the (DE) constraint yields the Lagrange function 


$$
\begin{aligned}
L & =e_{1} \theta-\frac{\left(e_{t}^{*}\right)^{3}}{3}-\left(w_{1}-\frac{\left(\tilde{e}_{t}\right)^{3}}{3}\right) \frac{\left(1+\eta \tilde{e}_{1} \theta\right)}{\left(1+\eta e_{1}^{*} \theta\right)} \\
& +\lambda_{w_{1}} w_{1}+\lambda_{D E}\left[-\frac{\left(e_{1}^{*}\right)^{3}}{3}+w_{1}-\left(w_{1}-\frac{\left(\tilde{e}_{1}\right)^{3}}{3}\right) \frac{\left(1+\eta \tilde{e}_{1} \theta\right)}{\left(1+\eta e_{1}^{*} \theta\right)}+\delta \pi_{2}\right]
\end{aligned}
$$

where $\lambda_{w_{1}} \geq 0$ represents the Lagrange parameter for the agent's limited liability constraint, and $\lambda_{D E} \geq 0$ the Lagrange parameter for the principal's dynamic enforcement constraint.

First order conditions are

$$
\begin{aligned}
\frac{\partial L}{\partial e_{1}}= & \theta-\left(e_{1}^{*}\right)^{2}+\left(w_{1}-\frac{\left(\tilde{e}_{t}\right)^{3}}{3}\right) \frac{\eta \theta\left(1+\eta \tilde{e}_{1} \theta\right)}{\left(1+\eta e_{1}^{*} \theta\right)^{2}} \\
& +\lambda_{D E}\left[-\left(e_{t}^{*}\right)^{2}+\left(w_{1}-\frac{\left(\tilde{e}_{t}\right)^{3}}{3}\right) \frac{\eta \theta\left(1+\eta \tilde{e}_{1} \theta\right)}{\left(1+\eta e_{1}^{*} \theta\right)^{2}}\right]=0 \\
\frac{\partial L}{\partial w_{1}}= & -\left(1-(\tilde{e})^{2} \frac{d \tilde{e}}{d w_{1}}\right) \frac{(1+\eta \tilde{e} \theta)}{\left(1+\eta e_{1}^{*} \theta\right)}-\left(w_{1}-\frac{(\tilde{e})^{3}}{3}\right) \frac{\eta \theta}{\left(1+\eta e_{1}^{*} \theta\right)} \frac{d \tilde{e}}{d w_{1}} \\
& +\lambda_{D E}\left[1-\left(1-(\tilde{e})^{2} \frac{d \tilde{e}}{d w_{1}}\right) \frac{(1+\eta \tilde{e} \theta)}{\left(1+\eta e_{1}^{*} \theta\right)}-\left(w_{1}-\frac{\left(\tilde{e}_{t}\right)^{3}}{3}\right) \frac{\eta \theta}{\left(1+\eta e_{1}^{*} \theta\right)} \frac{d \tilde{e}}{d w_{1}}\right] \\
& +\lambda_{w_{1}}=0
\end{aligned}
$$

Using $-\tilde{e}_{1}^{2}-4 / 3 \tilde{e}_{1}^{3} \eta \theta+w_{1} \eta \theta=0$, which implies $w_{1}=\tilde{e}_{1}^{2} / \eta \theta+4 / 3 \tilde{e}_{1}^{3}$, those conditions become

$$
\begin{aligned}
& \frac{\partial L}{\partial e_{1}}: \theta-\left(\left(e_{1}^{*}\right)^{2}-\tilde{e}_{1}^{2} \frac{\left(1+\eta \tilde{e}_{1} \theta\right)^{2}}{\left(1+\eta e_{1}^{*} \theta\right)^{2}}\right)\left(1+\lambda_{D E}\right)=0 \\
& \frac{\partial L}{\partial w_{1}}:-\frac{(1+\eta \tilde{e} \theta)}{\left(1+\eta e_{1}^{*} \theta\right)}\left(1+\lambda_{D E}\right)+\lambda_{w_{1}}+\lambda_{D E}=0
\end{aligned}
$$

First, assume $\lambda_{D E}=0$. Then, I have to consider the two cases $w_{1}=0$ and $w_{1}>0$. However, $w_{1}>0$ and consequently $\lambda_{w_{1}}=0$ cannot be optimal, since in this case, the second condition would become $-(1+\eta \tilde{e} \theta) /\left(1+\eta e_{1}^{*} \theta\right)=0$.

Therefore, $\lambda_{D E}=0$ implies $w_{1}=0$; hence $\tilde{e}=0$ and

$$
e_{1}^{*}=\sqrt{\theta}
$$

Moreover $e_{1}^{*}=\sqrt{\theta}>\left(\sqrt{1+4 \eta^{2} \theta^{3}}-1\right) /(4 \eta \theta)=e_{2}^{*}$ and $w_{1}=0<\left(e_{2}^{2}+\frac{4}{3} e_{2}^{3} \eta \theta\right) /(\eta \theta)=$ 
$w_{2}$.

However, note that for two periods and $\delta \leq 1, \lambda_{D E}=0$ is not feasible: For $w_{1}=0$, $b_{1}=(\sqrt{\theta})^{3} / 3$ and $e_{1}^{*}=\sqrt{\theta}$, the (DE) constraint becomes

$$
-\frac{(\sqrt{\theta})^{3}}{3}+\delta \frac{\left(1+4 \eta^{2} \theta^{3}\right) \sqrt{1+4 \eta^{2} \theta^{3}}-6 \eta^{2} \theta^{3}-1}{24 \eta^{3} \theta^{3}} \geq 0 .
$$

There, the second term increases in $\eta$ and approaches $\delta 2 \sqrt{\theta^{3}} / 9$ for $\eta \rightarrow \infty$. Therefore, the constraint does not hold for any $\theta$ and $\eta$ if $\delta \leq 1$.

Now, assume that (DE) binds. Again, I start with $w_{1}=0$. Then, $e_{1}^{*}=\sqrt{\theta /\left(1+\lambda_{D E}\right)}$, and

$$
\begin{aligned}
\lim _{w_{1} \rightarrow 0} \frac{\partial L}{\partial w_{1}} & =-\frac{\left(1+\lambda_{D E}\right)}{\left(1+\eta e_{1}^{*} \theta\right)}+\lambda_{D E} \\
& =-\frac{\left(1+\lambda_{D E}\right)}{\left(1+\sqrt{\frac{\eta^{2} \theta^{3}}{\left(1+\lambda_{D E}\right)}}\right)}+\lambda_{D E}
\end{aligned}
$$

which is positive for

$$
\eta^{2} \theta^{3}>\frac{1+\lambda_{D E}}{\lambda_{D E}^{2}}
$$

Put differently,

$$
e_{1}^{*}=\sqrt{\frac{\theta}{\left(1+\lambda_{D E}\right)}}
$$

if $\eta^{2} \theta^{3} \lambda_{D E}^{2}-\lambda_{D E}-1 \leq 0$, hence if $\lambda_{D E} \leq\left(1+\sqrt{1+4 \eta^{2} \theta^{3}}\right) /\left(2 \eta^{2} \theta^{3}\right)$. In this case,

$$
\begin{aligned}
e_{1}^{*} \geq \sqrt{\frac{\theta}{\left(1+\frac{1+\sqrt{1+4 \eta^{2} \theta^{3}}}{2 \eta^{2} \theta^{3}}\right)}} \\
=\sqrt{\frac{2 \eta^{2} \theta^{4}}{2 \eta^{2} \theta^{3}+1+\sqrt{1+4 \eta^{2} \theta^{3}}}}
\end{aligned}
$$

This is larger than $e_{2}^{*}=\left(\sqrt{1+4 \eta^{2} \theta^{3}}-1\right) /(4 \eta \theta)$, if

$$
12 \eta^{4} \theta^{6}>0
$$

Therefore, $e_{1}^{*}>e_{2}^{*}$ and $w_{1}<w_{2}$.

Now, assume that $\lambda_{D E}>\left(1+\sqrt{1+4 \eta^{2} \theta^{3}}\right) /\left(2 \eta^{2} \theta^{3}\right)$, hence $w_{1}>0$. Solving the 
first first order condition for $\lambda_{D E}$ and plugging it into the second yields

$$
\eta \theta^{2}-\frac{\left[1+\eta \theta\left(e_{1}^{*}+\tilde{e}_{1}\right)\right]\left[e_{1}^{*}\left(1+e_{1}^{*} \eta \theta\right)+\tilde{e}_{1}\left(1+\eta \tilde{e}_{1} \theta\right)\right]}{\left(1+\eta e_{1}^{*} \theta\right)}=0
$$

which, together with the binding (DE) constraint, determines $e_{1}^{*}$ as well as $\tilde{e}_{1}$ (and consequently $w_{1}$ ). Making use of $-\tilde{e}_{1}^{2}-\frac{4}{3} \tilde{e}_{1}^{3} \eta \theta+w_{1} \eta \theta=0 \Rightarrow w_{1}=\frac{\tilde{e}_{1}^{2}}{\eta \theta}+\frac{4}{3} \tilde{e}_{1}^{3}$, the latter becomes

$$
-\frac{\left(\left(e_{1}^{*}\right)^{3}-\tilde{e}_{1}^{3}\right)}{3}+\tilde{e}_{1}^{2}\left(e_{1}^{*}-\tilde{e}_{1}\right) \frac{\left(1+\tilde{e}_{1} \eta \theta\right)}{\left(1+\eta e_{1}^{*} \theta\right)}+\delta \pi_{2}=0
$$

In order to prove $e_{1}^{*}>e_{2}^{*}$ and $w_{1}<w_{2}$, I first show that $e_{1}^{*}$ is increasing and $\tilde{e}_{1}$ is decreasing in $\delta \pi_{2}$ :

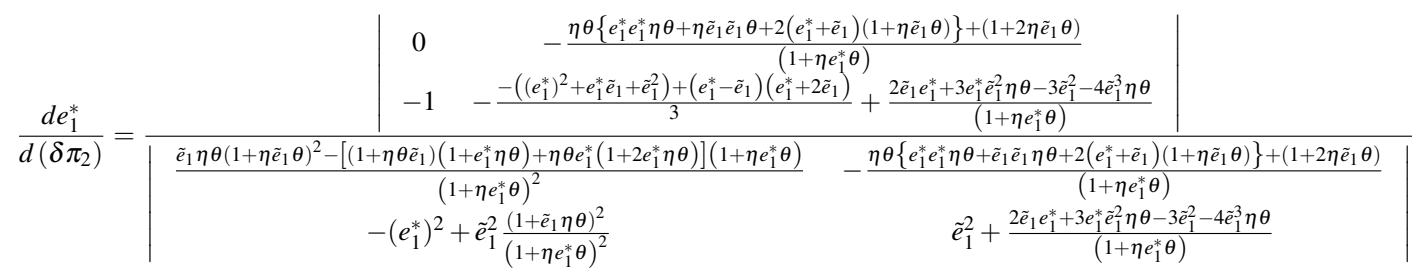

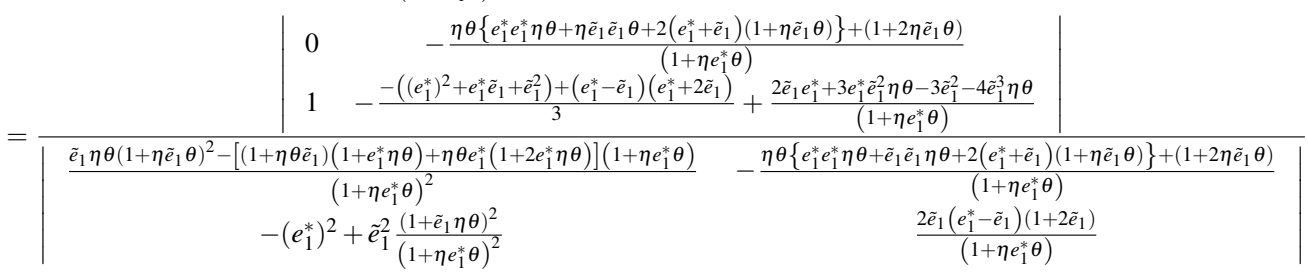

There, the numerator equals

$$
-\frac{\eta \theta\left\{e_{1}^{*} e_{1}^{*} \eta \theta+\eta \tilde{e}_{1} \tilde{e}_{1} \theta+2\left(e_{1}^{*}+\tilde{e}_{1}\right)\left(1+\eta \tilde{e}_{1} \theta\right)\right\}+\left(1+2 \eta \tilde{e}_{1} \theta\right)}{\left(1+\eta e_{1}^{*} \theta\right)}<0,
$$

and the denominator

$$
\begin{aligned}
& \frac{\tilde{e}_{1} \eta \theta\left(1+\eta \tilde{e}_{1} \theta\right)^{2}-\left(\eta \theta \tilde{e}_{1}+1\right)\left(1+e_{1}^{*} \eta \theta\right)^{2}-\eta \theta e_{1}^{*}\left(1+2 e_{1}^{*} \eta \theta\right)\left(1+\eta e_{1}^{*} \theta\right)}{\left(1+\eta e_{1}^{*} \theta\right)^{2}}\left[2\left(e_{1}^{*}-\tilde{e}_{1}\right) \tilde{e}_{1} \frac{1+2 \tilde{e}_{1} \eta \theta}{\left(1+\eta e_{1}^{*} \theta\right)}\right] \\
& +\left(-\left(e_{1}^{*}\right)^{2}+\tilde{e}_{1}^{2} \frac{\left(1+\tilde{e}_{1} \eta \theta\right)^{2}}{\left(1+\eta e_{1}^{*} \theta\right)^{2}}\right) \frac{\eta \theta\left\{e_{1}^{*} e_{1}^{*} \eta \theta+\tilde{e}_{1} \tilde{e}_{1} \eta \theta+2\left(e_{1}^{*}+\tilde{e}_{1}\right)\left(1+\eta \tilde{e}_{1} \theta\right)\right\}+\left(1+2 \eta \tilde{e}_{1} \theta\right)}{\left(1+\eta e_{1}^{*} \theta\right)},
\end{aligned}
$$

which is negative because of $e_{1}^{*}>\tilde{e}_{1}$. Therefore,

$$
\frac{d e_{1}^{*}}{d\left(\delta \pi_{2}\right)}>0
$$

If $\delta \pi_{1}=0, b_{1}=0$, and $\pi_{1}$ is maximized by setting $w_{1}=w_{2}$, implying $e_{1}^{*}=e_{2}^{*}$. 
Therefore, $e_{1}^{*}>e_{2}^{*}$ given $\delta \pi_{1}>0$.

Moreover,

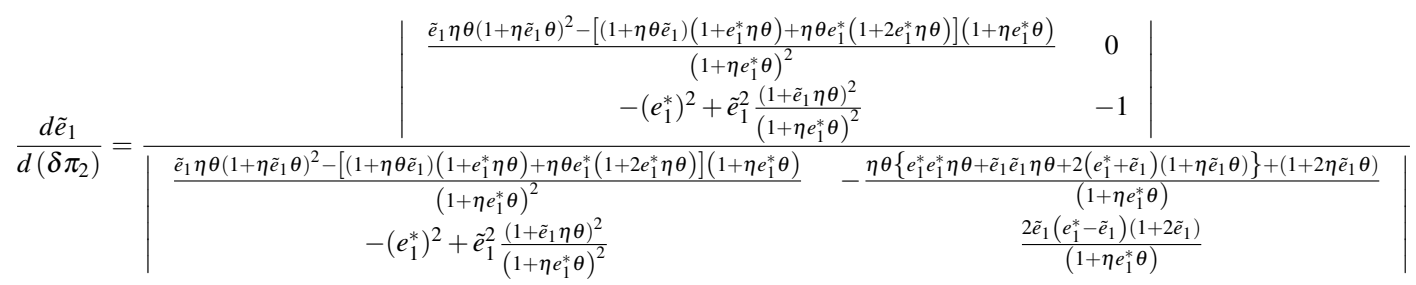

This is negative, since the denominator is negative and the numerator, which equals

$$
\frac{\left[\left(1+\eta \theta \tilde{e}_{1}\right)\left(1+e_{1}^{*} \eta \theta\right)+\eta \theta e_{1}^{*}\left(1+2 e_{1}^{*} \eta \theta\right)\right]\left(1+\eta e_{1}^{*} \theta\right)-\tilde{e}_{1} \eta \theta\left(1+\eta \tilde{e}_{1} \theta\right)^{2}}{\left(1+\eta e_{1}^{*} \theta\right)^{2}}
$$

is positive.

Therefore,

$$
\frac{d w_{1}}{d\left(\delta \pi_{2}\right)}<0
$$

If $\delta \pi_{1}=0, b_{1}=0$, and $\pi_{1}$ is maximized by setting $w_{1}=w_{2}$. Therefore, $w_{1}<w_{2}$ given $\delta \pi_{1}>0$. 


\section{References}

Abreu, D. (1988). On the theory of infinitely repeated games with discounting. Econometrica, 56(2):383-396.

Akerlof, G. A. (1982). Labor contracts as partial gift exchange. Quarterly Journal of Economics, 97(4):543-569.

Akerlof, G. A. and Kranton, R. E. (2000). Economics and identity. Quarterly Journal of Economics, 115(3):715-753.

Akerlof, G. A. and Kranton, R. E. (2005). Identity and the economics of organizations. Journal of Economic Perspectives, 19(1):9-32.

Bellemare, C. and Shearer, B. (2009). Gift giving and worker productivity: Evidence from a firm-level experiment. Games and Economic Behavior, 67:233-244.

Bewley, T. F. (1999). Why Wages Don't Fall During a Recession. Harvard Univ. Press.

Bloom, N. and Reenen, J. V. (2011). Chapter 19 - human resource management and productivity. volume 4 of Handbook of Labor Economics, pages 1697 - 1767. Elsevier.

Boosey, L. and Goerg, S. (2018). The timing of discretionary bonuses: Effort, signals, and reciprocity. IZA Discussion Paper No. 11580.

Brown, M., Falk, A., and Fehr, E. (2004). Relational contracts and the nature of market interactions. Econometrica, 72(3):747-780.

Bull, C. (1987). The existence of self-enforcing implicit contracts. The Quarterly Journal of Economics, 102(1):147-159.

Burdett, K. and Mortensen, D. T. (1998). Wage differentials, employer size, and unemployment. International Economic Review, 39(2):257-273.

Cabral, L., Ozbay, E. Y., and Schotter, A. (2014). Intrinsic and instrumental reciprocity: An experimental study. Games and Economic Behavior, 87:100-121.

Camerer, C. and Weber, R. (2013). Experimental organizational economics. In Gibbons, R. and Roberts, J., editors, The Handbook of Organizational Economics, pages 213 - 262. Princeton University Press.

Cho, I.-K. and Kreps, D. M. (1987). Signaling games and stable equilibria. The Quarterly Journal of Economics, 102(2):179-221. 
Cox, J. C., Friedman, D., and Gjerstad, S. (2007). A tractable model of reciprocity and fairness. Games and Economic Behavior, 59(1):17 - 45.

Cox, J. C., Friedman, D., and Sadiraj, V. (2008). Revealed altruism. Econometrica, 76(1):31-69.

DellaVigna, S. (2009). Psychology and economics: Evidence from the field. Journal of Economic Literature, 47(2):315-372.

DellaVigna, S., Lindner, A., Reizer, B., and Schmieder, J. F. (2017). ReferenceDependent Job Search: Evidence from Hungary. The Quarterly Journal of Economics, 132(4):1969-2018.

DellaVigna, S., List, J. A., Malmendier, U., and Rao, G. (2019). Estimating social preferences and gift exchange with a piece-rate design. Unpublished Working Paper.

DellaVigna, S. and Pope, D. (2018). What Motivates Effort? Evidence and Expert Forecasts. The Review of Economic Studies, 85(2):1029-1069.

Dohmen, T., Falk, A., Huffman, D., and Sunde, U. (2009). Homo reciprocans: Survey evidence on behavioural outcomes. Economic Journal, 119(536):592-612.

Dreber, A., Fudenberg, D., and Rand, D. G. (2014). Who cooperates in repeated games: The role of altruism, inequity aversion, and demographics. Journal of Economic Behavior \& Organization, 98:41-55.

Dufwenberg, M., Heidhues, P., Kirchsteiger, G., Riedel, F., and Sobel, J. (2011). Otherregarding preferences in general equilibrium. The Review of Economic Studies, 78(2):613-639.

Dufwenberg, M. and Kirchsteiger, G. (2004). A theory of sequential reciprocity. Games and Economic Behavior, 47(2):268 - 298.

Dur, R. and Tichem, J. (2015). Altruism and relational incentives in the workplace. Journal of Economics \& Management Strategy, 24(3):485-500.

Englmaier, F. and Fahn, M. (2019). Size Matters: How Over-Investments Relax Liquidity Constraints in Relational Contracts. The Economic Journal. uez029.

Englmaier, F. and Leider, S. (2012a). Contractual and organizational structure with reciprocal agents. American Economic Journal: Microeconomics, 4(2):146-183. 
Englmaier, F. and Leider, S. (2012b). Gift exchange in the lab - it is not (only) how much you give... CESifo Working Paper 2944.

Fahn, M. and Hakenes, H. (2019). Teamwork as a Self-Disciplining Device. American Economic Journal: Microeconomics.

Fahn, M., Schade, A., and Schuessler, K. (2017). What drives reciprocal behavior? the optimal provision of incentives over the course of careers. CESifo Working Paper No. 6635.

Fahn, M. and Zanarone, G. (2020). Accountability versus social comparisons: A theory of pay secrecy (and transparency) in organizations. Unpublished Working Paper.

Falk, A., Fehr, E., and Fischbacher, U. (2006). Testing theories of fairness - intentions matter. Games and Economic Behavior, 62(1):287-303.

Falk, A. and Fischbacher, U. (2006). A theory of reciprocity. Games and Economic Behavior, 54(2):293-315.

Fehr, E., Brown, M., and Zehnder, C. (2009). On reputation: A microfoundation of contract enforcement and price rigidity. The Economic Journal, 119(536):333-353.

Fehr, E. and Fischbacher, U. (2002). Why social preferences matter - the impact of nonselfish motives on competition, cooperation and incentives. The Economic Journal, 112(478):C1-C33.

Fehr, E., Kirchsteiger, G., and Riedl, A. (1993). Does fairness prevent market clearing? an experimental investigation. The Quarterly Journal of Economics, 108(2):437-459.

Fehr, E., Kirchsteiger, G., and Riedl, A. (1998). Gift exchange and reciprocity in competitive experimental markets. European Economic Review, 42(1):1-34.

Fehr, E. and Schmidt, K. M. (1999). A theory of fairness, competition, and cooperation. The Quarterly Journal of Economics, 114(3):817-868.

Fehr, E. and Schurtenberger, I. (2018). The dynamics of norm formation and norm decay. Working Paper.

Frederiksen, A., Lange, F., and Kriechel, B. (2017). Subjective performance evaluations and employee careers. Journal of Economic Behavior Organization, 134:408 - 429.

Gneezy, U. and List, J. A. (2006). Putting behavioral economics to work: Testing for gift exchange in labor markets using field experiments. Econometrica, 74(5):13651384. 
Haltiwanger, J. C., Lane, J. I., and Spletzer, J. R. (1999). Productivity differences across employers: The roles of employer size, age, and human capital. The American Economic Review, 89(2):94-98.

Harris, M. and Holmstrom, B. (1982). A theory of wage dynamics. The Review of Economic Studies, 49(3):315-333.

Hart, O. and Moore, J. (2008). Contracts as reference points. The Quarterly Journal of Economics, 123(1):1-48.

Hennig-Schmidt, H., Rockenbach, B., and Sadrieh, A. (2010). In search of workers' real effort reciprocity - a field and a laboratory experiment. Journal of the European Economic Association, 8(4):817-837.

Jayaraman, R., Ray, D., and de Véricourt, F. (2016). Anatomy of a contract change. American Economic Review, 106(2):316-58.

Kampkötter, P. and Sliwka, D. (2016). On the complementary use of experiments and field data to evaluate management practices - the case of subjective performance evaluations. Journal of Institutional and Theoretical Economics, 172(2):364-389.

Kessler, J. B. and Leider, S. (2012). Norms and contracting. Management Science, 58(1):62-77.

Kimbrough, E. O. and Vostroknutov, A. (2016). Norms make preferences social. Journal of the European Economic Association, 14(3):608 - 638.

Kragl, J. and Schmid, J. (2009). The impact of envy on relational employment contracts. Journal of Economic Behavior Organization, 72(2):766 - 779.

Krupka, E. L., Leider, S., and Jiang, M. (2017). A meeting of the minds: Informal agreements and social norms. Management Science, 63(6):1708-1729.

Lallemand, T. and Rycx, F. (2009). Are Young and Old Workers Harmful for Firm Productivity? Technical report.

Landmann, A. and Vollan, B. (2020). Pro-sociality of democratic leaders: Evidence from village elections in the philippines. Unpublished Working Paper.

Lazear, E. P., Shaw, K. L., and Stanton, C. (2016). Making do with less: Working harder during recessions. Journal of Labor Economics, 34(S1):S333-S360. 
Lemieux, T., MacLeod, W. B., and Parent, D. (2009). Performance Pay and Wage Inequality*. The Quarterly Journal of Economics, 124(1):1-49.

Levin, J. (2002). Multilateral contracting and the employment relationship. The Quarterly Journal of Economics, 117(3):1075-1103.

Levin, J. (2003). Relational incentive contracts. American Economic Review, 93(3):835-857.

MacLeod, W. B. and Malcomson, J. M. (1989). Implicit contracts, incentive compatibility, and involuntary unemployment. Econometrica, 57(2):447-480.

MacLeod, W. B., Valle Lara, V., and Zehnder, C. (2020). Worker empowerment and subjective evaluation: On building an effective conflict culture. Unpublished Working Paper.

Macneil, I. R. (1980). The New Social Contract. Yale University Press: New Haven.

Macneil, I. R. (1983). Values in contract: Internal and external. Northwestern University Law Review, 78:340-418.

Mailath, G. J. and Samuelson, L. (2006). Repeated games and reputations: long-run relationships. Oxford University Press.

Malcomson, J. M. (2013). Relational incentive contracts. In Gibbons, R. and Roberts, J., editors, The Handbook of Organizational Economics, pages 1014 - 1065. Princeton University Press.

Malmendier, U. and Schmidt, K. M. (2017). You owe me. American Economic Review, 107(2):493-526.

Mortensen, D. T. and Pissarides, C. A. (1999). Chapter 39 new developments in models of search in the labor market. volume 3 of Handbook of Labor Economics, pages 2567 - 2627. Elsevier.

Netzer, N. and Schmutzler, A. (2014). Explaining gift-exchange-the limits of good intentions. Journal of the European Economic Association, 12(6):1586-1616.

Prendergast, C. (1999). The provision of incentives in firms. Journal of Economic Literature, 37(1):7-63.

Rabin, M. (1993). Incorporating fairness into game theory and economics. The American Economic Review, 83(5):1281-1302. 
Ramey, G. and Watson, J. (1997). Contractual Fragility, Job Destruction, and Business Cycles. The Quarterly Journal of Economics, 112(3):873-911.

Reuben, E. and Suetens, S. (2012). Revisiting strategic versus non-strategic cooperation. Experimental Economics, 15(1):24-43.

Schmidt, K. M. (2011). Social preferences and competition. Journal of Money, Credit and Banking, 43(1):207-231.

Segal, U. and Sobel, J. (2007). Tit for tat: Foundations of preferences for reciprocity in strategic settings. Journal of Economic Theory, 136(1):197 - 216.

Shapiro, C. and Stiglitz, J. E. (1984). Equilibrium unemployment as a worker discipline device. The American Economic Review, 74(3):433-444.

Skirbekk, V. (2004). Age and individual productivity: A literature survey. Vienna Yearbook of Population Research, 2:133-153.

Sliwka, D. and Werner, P. (2017). Wage increases and the dynamics of reciprocity. Journal of Labor Economics, 35(2):299-344.

Sobel, J. (2005). Interdependent preferences and reciprocity. Journal of Economic Literature, 43(2):392-436. 